\title{
SOURCES OF INTERFERENCE IN ITEM AND ASSOCIATIVE RECOGNITION MEMORY: INSIGHTS FROM A HIERARCHICAL BAYESIAN ANALYSIS OF A GLOBAL MATCHING MODEL
}

\author{
DISSERTATION \\ Presented in Partial Fulfillment of the Requirements for the Degree Doctor of \\ Philosophy in the Graduate School of The Ohio State University \\ By \\ Adam F. Osth, B.A., M. A. \\ Graduate Program in Psychology
}

The Ohio State University

2014

Dissertation Committee:

Per Sederberg, Advisor

Roger Ratcliff

Jay Myung 
(C) Copyright by

Adam F. Osth

2014 


\section{Abstract}

A powerful theoretical framework for exploring recognition memory is the global matching framework, in which a cue's memory strength reflects the similarity of the retrieval cues being matched against all of the contents of memory simultaneously. Contributions at retrieval can be categorized as matches and mismatches to the item and context cues, including the self match (match on item and context), item noise (match on context, mismatch on item), context noise (match on item, mismatch on context), and background noise (mismatch on item and context). I present a model that directly parameterizes the matches and mismatches to the item and context cues, which enables estimation of the magnitude

of each interference contribution (item noise, context noise, and background noise). The model was fit within a hierarchical Bayesian framework to ten recognition memory datasets that employ manipulations of strength, list length, list strength, word frequency, studytest delay, and stimulus class in item and associative recognition. Estimates of the model parameters revealed at most a small contribution of item noise that varies by stimulus class, with virtually no item noise for single words and scenes. Despite the unpopularity of background noise in recognition memory models, background noise estimates dominated at retrieval across nearly all stimulus classes with the exception of high frequency words, which exhibited equivalent levels of context noise and background noise. These parameter estimates suggest that the majority of interference in recognition memory stems from experiences acquired prior to the learning episode. 
I would like to dedicate this dissertation to coffee. 


\section{ACKNOWLEDGMENTS}

I would like to acknowledge my former advisor, Simon Dennis, for the incredible help, influence, and encouragement on my work. I don't think I could have picked a better advisor.

The biggest motivation on my work has always been the stimulating conversations and tough questions I've received. For that reason, I would like to thank everybody that has generously donated their time to engaging conversations on the topic of memory research. A few names that come to mind include Troy Smith, Per Sederberg, Brandon Turner, Jeff Starns, Vishnu Sreekumar, and Greg Cox.

I would like to thank my parents for all the love and support they've shown me through all the challenges I've faced in the last six years. Even though they may never read this (or any of my other works), if it weren't for their support, this dissertation may never have gotten finished. 


\section{VITA}

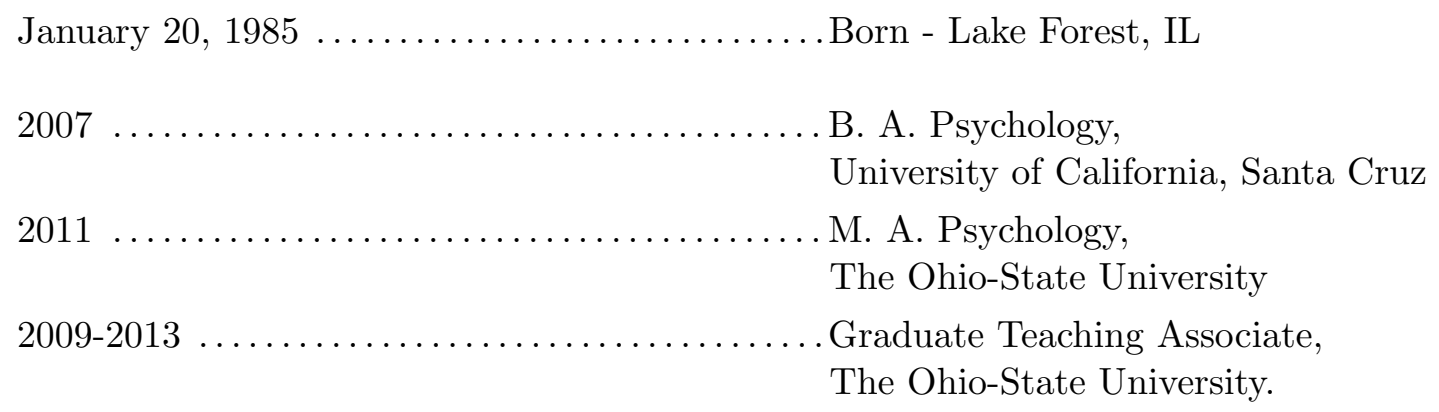

\section{Publications}

Osth, A. F., Dennis, S., \& Kinnell, A. (2014). Stimulus type and the list strength paradigm. The Quarterly Journal of Experimental Psychology. Online. Available at http://www .tandfonline.com/doi/abs/10.1080/17470218.2013.872824\#.UOSRNI7ztTM .

Osth, A. F., \& Dennis, S. (2013). Associative recognition and the list strength paradigm. Memory $\&$ Cognition. Online. Available at http://link.springer.com/article/10 .3758/s13421-013-0386-6.

Osth, A. F., \& Dennis, S. (2013). Constraints on theories of serial order memory revisited: The cases of the fill-in and protrusion effects. In M. Knauff, M. Pauen, N. Sebanz, \& I. Wachsmuth (Eds.), Proceedings of the XXXV Annual Conference of the Cognitive Science Society, (pp. 3199-3204). Austin, TX: Cognitive Science Society.

Osth, A. F., Dennis, S., \& Sloutsky, V. F. (2010). Context and category information in children and adults. In S. Ohlsson \& R. Catrambone (Eds.), Proceedings of the XXXII Annual Conference of the Cognitive Science Society, (pp. 842-847). Mahwah, NJ: Erlbaum.

Osth, A. F., \& Dennis, S. (2011). What are the boundary conditions of differentiation in episodic memory? In S. Ohlsson \& R. Catrambone (Eds.), Proceedings of the XXXIII Annual Conference of the Cognitive Science Society, (pp. 1230-1235). Mahwah, NJ: Erlbaum. 


\section{Fields of Study}

Major Field: Psychology

Studies in:

Development of Recognition Memory

Mathematical Modeling of Recognition Memory

Empirical Work on Memory for Serial Order

Simon Dennis \& Vladimir Sloutsky

Simon Dennis

Simon Dennis 


\section{Table of Contents}

Page

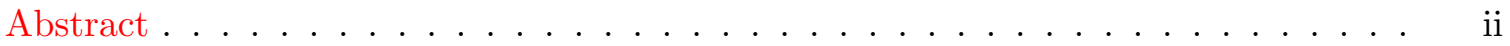

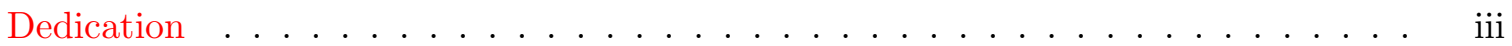

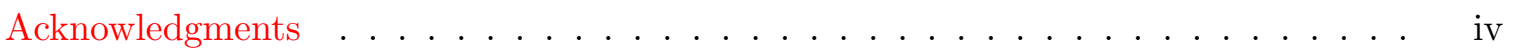

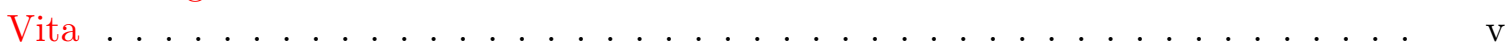

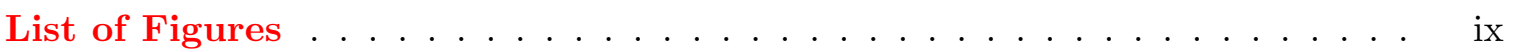

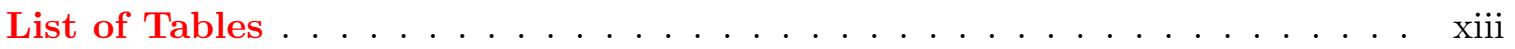

\section{Chapters}

1 Introduction $\quad \mathbf{1}$

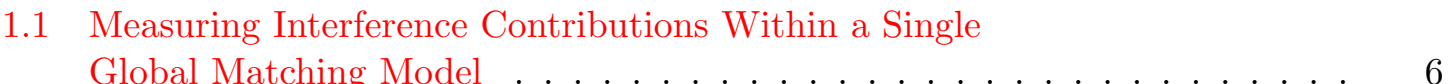

2 The Model $\quad 10$

2.1 The Context Mismatch Parameter, the Word Frequency Effect, and the Likelihood Ratio Transformation of Memory Strengths . . . . . . . . . . . . . . . 22

2.1.1 The Log Likelihood Ratio Transformation . . . . . . . . . . . . . 25

2.2 Unequal Variance Between the Target and Lure Distributions of Memory Strength . . . . . . . . . . . . . . . . . 27

2.3 The Item Mismatch Variability Parameter and Predictions of List Length and List Strength Effects . . . . . . . . . . . . . . . . . 30

2.4 Background Noise . . . . . . . . . . . . . . . . 34

3 The Model Fit $\quad 37$

3.1 Datasets Included in the Model Fit . . . . . . . . . . . . . . . . . . 38

3.1 .1 The ROC in item recognition . . . . . . . . . . . . . . 40

3.1.2 The List Length Paradigm . . . . . . . . . . . . . . . . . 41

3.1.3 The List Strength Paradigm . . . . . . . . . . . . . . . . . . . . . . 44

3.1 .4 Response Criteria . . . . . . . . . . . . . . . . . . 46

3.2 The Hierarchical Bayesian Model . . . . . . . . . . . . . . . . . 47

3.3 Analysis of the Model Fit . . . . . . . . . . . . . . . . . 50

3.4 Parameter Estimates . . . . . . . . . . . . . . . . . . . . . 51

3.5 Model Fit . . . . . . . . . . . . . . . . . . . 53

3.5.1 Word Frequency and Confidence Ratings: Fit to DeCarlo (2007) . . 54 
3.5.2 List Length, Word Frequency, and Study-Test Delay: Fit to Dennis,

Lee, and Kinnell (2008) . . . . . . . . . . . . . . 55

3.6 List Length and List Strength with Non-Linguistic Stimuli: Fit to Kinnell and Dennis (2012) and Osth et al. (2014) . . . . . . . . . 58

3.7 List Length and List Strength with Word Pairs in Associative Recognition: Fit to Kinnell and Dennis (2012) and Osth and Dennis (2013) . . . . . . . . 60

3.8 Interference Contributions . . . . . . . . . . . . . . . 66

4 General Discussion $\quad \mathbf{7 2}$

4.1 Plausibility of Low Item Noise . . . . . . . . . . . . . . . . 73

4.2 Arguments for Item Noise Models . . . . . . . . . . . . . . . . 75

4.2.1 Effects of Semantic Similarity on Recognition Memory . . . . . . . 76

4.2.2 Decrements in Performance Through Recognition Testing . . . . . . . 80

4.2.3 Evidence for Differentiation Models of Recognition Memory . . . . . 86

4.3 Differences Between Linguistic and Non-Linguistic Stimuli . . . . . . . . . . . 90

4.4 Other Sources of Variability in Recognition Memory . . . . . . . . . . . . . 92

4.5 Conclusion . . . . . . . . . . . . . . . . . . 93

$\begin{array}{ll}\text { References } & 95\end{array}$

\section{Appendices}

A Derivations for the Mean and Variance of Memory Strength in Associative Recognition

B Equations for the Log Likelihood Ratio Transformation of Memory Strength

C Binomial and Multinomial Rate Estimation 


\section{List of Figures}

Figure

Page

1.1 Diagram of the different sources of interference on retrieval within a global matching model. Depicted is a simplification the contents of memory, which include memories formed during the study list episode and memories from prior contexts. The probe cue and the study context are matched against the contents of memory simultaneously. The self match refers to an exact match on item and context information, in that the cues are matched against a representation of the cue item formed during the study list episode. Item noise refers to a match on context information but a mismatch on context information, in that the items are different from the probe cue but the memories were formed during the list episode. Context noise refers to a match on item information but a mismatch on context information, in that the memories are of the cue item but were formed prior to the list episode. Background noise refers to a mismatch on both item and context information. The magnitudes of each source of interference depend on the similarities between the matches and mismatches on item and context information. . . . . . . . . . . . .

2.1 Graph displaying how changes in the context mismatch variability can account for the word frequency mirror effect using both the raw memory strengths (top row) and after the log likelihood ratio transformation (bottom). In the left column are signal and noise distributions for low $(\rho=.025)$ and high $(\rho=.4)$ values of the context mismatch variability paramter, which correspond to low and high frequency words. In the middle column are predicted hit and false alarm rates for values of the context mismatch variability parameter ranging from .025 to 1.0. In the right column are the data from the 2AFC paradigm employed by Glanzer and Bowles (1976) along with model predictions (LF: $\rho=.05, \mathrm{HF}: \rho=.4$ ). All other model parameters are as follows: list length of 30 items, $r_{i t e m}=.4, \mu_{t t}=1, \mu_{s s}=1, \sigma_{t t}^{2}=.025$, $\sigma_{s s}^{2}=.075, \sigma_{t i}^{2}=.002, \beta_{i t e m}=.1 \ldots \ldots \ldots \ldots$ 
2.2 Graph displaying the effects of changes on the item match and context match variability parameters $\left(\sigma_{t t}^{2}\right.$ and $\left.\sigma_{s s}^{2}\right)$. Both parameters were initially set to .001. As both parameters are increased, the variability of the target distribution increases more than the variability of the lure distribution. Other parameters of the model were set as follows: list length $=20, r_{i t e m}=1$, $r_{\text {assoc }}=1, \mu_{t t}=1, \mu_{s s}=1, \sigma_{t i}^{2}=.002, \rho=.1, \beta_{\text {item }}=.15, \beta_{\text {assoc }}=.45 \ldots$.

2.3 Graph displaying demonstrations of the effects of the item mismatch variability parameter on both item recognition (top row) and associative recognition (bottom row), which controls the amount of item noise in the model. Depicted are simulations of a list length paradigm (left) and a list strength paradigm (middle column: HR and FAR predictions, right column: $d^{\prime}$ predictions). In the list length paradigm, the number of study list items was manipulated between 1 and 80 . In the list strength paradigm, 30 items were studied, half were baseline pairs studied with learning rate $r_{i t e m}=1.0$ and the other half were interference items studied with $r_{\text {item }}$ ranging between .05 and 2.5. The other parameters of the model were set as follows: $r_{\text {item }}=1$, $r_{\text {assoc }}=1, \mu_{t t}=1, \mu_{s s}=1, \sigma_{t t}^{2}=.025, \sigma_{s s}^{2}=.075, \rho=.1, \beta_{i t e m}=.1$, and

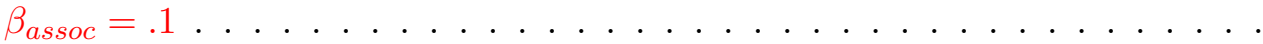

2.4 Graph displaying demonstrations of the effects of the item mismatch variability parameter $\left(\sigma_{t i}^{2}=0, .015, .03\right)$ on a mixed list of weak and strong pairs in associative recognition. Parameters were as follows: 15 weak pairs encoded with $r_{\text {assoc }}=1.0,15$ strong pairs encoded with $r_{\text {assoc }}=2.5, \mu_{t t}=1, \mu_{s s}=1$, $\sigma_{t t}^{2}=.025, \sigma_{s s}^{2}=.075$, and $\beta_{a s s o c}=.1 \ldots \ldots \ldots$

2.5 Graph displaying demonstrations of the effects of background noise both item recognition (top row) and associative recognition (bottom row). Background noise $\left(\beta_{\text {item }}\right.$ and $\left.\beta_{\text {assoc }}\right)$ was varied between $0, .4$, and 1.2. Depicted are simulations of a list length paradigm (left) and a list strength paradigm (middle column: HR and FAR predictions, right column: $d^{\prime}$ predictions). In the list length paradigm, the number of study list items was manipulated between 1 and 80 . In the list strength paradigm, 30 items were studied, half were baseline pairs studied with learning rate $r_{i t e m}=1.0$ and the other half were interference items studied with $r_{\text {item }}$ ranging between .05 and 2.5. The other parameters of the model were set as follows: $r_{i t e m}=1, \mu_{t t}=1, \mu_{s s}=1$, $\sigma_{t t}^{2}=.025, \sigma_{s s}^{2}=.075, \sigma_{t i}^{2}=.03, \rho=.001 \ldots \ldots \ldots \ldots$

3.1 Posterior distributions for all hyperparameters in the omnibus fit. Depicted parameters are the learning rate $r$ (learning rates for strong items/pairs are the sum of the weak and strong learning samples and are indicated by dashed lines), the mean context match $\mu_{s s}$, the variance in the item match $\sigma_{t t}^{2}$, the variance in the context match $\sigma_{s s}^{2}$, the item mismatch variance $\sigma_{t i}^{2}$, the context mismatch variance $\rho$, the background noise $\beta$, along with the decision criteria $\Phi$. Note: DLK = Dennis, Lee, and Kinnell (2008) dataset, DC = DeCarlo

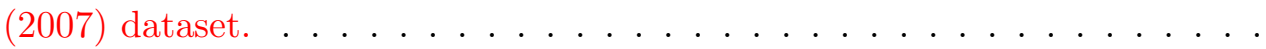

3.2 Density estimates of the differences between the means of the hyperparameters between words and other stimulus classes for both the item mismatch variability parameter $\sigma_{t i}^{2}$ (left) and the background noise parameter $\beta$ (right). 
3.3 Density estimates of the ROC function for both the data of DeCarlo (2007, left) and model (right) for low (top) and high (bottom) frequency words. The circles indicate the median of the hit and false alarm rate posterior distributions for each ROC point. . . . . . . . . . . . . . . .

3.4 Density estimates of the zROC function for both the data of DeCarlo (2007, left) and model (right) for low (top) and high (bottom) frequency words. The circles indicate the median of the z-transformed hit and false alarm rate posterior distributions for each ROC point. . . . . . . . . . . .

3.5 Individual participant responses for each confidence rating and word frequency class for the data of DeCarlo (2007). Predicted confidence counts from the model are shown as the colored density estimates. . . . . . . . . .

3.6 Fit to the data of Dennis et al. (2008) for all conditions. Depicted are the density estimates from group level parameters for both the data and the model, along with median posterior estimates for the data (circles) and the model (x's) . . . . . . . . . . . . . . . . . . . .

3.7 Individual participant hit and false alarm counts from the Dennis et al. (2008) dataset for all conditions. Predicted hit and false alarm counts from the model are shown as the colored density estimates. . . . . . . . . . .

3.8 Hit and false alarm rates for the data and the model fit to the list length experiments of Kinnell and Dennis (2012, left) and the list strength experiments of Osth et al. (2014, right). Depicted are the density estimates from group level parameters for both the data and the model, along with median posterior estimates for the data (circles) and the model (x's). . . . . . . .

3.9 Hit and false alarm rates for the data and the model fit to the list length experiments of Kinnell and Dennis (2012, left) and the list strength experiments of Osth et al. (2014, right) that employ non-linguistic stimuli. Depicted are the density estimates from group level parameters for both the data and the model, along with median posterior estimates for the data (circles) and the model (x's). . . . . . . . . . . . . . . . . . .

3.10 Individual participant hit and false alarm counts for the short (left) and long (right) list conditions for fractals (top), faces (middle), and scenes (bottom). Predicted hit and false alarm counts from the model are shown as the colored density estimates. . . . . . . . . . . . . . . . .

3.11 Individual participant hit and false alarm counts for the pure weak (left) and mixed weak (middle) and mixed strong (right) conditions for fractals (top), faces (middle), and scenes (bottom). Predicted hit and false alarm counts from the model are shown as the colored density estimates. False alarms are the same for the mixed weak and mixed strong conditions while the hits to 4X presented items are in the mixed strong plot. . . . . . . . . . . .

3.12 Hit and false alarm rates for the data and the model fit to the list length experiments of Kinnell and Dennis (2012, left) and the list strength experiments of Osth and Dennis (2013, right) that employ word pairs in an associative recognition task. Depicted are the density estimates from group level parameters for both the data and the model, along with median posterior estimates for the data (circles) and the model (x's) . . . . . . . . . . . . 
3.13 Hit and false alarm rates for the data and the model fit to the list length experiments of Kinnell and Dennis (2012, left) and the list strength experiments of Osth and Dennis (2013, right) that employ word pairs in an associative recognition task. Depicted are the density estimates from group level parameters for both the data and the model, along with median posterior estimates for the data (circles) and the model (x's). . . . . . . . . . . . .

3.14 Individual participant hit and false alarm counts for list length experiments of Kinnell and Dennis (2012, left) and the list strength experiments of Osth and Dennis (2013, right) that employ word pairs in an associative recognition task. Predicted hit and false alarm counts from the model are shown as the colored density estimates. For the mixed strong condition, hits and false alarm counts are from the strong intact and strong rearranged pairs. . . . .

3.15 Density estimates for item noise (IN), context noise (CN), and background noise $(\mathrm{BN})$ for all datasets. Notes: $\mathrm{LF}=$ low frequency, $\mathrm{HF}=$ high frequency, $\mathrm{SL}=$ short list, $\mathrm{LL}=$ long list, $\mathrm{PW}=$ pure weak, and $\mathrm{M}=$ mixed. . . . . .

3.16 Median proportions of total interference for item noise, context noise, and background noise (bg. noise) for the datasets of Dennis et al. (2008), Kinnell and Dennis (2012), Osth et al. (2014), and Osth and Dennis (2013). Error bars represent the $95 \%$ highest density interval (HDI). Notes: LF $=$ low frequency, $\mathrm{HF}=$ high frequency. . . . . . . . . . . . .

4.1 Group level predictions for the Schulman (1974) paradigm using parameters derived from the model fit, where on each individual trial two items are added to the contents of memory. The left panel shows the model predictions without parameter modification, while the right panel shows the model's predictions where each item on a test trial multiplies the mean context match by .9955 . Due to an inavailability of the original data from the Schulman (1974) dataset, the data means were approximated from the graphs in the original article. . . . . . . . . . . . . . . . .

4.2 Group data and model predictions for the Murdock and Anderson (1975) paradigm, where the number of choices on a forced choice recognition test was manipulated between two and six. The left panel shows the model predictions without parameter modification, while the right panel shows the model's predictions each item on a test trial multiplies the mean match to context by .9985. Note: The depicted data reflect averages across serial positions on the study list. . . . . . . . . . . . . . . . . . . . . 


\section{List of Tables}

Table

Page

2.1 Description of each of the model's parameters, including their boundaries and which conditions they change. . . . . . . . . . . . . . 21

3.1 Datasets included in the hierarchical Bayesian fit to the data. . . . . . . . . 39

3.2 All hyperparameters included in the hierarchical model. . . . . . . . . . . . 49 


\section{Chapter 1 INTRODUCTION}

Perhaps the biggest theoretical advance in recognition memory was the application of signal detection theory (SDT) by Egan (1958). SDT recast the role of a participant in a recognition memory experiment as having to decide between whether a presented stimulus is an instance of noise alone (a non-studied stimulus) or signal embedded in noise (a studied stimulus). This is accomplished by comparing the memory strength elicited by a stimulus (which is assumed to be continuously distributed) to a decision criterion on the memory strength axis; stimuli with memory strengths that exceed the decision criterion are judged as having occurred on the study list. Despite the utility of SDT in applications to measurement, it is agnostic as to the psychological content of the signal and noise distributions. Specifying the psychological content of the distributions requires process models that describe the encoding and retrieval operations of the memory system along with the content of the stored representations.

A watershed moment in process models of recognition memory came with the global matching models of recognition memory. While early theories of recognition memory described the signal and noise distributions as arising from a strength of the stimulus in memory (Wickelgren \& Norman, 1966), global matching models, following the encoding specificity principle of Tulving and Thomson (1973), posit that memory strength arises from the similarity between the retrieval cues and the contents of memory. Specifically, the cues are matched against all of the acquired memories in parallel, producing a single memory strength value that indexes the similarity of the cues to the contents of memory 
(Clark \& Gronlund, 1996; Humphreys, Pike, Bain, \& Tehan, 1989). In the majority of the global matching models, the distance between the signal and noise distributions arises from the match between the target item and its own representation in memory, whereas the variances of the two distributions arise primarily from spurious similarities between the cues and non-target representations stored in memory.

While several models have taken the simplifying assumption that only the memories from the study list contribute to the retrieval strengths, the frameworks are quite compatible with incorporating contributions from memories learned prior to an experiment. The division between recently acquired and prior memories can be reconciled quite easily by virtue of a context representation, which is now featured in the majority of episodic memory models (J. R. Anderson \& Bower, 1972; G. D. A. Brown, Preece, \& Hulme, 2000; Criss \& Shiffrin, 2004; Dennis \& Humphreys, 2001; Farrell, 2012; Gillund \& Shiffrin, 1984; Howard \& Kahana, 2002; Humphreys, Bain, \& Pike, 1989; Lehman \& Malmberg, 2013; Mensink \& Raaijmakers, 1988; Murdock, 1997; Shiffrin \& Steyvers, 1997). While there is no universally accepted definition of context, the central assumption among most theorists is that context is what enables retrieval to be focused on a particular episode, namely a study list (Klein, Shiffrin, \& Criss, 2007). Learning in contextual models does not merely consist of learning the stimulus, but instead consists of acquiring a binding of the stimulus and a representation of the current context into the contents of memory. At retrieval, the probe cue along with a reinstatement of the study context can be matched against the contents of memory. Under this view, memories of the list items can be distinguished from prior list memories by virtue of the similarity of the stored context representations to the context cues employed at retrieval. Specifically, successful discrimination relies on memories from the study list episode exhibiting more similarity to the context cue, whereas temporally distant memories should be relatively dissimilar to the current context to minimize interference.

The contributions from prior memories and current memories can be conceptualized as matches and mismatches of the stored memories to the item and context cues employed at retrieval, with the magnitudes of each interference contribution determined by the similarities of the matches and mismatches (see Figure 1.1). Specifically, the locus of successful 
discrimination is the self match, which is a match on stored item and context information to the item and context cues employed at retrieval. Other items from the study list episode match in context information, but mismatch in item information. An assumption adopted by the earliest global matching models, including Minerva 2 (Hintzman, 1988), the search of associative memory (SAM: Gillund \& Shiffrin, 1984) model, the theory of distributed associative memory (TODAM: Murdock, 1982), and the matrix model (Pike, 1984; Humphreys, Bain, \& Pike, 1989), is that the studied items of the list episode are the principal source of interference, an idea which has been retroactively referred to as the item noise conception of interference.

What was considered a strength of the pure item noise global matching models at the time was their ability to account for the list length effect in recognition memory performance, whereby performance decreases as the number of items on a list is increased (Strong, 1912). In global matching models, the spurious similarity between the retrieval cues and a stored memory produces a memory strength value with non-zero variance, and the variances of the resulting distributions are the sums of the variances of the individual matches. The list length effect naturally arises from the early global matching models because only the list items are assumed to be stored in memory and are therefore the principal source of interference. Thus, as more items are added to the contents of memory, the cumulative memory strength is a sum over a larger number of items and the variance in memory strengths for both targets and lures are increased, decreasing discriminability

An unintended consequence of pure item noise models is that the models predict a list strength effect in recognition memory performance. A list strength effect occurs when the strengthening of non-target items decreases performance on the target items. Global matching models predicted a list strength effect in recognition memory because repetitions of items had the same functional effect as increasing the length of a study list, as each repeated item in memory contributed additional variance to the retrieval process (for a complete description of how each global matching model was unable to predict a list strength effect, see Shiffrin, Ratcliff, \& Clark, 1990). However, Ratcliff et al.'s (1990) investigation found no effect of list strength on recognition memory performance, as the strenghtening 
a subset of list items did not impair recognition of the non-strengthened items and strong items did not benefit from being accompanied by weak items on a study list.

The null list strength effect was a strong constraint on the global matching models and various alternatives to the original global matching models were proposed. First, models with a revised encoding process called differentiation were proposed that reduce inter-item similarity as the strengths of the study items is increased, allowing for a reduction in item noise with increasing strength such that no detrimental effect of list strength is predicted. Additional items that are added to the contents of memory do not induce differentiation, and thus additional items increase the degree of item noise and a list length effect is predicted. Differentiation models include a modified version of the SAM model (Shiffrin et al., 1990), the retrieving effectively from memory model (REM: Shiffrin \& Steyvers, 1997) and the subjective likelihood in memory model (SLiM: McClelland \& Chappell, 1998, additional discussion on differentiation models can be found in the General Discussion). However, one of the main motivations behind the differentiation mechanism was to simultaneously predict a null list strength effect while predicting detrimental effects of increasing list length, and more recent evidence suggests that this dissociation may not be present.

Dennis and colleagues (Dennis \& Humphreys, 2001; Dennis, Lee, \& Kinnell, 2008; Kinnell \& Dennis, 2011) have noted that experiments that manipulate list length contain a number of confounds that may be causing worse performance in conditions with longer lists for reasons unrelated to interference among the list items. For instance, the retention intervals are shorter for short lists than long lists if testing immediately follows the end of the study list, reducing performance for items on the long list. When this confound and others are controlled, all experiments conducted by Dennis and colleagues have found no effect of list length on discriminability. Two other modifications to the global matching framework can allow for predicting null effects of list length and list strength on recognition memory performance.

Dennis and Humphreys (2001) argued that interference arises not from the list items (as assumed by pure item noise models) but from memories of the list items acquired prior to the experiment. Specifically, the retrieval cues are not just matched against representa- 
tions from the list episode, but are matched against all of the contexts in which the items were experienced to evaluate whether the context of the study list is included in the set of all stored context representations. This conception of interference has been referred to as context noise, because it is the past contexts in which the list items have been experienced (context mismatch) that generate interference in recognition memory (an idea which originated from J. R. Anderson \& Bower, 1972). Dennis and Humphreys (2001) introduced the bind-cue-decide model of episodic memory (BCDMEM), in which context noise was the sole source of interference in the model. Item representations in the model do not overlap with each other, meaning that the item mismatch penalty is zero and no effects of item noise, namely list length and list strength, are predicted. Dennis and Humphreys (2001) also demonstrated that the word frequency effect, in which words of low natural language frequency are better recognized than words of high natural language frequency (Glanzer \& Bowles, 1976; Glanzer \& Adams, 1985; Shepard, 1967), follows quite naturally from the concept of context noise: items that have been more frequently experienced have more associations to prior contexts, and thus there is more ambiguity as to whether or not they were seen in a given context. This is analogous to the way item noise models predict a list length effect in the sense that more stored representations produce additional interference at retrieval.

Another solution to the list strength effect came from Murdock and Kahana (Murdock, 1997; Murdock \& Kahana, 1993a, 1993b), who posited that global matching models should include a large number of memories that mismatch in both item and context information. This conception of interference has since been referred to as background noise by Osth, Dennis, and Kinnell (2014). Murdock and Kahana (1993a) argued that if the contribution of background noise is large relative to the item noise from the list items, then increases in interference that come from a list strength manipulation will produce only a negligible increase in the variances of the memory strength distributions, thus producing an approximate null list strength effect. Osth et al. (2014) revisited the idea of background noise for explaining the small item noise effects found for novel non-linguistic stimuli.

Kinnell and Dennis (2012) and Osth et al. (2014) conducted experiments using images of 
non-famous faces, random scenes, and generated fractals that are unlikely to have been ever witnessed by the participants prior to the experiment. Given the novelty of the stimuli, they cannot suffer from context noise as they have not been seen in prior contexts. Unlike words, small effects of list length (Kinnell \& Dennis, 2012) and list strength (Osth et al., 2014) were found for select non-linguistic stimuli employing the list length controls advocated by Dennis and colleagues. While effects of list length and list strength are not predicted by context noise models, they were much smaller than what would be predicted by pure item noise models. Osth et al. (2014) posited that novel non-linguistic stimuli might suffer from larger item noise than words, possibly due to exhibiting a higher degree of within-class inter-item similarity than words, but the effects of list length and list strength are somewhat mitigated by the additional influence of background noise at retrieval. Background noise has received relatively little attention in the recognition memory literature compared to the discussions of the influence of item and context noise. A diagram depicting the three sources of interference (item noise, context noise, and background noise) can be seen in Figure 1.1. The present investigation is focused on simultaneously measuring all three sources of interference by fitting a global matching model to a large number of recognition memory datasets.

\subsection{Measuring Interference Contributions Within a Single Global Matching Model}

A number of investigations have compared the relative merits of the item noise and context noise approaches to recognition memory using experimental data (Cho \& Neely, 2013; Criss, Malmberg, \& Shiffrin, 2011; Dennis \& Chapman, 2010; Dennis et al., 2008; Kinnell \& Dennis, 2011), with interpretations favoring either the item noise or context noise accounts, while there was little discussion of the role of background noise in any of these investigations. More recently, Turner, Dennis, and Van Zandt (2013) compared the REM (the original pure item noise version) and BCDMEM models in their ability to account for data from the list length paradigm (specifically the datasets of Dennis et al., 2008 and Kinnell \& Dennis, 2012) 


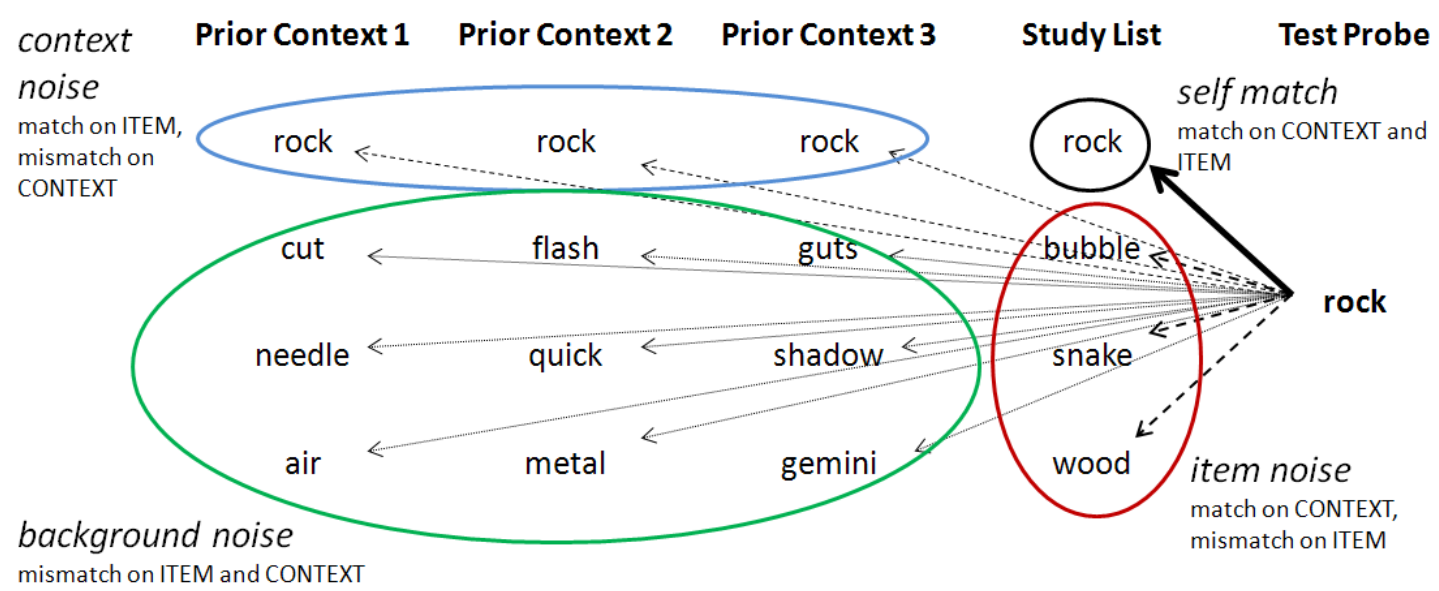

Figure 1.1: Diagram of the different sources of interference on retrieval within a global matching model. Depicted is a simplification the contents of memory, which include memories formed during the study list episode and memories from prior contexts. The probe cue and the study context are matched against the contents of memory simultaneously. The self match refers to an exact match on item and context information, in that the cues are matched against a representation of the cue item formed during the study list episode. Item noise refers to a match on context information but a mismatch on context information, in that the items are different from the probe cue but the memories were formed during the list episode. Context noise refers to a match on item information but a mismatch on context information, in that the memories are of the cue item but were formed prior to the list episode. Background noise refers to a mismatch on both item and context information. The magnitudes of each source of interference depend on the similarities between the matches and mismatches on item and context information. 
using hierarchical Bayesian methods. BCDMEM consistently exhibited lower values of the deviance information criterion (DIC), a Bayesian model selection measure that measures goodness of fit relative to the degree of model complexity. Turner et al. (2013) attributed the superior performance of the BCDMEM model to the fact that the null list length effect in the data is a compulsory prediction of the BCDMEM model, whereas REM exhibits flexibility in its predicted magnitude of the list length effect. The higher DIC value assigned to the REM model may indicate that this flexibility is an unwarranted complexity of the model, and the authors attributed this strength to a parsimony of the context noise account.

However, as noted by Criss and Shiffrin (2004), item noise and context noise are not mutually exclusive; it is completely plausible for a memory system to suffer from both item noise and context noise at retrieval. Not only does there remain the underexplored interference contribution of background noise, but there is also the possibility that the magnitude of each interference contribution depends on the stimulus class being employed. I present the results of fitting a global matching model to a large number of recognition memory datasets and measuring the respective contributions of item noise, context noise, and background noise to the total interference contribution at retrieval. The model is a variation on the tensor model presented by Humphreys, Bain, and Pike (1989) in which memory is a composite of three-way bindings between two items and the experimental context. Unlike the original tensor model, the item and context vectors are not specified. Instead, the parameters of the model are the similarities between the item and context vectors. This property makes the model well suited to measuring the three sources of interference, as the parameters of the model directly specify the strengths associated with the item match and context match along with the penalties incurred by the item mismatch and context mismatch, each of which can be used to calculate the magnitude of the item noise, context noise, and background noise contributions. The datasets included in the fit include manipulations of all of the variables that are required to constrain the parameters of the model, such as strength, word frequency, list length, list strength, and study-test delay.

Furthermore, a critical limitation of the BCDMEM model was its inability to account 
for stimuli other than single words (which have the necessary background experience to suffer from context noise) and that it lacks a mechanism for inter-item binding. As I have mentioned previously, experiments with novel non-linguistic stimulus classes uncovered small detrimental effects of list length and list strength, which are consistent with item noise models but inconsistent with context noise models (Kinnell \& Dennis, 2012; Osth et al., 2014). The model I present is capable of addressing these sources of interference and I have included the experiments conducted in these two papers to compare the interference contributions across the different stimulus classes.

Additionally, I have included experiments conducted using the associative recognition task, in which participants study a list of pairs (such as A-B, C-D, E-F, etc.) and are asked to discriminate between studied pairs (such as A-B, referred to as intact pairs) and studied words presented in a novel arrangement (such as C-F, referred to as rearranged pairs). There has been relatively little discussion as to the sources of interference in the associative recognition task in the literature. I include the results of two experiments, one that manipulated list length (Kinnell \& Dennis, 2012) and one that manipulated list strength (Osth \& Dennis, 2013) to measure the sources of interference in associative recognition.

The outline for the remainder of the paper is as follows. First, I describe the variation of the Humphreys, Bain, and Pike (1989) model and how it calculates the three sources of interference that have been postulated to affect recognition memory. I also discuss a necessary addition to the model to address the mirror effects present in the data, namely the log likelihood ratio transformation of memory strengths by Glanzer, Hilford, and Maloney (2009). Next, I give a summary of the ten datasets that were used in the model fitting along with a description of how the parameters used in the fitting matched the experimental manipulations. I then describe how the models were fit using hierarchical Bayesian methods to get simultaneous estimates of both subject and group level parameters. I then present the results of the model fitting procedure along with several analyses of the resulting subject and group level parameters to compare the respective contributions of the sources of interference. 


\section{Chapter 2 The Model}

I follow the tradition of several memory models and represent both items and contexts as vectors of features. To simplify description as much as possible, I define items as single stimuli that are presented to the participant and the context as a representation that defines the list episode. I follow several other episodic memory models in my assumption that item features and context features are independent of each other (G. D. A. Brown et al., 2000; Criss \& Shiffrin, 2004; Dennis \& Humphreys, 2001; Humphreys, Bain, \& Pike, 1989; Mensink \& Raaijmakers, 1988; Murdock, 1997; Shiffrin \& Steyvers, 1997) . Bindings between items and contexts are represented as outer products of the constituent item and context vectors. Psychologically, outer products reflect conjoint representation of the constituents that bears little similarity to any of the constituent vectors (similar to the convolution operation; Murdock, 1985; Pike, 1984).

Evidence supporting conjoint representations comes from a study by Dosher and Rosedale (1989) in which participants studied triplets of items and were tested on pairs from the triplets in an associative recognition task. Successful priming was only found when the entire triplet was completed by the prime, such as if a triplet ABC was studied and item A preceded the pair BC. Dosher and Rosedale (1989) found no evidence for priming on partial matches, such as item A preceding a BF pair trial. Similarly, Hockley and Cristi (1996b) conducted a judgment-of-frequency (JOF) task in which both items and pairs were studied

\footnotetext{
${ }^{1}$ Another possible assumption that is employed by the temporal context model (Howard \& Kahana, 2002) is that context features are the previously encountered items, causing a high correlation between item features and context features. However, models that employ this assumption of context have yet to be applied comprehensively to data from recognition memory paradigms.
} 
and participants made JOFs on both studied items and pairs. Some of the items that were presented alone were constituents of the pairs, e.g.: item A presented alone, and also as part of pair A-B. Hockley and Cristi (1996b) found that the frequency of A had no influence on the judged frequency of $\mathrm{A}-\mathrm{B}$, as if the $\mathrm{A}$ representations and $\mathrm{A}-\mathrm{B}$ representations did not overlap with each other.

When a list of items is studied, the model stores outer products of the item and the list context on each trial. These outer products are then summed together to produce an occurrence matrix $M_{i}$ :

$$
M_{i}=\sum_{a} r_{i t e m} C_{s} \otimes I_{a}
$$

where $C$ denotes a context vector, $I$ denotes an item vector, and the subscript $s$ refers to the fact that the context vector represents the study episode. To account for variation in strength of learning, due either to different rates of presentation, different numbers of presentations, or differences among participants in their ability to encode the material, I use a scalar $r_{\text {item }}$ that is applied to the outer products as a learning rate parameter.

When a list of pairs are studied for an associative recognition task, the model stores three-way outer products between the two items in the pair and the list context as a mode three tensor product. These tensor products are then summed together to produce the co-occurrence tensor $M_{c}$ :

$$
M_{o}=\sum_{a, b} r_{a s s o c} C_{s} \otimes I_{a} \otimes I_{b}
$$

where $r_{a s s o c}$ is the learning rate for associative information. I would like to emphasize that I employ a separate tensor representation for associative recognition purely for mathematical convenience and are not committed to the idea that the occurrence matrix and co-occurrence tensor reflect different neurological substrates or stores. I also make the simplifying assumption that when participants are studying word pairs in an associative recognition task the only inter-item associations that are formed are among the pair members (a similar assumption was made by Gillund \& Shiffrin, 1984). I have also alotted a separate learning 
rate parameter for associative information based on the finding that encoding manipulations produce different effects on item and associative recognition. For instance, Hockley and Cristi (1996a) found that deep encoding manipulations that emphasize item information enhance item recognition but punish associative recognition, whereas deep encoding manipulations that emphasize associative information enhance both item and associative recognition. Thus, the model allows for the possibility that encoding strength can be strong in both item and associative recognition, weak in one task but not the other, etc.

Memory strength is computed by combining the cues available at retrieval into an outer product and matching it against the appropriate memory store. In the case of item recognition, this involves construcing an outer product of the probe cue and the context cue employed at retrieval. This matrix is matched against the occurrence matrix $M_{i}$ :

$$
s=\left(C_{s}^{\prime} \otimes I_{a}^{\prime}\right) \cdot M_{i}
$$

where $s$ is a scalar that represents the memory strength generated from the global match of the cues against the contents of memory. The dashes on the context and item vectors are used to indicate that the item vector representing the probe and the context vector representing the context at the time of test may not perfectly resemble the vectors that were used at the time of study. A cue for a target item may not resemble the vector that was originally stored due to variation in perceptual processing of the stimulus (McClelland \& Chappell, 1998). The context at test may either be an imperfect reinstatement of the study context (G. D. A. Brown et al., 2000; Dennis \& Humphreys, 2001) or a context that has drifted from the original stored study context as a consequence of the events that have intervened between study and test (Howard \& Kahana, 2002; Mensink \& Raaijmakers, 1988; Murdock, 1997).

The equation is the same for associative recognition, except it involves combining both item cues with the context vector into a tensor representation and matching it against the 
co-occurrence tensor $M_{c}$. The equation is as follows:

$$
s=\left(C_{s}^{\prime} \otimes I_{a}^{\prime} \otimes I_{b}^{\prime}\right) \cdot M_{c}
$$

It is common at this stage for the vectors to be generated from sampling distributions with a finite number of elements. The number of elements in a vector can be considered a parameter of the model despite the fact that it contains no obvious psychological interpretation. When this parameter is free to vary in a model fit, the model parameters are no longer able to be identified in models such as REM (Montenegro, Myung, \& Pitt, 2011) and BCDMEM (Myung, Montenegro, \& Pitt, 2007). As a consequence, it is common practice for the vector size parameter in a model to be fixed to an arbitrary value.

I instead use an approximate analytic solution that specifies the similarities between the vectors without specifying the content of the vectors themselves. Such an approach is convenient as it allows the different sources of interference in retrieval to be parameterized as matches and mismatches among the context and item vectors. The analytic solution is obtained by decomposing the retrieval equation into each of the component matches in a manner similar to that used by Humphreys, Pike, et al. (1989) in their analyses of the global matching models.

For the case of item recognition, Equation 2.3 can be rewritten by decomposing the occurrence matrix $M_{i}$ into matches among all of the stored memories. If the probe item is a target item, the equation is as follows:

$$
\begin{aligned}
& s=\left(C_{s}^{\prime} \otimes I_{t}^{\prime}\right) \cdot r_{i t e m}\left(C_{s} \otimes I_{t}\right) \quad \text { Self Match } \\
& +\sum_{i \in L, i \neq t} r_{i t e m}\left(C_{s} \otimes I_{i}\right) \quad \text { Item Noise } \\
& +\sum_{u \in P, u \neq s}\left(C_{u} \otimes I_{t}\right) \quad \text { Context Noise } \\
& +\sum_{u \in P, u \neq s, z \notin L}\left(C_{u} \otimes I_{z}\right) \quad \text { Background Noise }
\end{aligned}
$$

The first term in the right column is the original studied item in the study list context. The match between this matrix and the matrix cue can be referred to as the self match 
(match to both item and context cues) which is not present in the matching equation for a lure. The self match determines the difference in the means between the signal and noise distributions.

The second term in the right column is all of the study list items that are not the target item. The $L$ subscript refers to the set of all of the list items. Similarity between the cue item $I_{t}^{\prime}$ and the stored list items produces item noise. As was previously mentioned, the majority of the original global matching models tended to only consider self matches and item noise and never considered the role of pre-experimental interference. Nonetheless, interference from pre-experimentally stored memories could be expected to play a role in memory retrieval, and I consider their possible matches below.

The third term in the right column is the match of the probe item to all of its preexperimentally stored representations. The $u$ subscript of the context vector denotes that these stored contexts are different from the study list context and the $U$ subscript in the sum refers to the set of all contexts over a lifetime that are not the study list context. Similarity among the reinstated context cue $C_{s}^{\prime}$ and the pre-experimental contexts produces context noise at retrieval. The BCDMEM model can be considered an example of a global matching model that only considers the self matches and context noise at retrieval.

The fourth term in the right column is the match of the probe item to everything else that has been stored in memory. That is, all memories that mismatch in both item and context information are contained in this term. If these memories overlap with the matrix cue they would produce interference that I refer to as background noise. As I have mentioned previously, this term does not contribute in most memory models, with the exceptions of the TODAM (Murdock \& Kahana, 1993a, 1993b) and TODAM2 (Murdock, 1997) models.

Equation 2.5 can be rewritten as the match between the test cues and the stored vectors 
in memory:

$$
\begin{array}{rr}
s= & \text { Self Match } \\
\left.\sum_{i \in L, i \neq t} r_{i t e m}\left(C_{s}^{\prime} \cdot C_{s}\right)\left(I_{t}^{\prime} \cdot I_{t}\right)+C_{s}\right)\left(I_{t}^{\prime} \cdot I_{i}\right)+ & \text { Item Noise } \\
\sum_{u \in P, u \neq s}\left(C_{s}^{\prime} \cdot C_{u}\right)\left(I_{t}^{\prime} \cdot I_{t}\right)+ & \text { Context Noise } \\
\sum_{u \in P, u \neq s}\left(C_{s}^{\prime} \cdot C_{u}\right)\left(I_{t}^{\prime} \cdot I_{i}\right) & \text { Background Noise }
\end{array}
$$

The three sources of interference (item noise, context noise, and background noise) are now described as matches and mismatches between the item and context vectors. These dot products can be parameterized using normal distributions:

$$
\begin{array}{rlr}
C_{s}^{\prime} . C_{s} & \sim \operatorname{Normal}\left(\mu_{s s}, \sigma_{s s}^{2}\right) & \text { Context Match } \\
C_{s}^{\prime} \cdot C_{u} & \sim \operatorname{Normal}\left(\mu_{s u}, \sigma_{s u}^{2}\right) & \text { Context Mismatch } \\
I_{t}^{\prime} \cdot I_{t} & \sim \operatorname{Normal}\left(\mu_{t t}, \sigma_{t t}^{2}\right) & \text { Item Match } \\
I_{t}^{\prime} \cdot I_{i} & \sim \operatorname{Normal}\left(\mu_{t i}, \sigma_{t i}^{2}\right) & \text { Item Mismatch }
\end{array}
$$

The means and variances of the distributions of dot products are the parameters of the model. While this approach is somewhat unorthodox, it is similar to the kernel trick employed by support vector machines (Schölkopf \& Smola, 2002).

The matches in Equation 2.7 include the match between the test context and the context of study (context match, indexed by subscript ss), the match between the test context and contexts prior to the study list (context mismatch, subscript $s u$ ), the match between the probe cue and its own stored item representation (item match, subscript $t t$ ), and the match between the probe cue and other items stored in memory (item mismatch, subscript ti). The distributions of the matches and mismatches can be substituted into the terms for Equation 2.6 to derive mean and variance expressions for the signal and noise distributions. Because each interference term is the multiplication of an item match/mismatch by a context match/mismatch, and each are represented by normal distributions, each term is 
a multiplication of normal distributions which results in a modified Bessel function of the third kind with mean and variance as follows:

$$
\begin{aligned}
& E\left(X_{1} X_{2}\right)=\mu_{1} \mu_{2} \\
& V\left(X_{1} X_{2}\right)=\mu_{1}^{2} \sigma_{2}^{2}+\mu_{2}^{2} \sigma_{1}^{2}+\sigma_{1}^{2} \sigma_{2}^{2}
\end{aligned}
$$

However, given the large number of list items and non-list items that are stored in the occurrence matrix, the final distribution of memory strength is the sum of many product distributions and the sum is approximately normal by virtue of the central limit theorem. The mean and variance for the old and new distributions are as follows:

$$
\begin{aligned}
\mu_{\text {old }} & =r_{i t e m} \mu_{s s} \mu_{t t}+r_{i t e m}(l-1) \mu_{s s} \mu_{t i}+m \mu_{s u} \mu_{t t} \\
\mu_{\text {new }} & =r_{i t e m} l \mu_{s s} \mu_{t i}+m \mu_{\text {su }} \mu_{t t}
\end{aligned}
$$

$$
\begin{array}{cr}
\sigma_{\text {old }}^{2}=r_{i t e m}^{2}\left(\mu_{s s}^{2} \sigma_{t t}^{2}+\mu_{t t}^{2} \sigma_{s s}^{2}+\sigma_{s s}^{2} \sigma_{t t}^{2}\right)+ & \text { Self Match } \\
r_{i t e m}^{2}(l-1)\left(\mu_{s s}^{2} \sigma_{t i}^{2}+\mu_{t i}^{2} \sigma_{s s}^{2}+\sigma_{s s}^{2} \sigma_{t i}^{2}\right)+ & \text { Item Noise } \\
m\left(\mu_{s u}^{2} \sigma_{t t}^{2}+\mu_{t t}^{2} \sigma_{s u}^{2}+\sigma_{s u}^{2} \sigma_{t t}^{2}\right)+ & \text { Context Noise } \\
n\left(\mu_{s u}^{2} \sigma_{t i}^{2}+\mu_{t i}^{2} \sigma_{s u}^{2}+\sigma_{s u}^{2} \sigma_{t i}^{2}\right) & \text { Background Noise } \\
& \\
\sigma_{\text {new }}^{2}=r_{i t e m}^{2} l\left(\mu_{s s}^{2} \sigma_{t i}^{2}+\mu_{t i}^{2} \sigma_{s s}^{2}+\sigma_{s s}^{2} \sigma_{t i}^{2}\right)+ & \text { Item Noise } \\
m\left(\mu_{s u}^{2} \sigma_{t t}^{2}+\mu_{t t}^{2} \sigma_{s u}^{2}+\sigma_{s u}^{2} \sigma_{t t}^{2}\right)+ & \text { Context Noise } \\
n\left(\mu_{s u}^{2} \sigma_{t i}^{2}+\mu_{t i}^{2} \sigma_{s u}^{2}+\sigma_{s u}^{2} \sigma_{t i}^{2}\right) & \text { Background Noise }
\end{array}
$$

where $l$ is the length of the list, $m$ is the number of pre-experimental memories of the target item, and $n$ is the total number of background memories. The rows of Equation 2.10 can be viewed as the contributions of the self match, item noise, context noise, and background noise. Equations 2.10 and 2.11 are identical with the exception of the self match variance 
term which is only in Equation 2.10 and the fact that item noise is scaled by $l-1$ in Equation 2.11 instead of $l$. These equations also reveal the various effects of item noise, context noise, and background noise. Increases in the number of memories, such as the number of list memories $l$, prior occurrences of the cue $m$, and other stored memories $n$ all increase the variance of the old and new distributions in a linear fashion.

Some simplicitations are made in order to reduce the number of parameters and avoid covariances. Specifically, in Equation 2.6, one can see that there are multiple item matches and context matches across the different interference terms. To avoid covariances between these matches, I fix the parameters $\mu_{t i}$ and $\mu_{s u}$ to zero, a simplication which also has the effect of fixing the mean of the lure distribution at zero. There is precedent for such an approach, as both the Minerva 2 (Hintzman, 1988) and TODAM (Murdock, 1982) models have a lure distribution that is fixed at zero by usage of zero-centered vectors, which ensures that the expected match between any two vectors is zero.

In addition, I am more interested in the variance contribution in the context noise and background noise term than I am in identifying the number of stored memories. For that reason, I ignore the $m$ term and instead allocate separate context mismatch variability parameters to high and low frequency items to reflect the varying degrees of context noise. I denote the combined influence of $m$ and $\sigma_{s u}^{2}$ as parameter $\rho$. Additionally, I eliminate the entire background noise term and instead substitute a separate variance parameter to reflect its contribution, which I denote as $\beta$. The simplified equations are as follows:

$$
\begin{aligned}
\mu_{\text {old }} & =r_{i t e m} \mu_{s s} \mu_{t t} \\
\mu_{\text {new }} & =0
\end{aligned}
$$




$$
\begin{array}{cr}
\sigma_{\text {old }}^{2}=r_{i t e m}^{2}\left(\mu_{s s}^{2} \sigma_{t t}^{2}+\mu_{t t}^{2} \sigma_{s s}^{2}+\sigma_{s s}^{2} \sigma_{t t}^{2}\right)+ & \text { Self Match } \\
r_{i t e m}^{2}(l-1)\left(\mu_{s s}^{2} \sigma_{t i}^{2}+\sigma_{s s}^{2} \sigma_{t i}^{2}\right)+ & \text { Item Noise } \\
\left(\mu_{t t}^{2} \rho+\rho \sigma_{t t}^{2}\right)+ & \text { Context Noise } \\
\beta & \text { Background Noise } \\
\sigma_{\text {new }}^{2}=r_{i t e m}^{2} l\left(\mu_{s s}^{2} \sigma_{t i}^{2}+\sigma_{s s}^{2} \sigma_{t i}^{2}\right)+ & \text { Item Noise } \\
\left(\mu_{t t}^{2} \rho+\rho \sigma_{t t}^{2}\right)+ & \text { Context Noise } \\
\beta & \text { Background Noise }
\end{array}
$$

Each interference term in Equations 2.14 and 2.15 arises from combinations of the matches and mismatches of context and item information. The mean of the target distribution is a multiplication of the learning rate $r_{i t e m}$, the mean of the item match $\mu_{t t}$, and the mean of the context match $\mu_{s s}$. In my fits of the model to data, I fixed the mean of the item match $\mu_{t t}$ to one for simplicity ${ }^{2}$. I vary the mean context match $\mu_{s s}$ across conditions that vary in retention interval to reflect the loss of study context information from contextual drift or imperfect reinstatement of the study context. For conditions where testing is either immediate or follows shortly after the study list, I fix the value of $\mu_{s s}$ at one.

All mismatch parameters contribute to the variances of the distributions rather than the means. The self match variability is a function of the mean and variances of the item and context matches (as will be seen below, appropriate choices of these parameter values can instill higher variance in the target distribution than the lure distribution). The item noise term is a function of the number of list items multiplied by the variability in the item mismatch $\sigma_{t i}^{2}$, which is scaled by both of the context match parameters. The context noise term is a function of the variability in the context mismatch $\rho$ scaled by both of the item

\footnotetext{
${ }^{2}$ It would be plausible for the mean of the item match to vary across conditions that vary in stimulus strength. It is extremely plausible that the different stimulus classes in this investigation vary in item strength, but was simpler to assume that all of the differences arose from differences in learnability.
} 
match parameters.

Both the self match and item noise terms are scaled by the learning rate. Thus, as the encoding strength is increased, both the self match variability and the item noise are increased. The increase in item noise is the locus of the list strength effect. In the list strength datasets considered here, two list conditions are used: one where all items are presented once (the pure weak condition) and one where half the items are presented four times and half the items are presented once (the mixed condition). Specification of mixed list interference requires learning rates for weak and strong items along with separate item noise terms for each learning rate that are added together. A list strength effect would be present if performance on the once presented weak items is worse in the mixed list relative to the pure weak list, and arises if the item noise from the strong items is sufficiently larger than the item noise from the weak items. As I will later demonstrate, a null list strength effect can be predicted if either a.) item noise is sufficiently low from a low value of the item mismatch parameter $\sigma_{t i}^{2}$ or b.) the ratio of background noise to item noise is sufficiently high such that the strong item interference presents only a negligible addition to the total interference.

To avoid redundancy in the text, derivations of the distributions for associative recognition can be found in Appendix A. The same distributions for the item and context matches and mismatches are used. The mean and variances of the resulting memory strength distributions for intact and rearranged pairs are as follows:

$$
\begin{aligned}
\mu_{\text {int. }} & =r_{\text {assoc }} \mu_{s s} \mu_{t t}^{2} \\
\mu_{\text {rearr. } .} & =0
\end{aligned}
$$




$$
\begin{aligned}
& \sigma_{\text {int. }}^{2}=r_{\text {assoc }}^{2}\left(2 \mu_{s s}^{2} \mu_{t t}^{2} \sigma_{t t}^{2}+\mu_{s s}^{2} \sigma_{t t}^{4}+\sigma_{s s}^{2} \mu_{t t}^{4}+2 \sigma_{s s}^{2} \mu_{t t}^{2} \sigma_{t t}^{2}+\sigma_{s s}^{2} \sigma_{t t}^{4}\right)+\quad \text { Self Match } \\
& r_{a s s o c}^{2}(l-1)\left(\mu_{s s}^{2} \sigma_{t i}^{4}+\sigma_{s s}^{2} \sigma_{t i}^{4}\right) \\
& \beta_{\text {assoc }} \\
& \sigma_{\text {rearr. }}^{2}=2 r_{\text {assoc }}^{2}\left(2 \mu_{s s}^{2} \mu_{t t}^{2} \sigma_{t i}^{2}+\mu_{s s}^{2} \mu_{t t}^{2} \sigma_{t i}^{2}+\sigma_{s s}^{2} \mu_{t t}^{2} \sigma_{t i}^{2}+\sigma_{s s}^{2} \sigma_{t t}^{2} \sigma_{t i}^{2}\right)+ \\
& r_{\text {assoc }}^{2}(l-2)\left(\mu_{s s}^{2} \sigma_{t i}^{4}+\sigma_{s s}^{2} \sigma_{t i}^{4}\right)+ \\
& \beta_{\text {assoc }} \\
& \text { Item Noise } \\
& \text { Background Noise }
\end{aligned}
$$

The interference terms are very similar to the derivations for item recognition with a few exceptions. The first is that there is no context noise term; this is because the associative recognition task involves random pairings of unrelated words and thus the probability of having seen a given pair multiple times prior to the experiment is negligible (this assumption would not apply if previously associated pairs are employed). Second, there is an additional partial match term for the rearranged pairs in Equation 2.19. This reflects the fact that for a rearranged pair A-D, there are two partially matching pairs in memory: A-B and C-D. Additionally, given that there are likely to be many more combinations of item-itemcontext bindings than single item-context bindings, a separate background noise term was alotted for the co-occurrence tensor $\left(\beta_{\text {assoc }}\right)$. All of the model parameters along with their descriptions can be found in Table 2.1. Next, I describe the likelihood ratio transformation of memory strengths that is necessary to capture the full range of mirror effects seen in recognition memory data. 
Table 2.1: Description of each of the model's parameters, including their boundaries and which conditions they change.

\begin{tabular}{|c|c|c|}
\hline Param & Bounds & Description \\
\hline $\begin{array}{l}r_{\text {item }} \\
r_{\text {assoc }}\end{array}$ & $0: 1$ & $\begin{array}{l}\text { Learning rates for items and associations. Increase with } \\
\text { study time or repetitions. }\end{array}$ \\
\hline$\mu_{s s}$ & $0: 1$ & $\begin{array}{l}\text { Context match mean: matching strength of test context cue } \\
\text { to stored context. Contributes to the mean of the target } \\
\text { distribution. Decreases with study-test delay. }\end{array}$ \\
\hline$\sigma_{t t}^{2}$ & $0: \infty$ & $\begin{array}{l}\text { Item match variability: Variability of the match of the item } \\
\text { cue to the stored item. Increases the variability of the target } \\
\text { distribution relative to the lure distribution. }\end{array}$ \\
\hline$\sigma_{s s}^{2}$ & $0: \infty$ & $\begin{array}{l}\text { Context match variability: Variability of the match of the } \\
\text { context cue to the stored context. Increases the variability } \\
\text { of the target distribution relative to the lure distribution. }\end{array}$ \\
\hline$\rho$ & $0: \infty$ & $\begin{array}{l}\text { Context mismatch variability: Contributes to the amount } \\
\text { of context noise in the model. Expected to vary with word } \\
\text { frequency and is zero for items not seen in prior contexts. }\end{array}$ \\
\hline$\sigma_{t i}^{2}$ & $0: \infty$ & $\begin{array}{l}\text { Item mismatch variability: Contributes to the amount of } \\
\text { item noise in the model. Magnifies effects of list length and } \\
\text { list strength manipulations. Varies by stimulus class. }\end{array}$ \\
\hline $\begin{array}{l}\beta_{\text {item }} \\
\beta_{\text {assoc }}\end{array}$ & $0: \infty$ & $\begin{array}{l}\text { Background noise for items and associations. Obscures ef- } \\
\text { fects of list length/list strength on performance. Varies by } \\
\text { stimulus class. }\end{array}$ \\
\hline$\Phi$ & $-\infty: \infty$ & Response criterion. 0 represents an unbiased criterion. \\
\hline
\end{tabular}




\subsection{The Context Mismatch Parameter, the Word Frequency Effect, and the Likelihood Ratio Transformation of Mem- ory Strengths}

A strong constraint on models of recognition memory is the word frequency mirror effect, in which low frequency (LF) words have higher hit rates and lower false alarm rates than high frequency $(\mathrm{HF})$ words ${ }^{3}$. The mirror effect was described as a challenge to simple strength models of recognition memory (Glanzer \& Adams, 1985, 1990). As I will demonstrate, this is partially true. The basic pattern of the mirror effect can be achieved using the memory strength computation in the model, but there is evidence from two alternative forced choice (2AFC) testing that suggests a mirror ordering in the means of the distributions, which requires a likelihood ratio transformation of memory strengths.

The locus of the word frequency effect in the model is the context mismatch variability parameter $\rho$, which primarily contributes to the context noise term in Equations 2.14 and 2.15 for item recognition. From inspection of the equations, one can see that context noise is produced by a multiplication of the item match parameters $\mu_{t t}$ and $\sigma_{t t}^{2}$ along with the context mismatch variability parameter $\rho$. Critically, when context mismatch variability is zero, there is no context noise. This reflects the idea that there is no similarity between the current context and the previously stored contexts: the current context cue $C_{s}^{\prime}$ is perfectly able to isolate the list items from pre-experimentally stored memories. This is an implicit assumption in the early global matching models that assumed that only the list items contributed to interference at retrieval.

When context mismatch variability is greater than zero, greater interference arises from items that are more frequently represented in memory. As a consequence, high frequency words suffer more interference than low frequency words. Model predictions with different values of the context mismatch parameter $\rho$ can be seen in the middle panel of Figure 2.1. It is interesting to note that the model is able to predict a mirror effect of word frequency: as frequency increases, hit rates decrease and false alarm rates increase. This is because

\footnotetext{
${ }^{3} \mathrm{~A}$ mirror effect refers to any manipulation that exerts opposite effects on the hit rates and false alarm rates (Glanzer \& Adams, 1985)
} 
context noise, like item noise, is a variance term for both target and lure items. For a fixed decision criterion, an increase in variance of both distributions will cause a mirror effect.

Glanzer and Bowles (1976) conducted a thorough test of the locus of the word frequency effect using $2 \mathrm{AFC}$ tests. In a $2 \mathrm{AFC}$ tests, it is assumed that a response criterion for a stimulus is not used. Instead, the choice is selected that is furthest on the decision axis (T. D. Wickens, 2002). Glanzer and Bowles (1976) manipulated the composition of the choices on 2AFC tests, using all possible combinations of old and new items such as LFold and LF-new trials (LO-LN), LF-old and HF-new trials (LO-HN), HF-old and LF-new trials (HO-LN), and HF-old and HF-new trials (HO-HN). The mirror effect was obtained in all cases, and the ordering of the probability of correct choice was as follows: LO-LN > $\mathrm{LO}-\mathrm{HN} \approx \mathrm{HO}-\mathrm{LN}>\mathrm{HO}-\mathrm{HN}$. While the occurrence of the mirror effect in 2AFC testing is challenging to criterion shift accounts of the mirror effect (e.g.: Gillund \& Shiffrin, 1984), inspection of the top right panel of Figure 2.1 reveals that the variance account in the model is capable of addressing this pattern (LO-LN $>$ LO-HN $\approx$ HO-LN $>$ HO-HN).

However, there two more trial types that the variance account is unable to address. Both of these trial types can be considered null comparisons because they are not valid trials with one correct choice and one incorrect choice. In both types of trials, one word is $\mathrm{LF}$ and the other is $\mathrm{HF}$, but in one there are two targets (LO-HO) and in another there are two lures (HN-LN). Surprisingly, in the target trials, the LF word is chosen more often $(p(\mathrm{LO}, \mathrm{HO})>.5)$ but in the lure trials, the HF word is chosen more often $(p(\mathrm{HN}$, $\mathrm{LN})>.5)$. One can see that in Figure 2.1, the variances account fails to produce choice probabilities that are greater than .5 for the null comparison trials. Glanzer and Bowles (1976) noted that what is necessary is a mirror ordering arrangement of the means of the signal and noise distributions, such as $\mathrm{LN}<\mathrm{HN}<\mathrm{HO}<\mathrm{LO}$. Mirror arrangements of the underlying distributions can be produced using log likelihood ratio transformation of the memory strengths. 


\section{Word Frequency Effects}
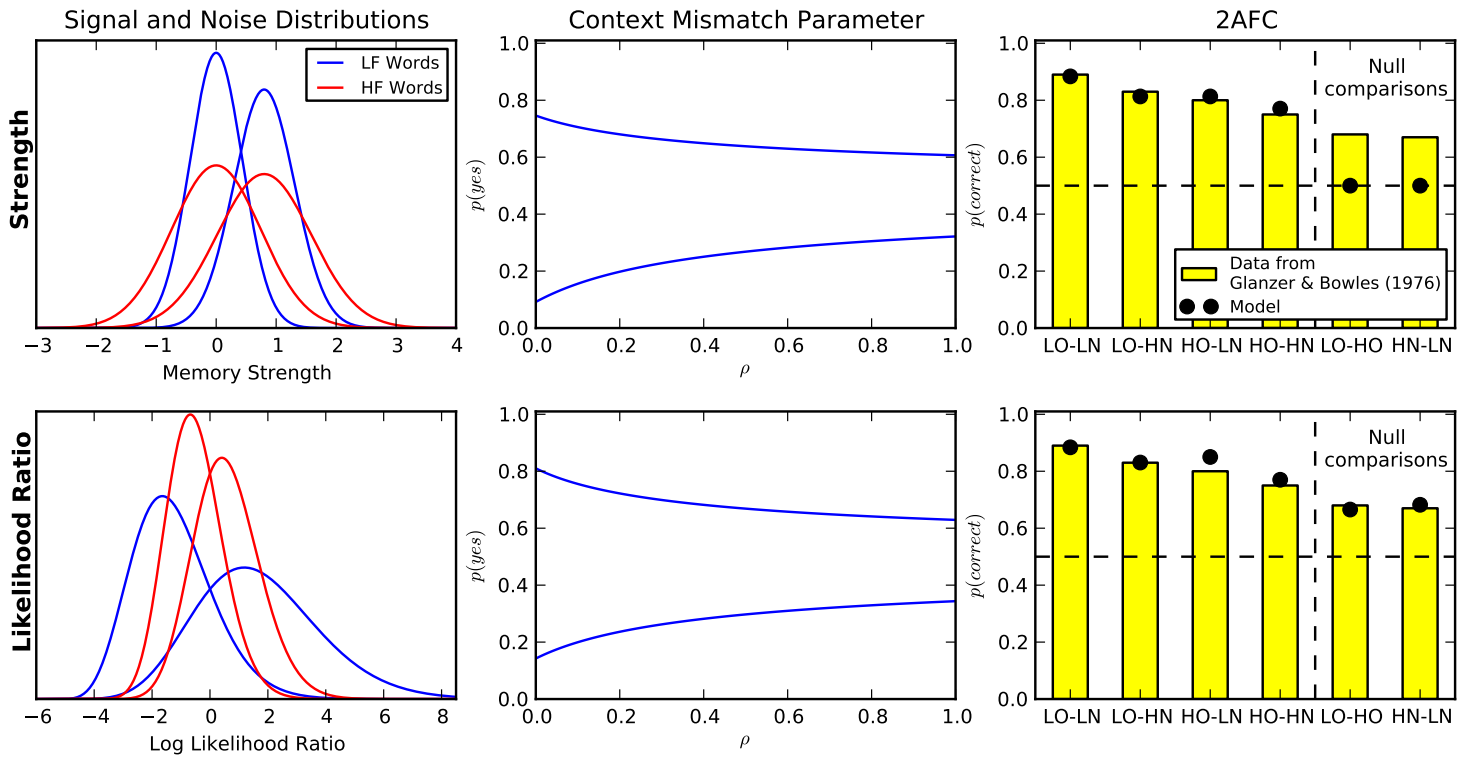

Figure 2.1: Graph displaying how changes in the context mismatch variability can account for the word frequency mirror effect using both the raw memory strengths (top row) and after the log likelihood ratio transformation (bottom). In the left column are signal and noise distributions for low $(\rho=.025)$ and high $(\rho=.4)$ values of the context mismatch variability paramter, which correspond to low and high frequency words. In the middle column are predicted hit and false alarm rates for values of the context mismatch variability parameter ranging from .025 to 1.0. In the right column are the data from the $2 \mathrm{AFC}$ paradigm employed by Glanzer and Bowles (1976) along with model predictions (LF: $\rho=.05$, HF: $\rho=.4$ ). All other model parameters are as follows: list length of 30 items, $r_{\text {item }}=.4$, $\mu_{t t}=1, \mu_{s s}=1, \sigma_{t t}^{2}=.025, \sigma_{s s}^{2}=.075, \sigma_{t i}^{2}=.002, \beta_{\text {item }}=.1$. 


\subsubsection{The Log Likelihood Ratio Transformation}

Several current models of recognition memory employ likelihood ratios as the basis of a recognition memory decision to produce the mirror effect (Dennis \& Humphreys, 2001; Glanzer \& Adams, 1990; Glanzer, Adams, Iverson, \& Kim, 1993; McClelland \& Chappell, 1998; Shiffrin \& Steyvers, 1997). In log likelihood ratio models, decisions are not made on the basis of memory strength, but are instead made on the basis of a ratio of the densities of the signal and noise distributions. To understand how the mirror effect is derived from a likelihood ratio, take a point on the $\mathrm{x}$ axis of the signal and noise distributions in the top left panel of Figure 2.1. Compare the relative heights of the targets and lures of the HF distributions at that point: that ratio is the likelihood ratio for the stimulus that elicited that value of memory strength. On the same point on the $\mathrm{x}$ axis, consider the LF distributions. In the case of targets, the density of the target distribution greatly exceeds the density of the lure distribution, producing a higher likelihood ratio. Note that for targets, the density greatly exceeds the density of the lure distribution due to the lower overlap of the distributions, producing higher likelihood ratios for LF words and increasing the hit rate. The opposite is the case for lures (greater lure-to-target density for LF words, producing lower likelihood ratios). A simple psychological interpretation of the likelihood ratio transformation is that memory strength is not considered alone, but is instead considered along with the knowledge about the memorability of the stimlus (similar to the account proposed by J. Brown, Lewis, \& Monk, 1977). I will demonstrate that the expected memorabilities need not correspond perfectly to the actual memory strength distributions.

The mirror effect has been demonstrated to be a regularity of the likelihood ratio transformation (Glanzer et al., 1993, 2009). There are other advantages to the likelihood ratio transformation, such as the concentering of the likelihood ratio distributions in response to manipulations that decrease performance (Glanzer, Adams, \& Iverson, 1991; Hilford, Glanzer, \& Kim, 1997; Kim \& Glanzer, 1993, 1995), shorter zROC lengths for conditions of stronger performance (Stretch \& Wixted, 1998a; Glanzer et al., 2009), as well as higher 
variances for distributions that are further from the criterion (as measured by old-old and new-new zROC slopes: DeCarlo, 2007; Glanzer et al., 2009).

I have employed analytic solutions for the log likelihood ratio transformation that were developed by Glanzer et al. (2009) to be used with unequal variance normal signal detection models. After the transformation has been applied, it results in log likelihood ratio distributions that are non-central chisquare in shape. I have had to modify the equations to consider cases in which the model has access to incomplete information about the study episode. For instance, consider a case in which items were either studied once (weak) or four times (strong). During the test phase, participants are tested on weak and strong targets in addition to lures. This leads to three memory strength distributions: one for lures, one for weak targets, and one for strong targets. If it's assumed that during weak target trials participants calculate the likelihood ratio using the weak target distribution as reference, the model has already presupposed memory of the test stimulus. Instead, under conditions in which mixed lists of items are studied, the expected strength in the likelihood ratio calculation is a distribution that reflects the average of the learning rate parameters corresponding to the weak and strong items ${ }^{4}$. Expected strengths were used in the likelihood calculations in the BCDMEM model (Dennis \& Humphreys, 2001; Starns, White, \& Ratcliff, 2010). The modified equation for the likelihood ratio transformation can be found in Appendix B. For all other parameter variations outside of the learning rate differences in mixed strength study lists, the expected parameter values are identical to the actual parameter values of the memory strength distributions.

Predictions of the log likelihood ratio transformation can be seen in the bottom row of Figure 2.1. The left panel reveals that the transformation correctly produces the mirror ordering of the distributions $(\mathrm{LN}>\mathrm{HN}>\mathrm{HO}>\mathrm{LO})$. The model produces patterns that are quite similar to the variance account, but inspection of the $2 \mathrm{AFC}$ predictions in the right panel of Figure 2.1 reveals that the model is correctly able to predict null comparisons

\footnotetext{
${ }^{4}$ Another case where expected strengths would be employed is for modeling the effects of serial position. Items from different serial positions have different study-test lags, suggesting different values of the mean context match $\mu_{s s}$. The expected context match at test could be constructed from the average of the context match parameters for each serial position.
} 
that are quite close to the experimental data $(p(\mathrm{LO}, \mathrm{HO})$ and $p(\mathrm{HN}, \mathrm{LN})>.5)$. The $\log$ likelihood ratio transformation is applied to all model predictions from this point on in the article.

\subsection{Unequal Variance Between the Target and Lure Distri- butions of Memory Strength}

The slope of the z-transformed ROC is almost uniformly less than one in the recognition memory literature (Egan, 1958; Glanzer, Kim, Hilford, \& Adams, 1999; Heathcote, 2003; Ratcliff, Sheu, \& Gronlund, 1992; Ratcliff, McKoon, \& Tindall, 1994). Within an SDT model, the common interpretation is the variability of the target distribution is greater than the lure distribution. When the distributions are normal in shape, the slope is the ratio of standard deviations $\sigma_{\text {new }} / \sigma_{\text {old }}$.

How can unequal variance be produced by the model? The variances of the target and lure distributions in item recognition are nearly identical except that for the case of targets, there are $l-1$ items in the item noise term and an additional self match term. If the self match variability exceeds the item noise for a single item (before being scaled by l), then targets will exhibit higher variability than lures. The model can accomplish this with sufficient values of either the item match variability $\left(\sigma_{t t}^{2}\right)$ or context match variability $\left(\sigma_{s s}^{2}\right)$. The effects of these variables can be seen in Figure 2.2 for both item recognition (top) and associative recognition (bottom): both the item match variability parameter $\sigma_{t t}^{2}$

and context match variability parameter $\sigma_{s s}^{2}$ were set to .02 and separately incremented to higher values. As the values of these parameters are increased, one can see that the ratio of standard deviations increases.

Why do item and context match variabilities produce unequal variance between the target and lure distributions? One might be inclined to think that the target item was seen in the list context, so variation in how the item was processed or variation in the match of the test context to the stored context should affect targets but not lures. However, both of these factors also affect the degree of match to previously stored memories. Inspection of 

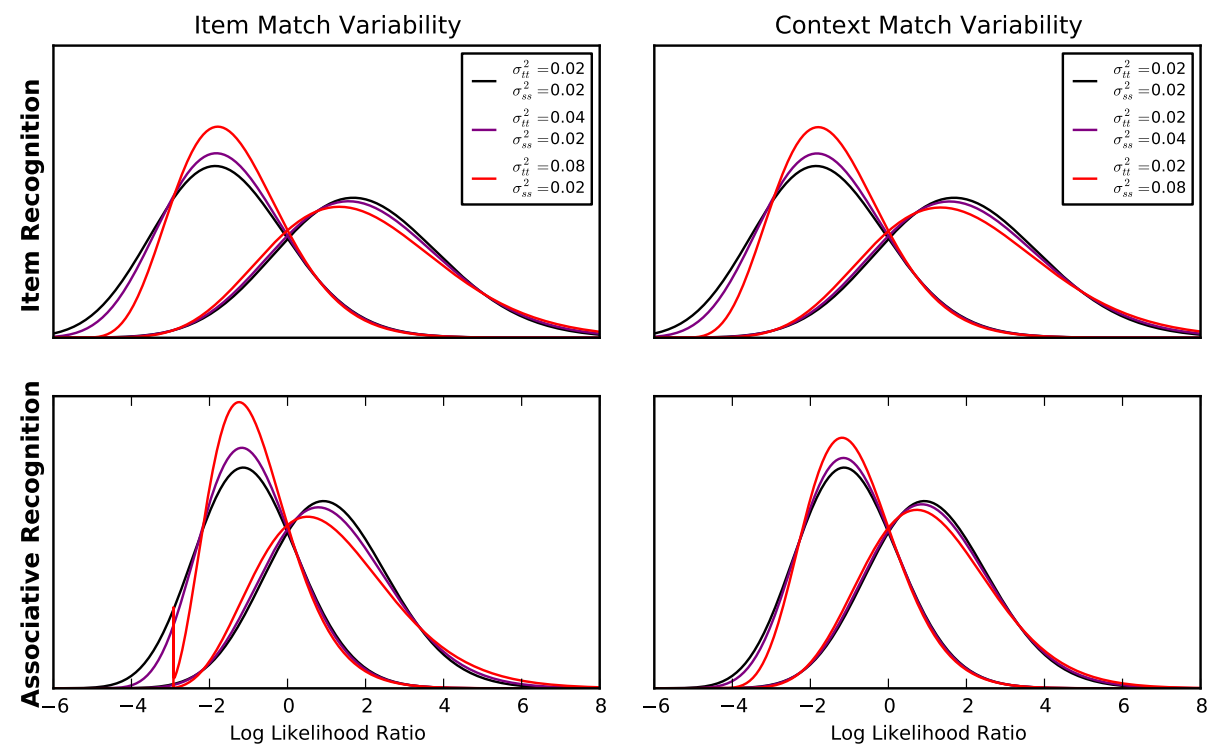

Figure 2.2: Graph displaying the effects of changes on the item match and context match variability parameters $\left(\sigma_{t t}^{2}\right.$ and $\left.\sigma_{s s}^{2}\right)$. Both parameters were initially set to .001 . As both parameters are increased, the variability of the target distribution increases more than the variability of the lure distribution. Other parameters of the model were set as follows: list length $=20, r_{\text {item }}=1, r_{\text {assoc }}=1, \mu_{t t}=1, \mu_{s s}=1, \sigma_{t i}^{2}=.002, \rho=.1, \beta_{\text {item }}=.15$, $\beta_{\text {assoc }}=.45$. 
Equations 2.14 and 2.15 reveals that the item mismatch in the item noise term is scaled by the context match and the context mismatch in the context noise term is scaled by the item match. Unequal variance is produced because in the self match term of Equation 2.14, there are more non-zero means contributing to the variance calculation (the learning rate $r$, the item match $\mu_{t t}$, and the context match $\mu_{s s}$ ). Thus, the simple answer is that the variability of the target distribution increases naturally with its mean, however the magnitude of this increase is modulated by the item and context match variability parameters. If the item and context match variability parameters exceed the item and context mismatch variability parameters, unequal variance that resembles the magnitude found in recognition memory experiments can be produced. As the item and context matching strengths decrease to zero, the ratio of standard deviations should approach 1. Ratcliff et al. (1994) found that with very low study times, the slope of the zROC is very close to 1 . I constrain the values that these two parameters take by including ROC data, namely the dataset of DeCarlo (2007), in the model fit.

Much of the discussion about the source of unequal variance in recognition memory has focused on the hypothesis of Wixted (2007) that unequal variance arises due to variability in the strength of learning. That is, some items on the study list may be encoded with more strength than others, producing an additional source of variability for target items. The variability in learning strength hypothesis has been tested recently with mixed results (Koen \& Yonelinas, 2010, 2013; Starns, Rotello, \& Ratcliff, 2012; Jang, Mickes, \& Wixted, 2012). I would like to note that variability in the item match (which could arise from factors such as variability in the perceptual processing of the probe item) along with variability in the context match (which could arise from noise in either the contextual reinstatement process or the contextual drift process) are plausible contenders for sources of unequal variance that have not received attention in the literature. I do not mean this to imply that variability in the strength of learning is not responsible for unequal variance, but merely that unequal variance may reflect variability in several processes employed at both encoding and retrieval. 


\subsection{The Item Mismatch Variability Parameter and Predic- tions of List Length and List Strength Effects}

As mentioned in the introduction, global matching models that only consider the role of item noise at retrieval predict detrimental effects of list length and list strength on recognition memory performance. From inspection of the item noise terms for item recognition (Equations 2.14 and 2.15) and associative recognition (Equations 2.18 and 2.19), one can see that item noise is produced by a multiplication of the item mismatch variability parameter $\sigma_{t i}^{2}$, the context mismatch variability parameter $\sigma_{s s}^{2}$, the learning rate $r$, and the number of items or pairs on the list $l$. The most critical of these parameters is the item mismatch variability. If this is set to zero, the entire item noise term is zero and no effect of list length or list strength is predicted. For positive values of the item mismatch variability parameter, increases in the number of list items $l$ or the learning rate $r$ increase the total item noise variance, which are the list length and list strength predictions, respectively.

These predictions can be seen in Figure 2.3 for both item recognition (top) and associative recognition (bottom). Depicted are two demonstrations for three different values of the item mismatch variability parameter $\sigma_{t i}^{2}: 0, .02$, and .04. The first demonstration is of a list length manipulation in which list length is varied between 1 and 80 items or pairs. Item noise increases linearly with increases in the list length for positive values of item mismatch variability. Consequently, performance decreases rapidly with increases in list length. When there is no item mismatch variability, item noise is zero and no effect of list length is predicted.

The list strength paradigm was simulated by using a 30 item list in item recognition and 30 pairs in associative recognition. Half of the items are baseline items that were studied with learning rate $r=1.0$. The other half are interference items and were studied with learning rates varying between .05 and 2.5 and performance was assessed on the baseline items. As mentioned previously, for mixed lists of strong and weak items, the likelihood ratio computation compares items to a mixed distribution of strong and weak items. Thus, as the strength of the interference items is increased, the strength of the mixed distribution 
increases. This increase in the expected memorability of test items decreases the hit rates and false alarm rates of the non-strengthened items. This allows the model to predict the strength based mirror effect in item and associative recognition, which is where strengthening a set of items increases the hit rate of the strengthened items and simultaneously decreases the false alarm rate (Hirshman, 1995; Hockley \& Niewiadomski, 2007; Stretch \& Wixted, 1998b).

Due to the decrease in hit rates and false alarm rates that occur with increases in strength, the list strength effect can be more easily observed by observing how $d^{\prime}$ changes as the strength of the interference items and pairs is increased. When item mismatch variability is zero, there is no item noise and the strength of the interference items/pairs has no impact on performance. For positive values of item mismatch variability, performance degrades quickly as the strength of the interference items and pairs is increased.

One may also note that effects of both list length and list strength are smaller in associative recognition than in item recognition. That is because associative recognition involves the multiplication of two items instead of one, meaning that the item mismatch variability parameter $\sigma_{t i}^{2}$ is squared. When the values are less than one, $\sigma_{t i}^{2}$ 's influence on item noise will be smaller for item recognition than for associative recognition. However, it is not the case that the item mismatch variability parameter has no visible effect on associative recognition predictions.

A number of researchers have conducted investigations using mixed lists of strong and weak pairs in associative recognition. On the test lists, rearranged pairs that came from both strong and weak pairs were presented and the false alarm rates were compared. A majority of investigations have found no difference between weak and strong rearranged pairs (e.g.: Buchler, Light, \& Reder, 2008; Kelley \& Wixted, 2001). As initially noted by Osth and Dennis (2013), the degree to which false alarm rates increase with strength in a mixed list is a consequence of item noise. To understand why, consider the partial match term in the equation for $\sigma_{\text {rearr. }}^{2}$ :

$$
2 r_{a s s o c}^{2}\left(2 \mu_{s s}^{2} \mu_{t t}^{2} \sigma_{t i}^{2}+\mu_{s s}^{2} \mu_{t t}^{2} \sigma_{t i}^{2}+\sigma_{s s}^{2} \mu_{t t}^{2} \sigma_{t i}^{2}+\sigma_{s s}^{2} \sigma_{t t}^{2} \sigma_{t i}^{2}\right)
$$




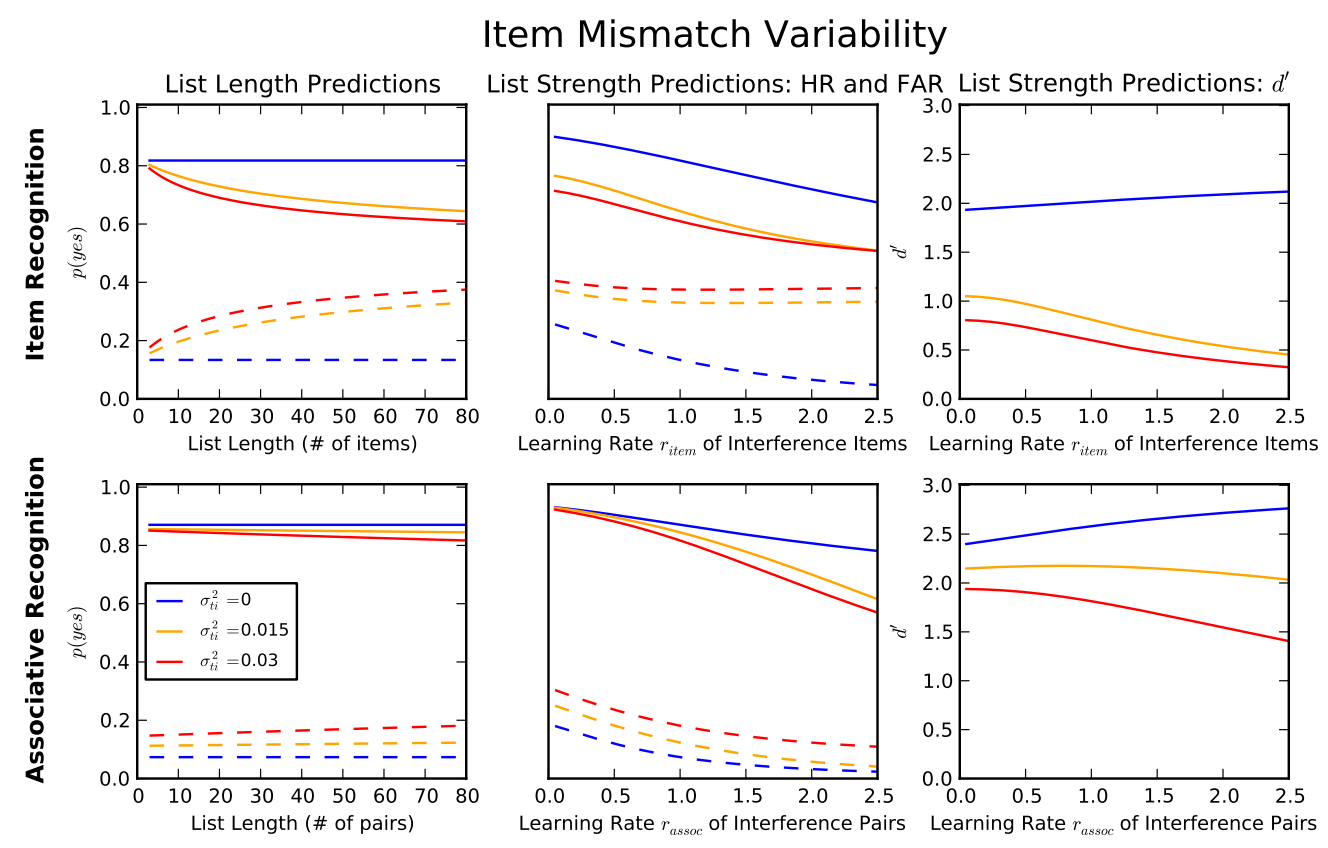

Figure 2.3: Graph displaying demonstrations of the effects of the item mismatch variability parameter on both item recognition (top row) and associative recognition (bottom row), which controls the amount of item noise in the model. Depicted are simulations of a list length paradigm (left) and a list strength paradigm (middle column: HR and FAR predictions, right column: $d^{\prime}$ predictions). In the list length paradigm, the number of study list items was manipulated between 1 and 80. In the list strength paradigm, 30 items were studied, half were baseline pairs studied with learning rate $r_{i t e m}=1.0$ and the other half were interference items studied with $r_{\text {item }}$ ranging between .05 and 2.5. The other parameters of the model were set as follows: $r_{i t e m}=1, r_{\text {assoc }}=1, \mu_{t t}=1, \mu_{s s}=1$, $\sigma_{t t}^{2}=.025, \sigma_{s s}^{2}=.075, \rho=.1, \beta_{\text {item }}=.1$, and $\beta_{\text {assoc }}=.1$ 
When item mismatch variability is zero, the interference from the two partial matches A-B and C-D to a rearranged pair A-D reduces to zero. When it is positive, the partial match term scales by the learning rate $r_{a s s o c}$, meaning that the two stored pairs exert greater interference on a rearranged pair cue as their strength is increased. This means that for a mixed list of strong and weak pairs, strong rearranged pairs suffer greater partial match interference when item mismatch variability is high.

The effect of item mismatch variability on mixed lists of strong and weak pairs can be seen in Figure 2.4. Three different levels of the parameter were compared $\left(\sigma_{t i}^{2}=0, .015, .03\right)$ for a mixed list with 15 weak pairs studied with a learning rate $r_{a s s o c}=1.0$ and 15 strong pairs were studied with $r_{a s s o c}=2.5$. When item mismatch variability is zero, false alarm rates are equivalent between weak and strong rearranged pairs. However, as the item mismatch variability is increased, false alarm rates are considerably higher for strong rearranged pairs than for weak rearranged pairs.

While null effects of list strength (e.g.: Ratcliff, Clark, \& Shiffrin, 1990) and list length (e.g.: Dennis et al., 2008) are commonly found with word stimuli, novel non-linguistic stimuli such as fractal and face images have been found to be susceptible to effects of both list length (Kinnell \& Dennis, 2012) and list strength (Osth et al., 2014; Norman, Tepe, Nyhus, \& Curran, 2008). As I will demonstrate in the fits to the datasets, this can be accommodated by alotting separate item mismatch parameters to each stimulus class to reflect the idea that the item representations of each stimulus class may vary in their degree of inter-item similarity. Modeling the complete interference contributions between all the stimulus classes would require a matrix of item mismatch variability parameters that reflects item similarity within a given stimulus class and between the different stimulus classes. However, given that in all of the datasets included in the model fit only test one stimulus class, I simplify treatment by only considering within-class interference. 


\section{Associative Recognition: Mixed List Predictions}
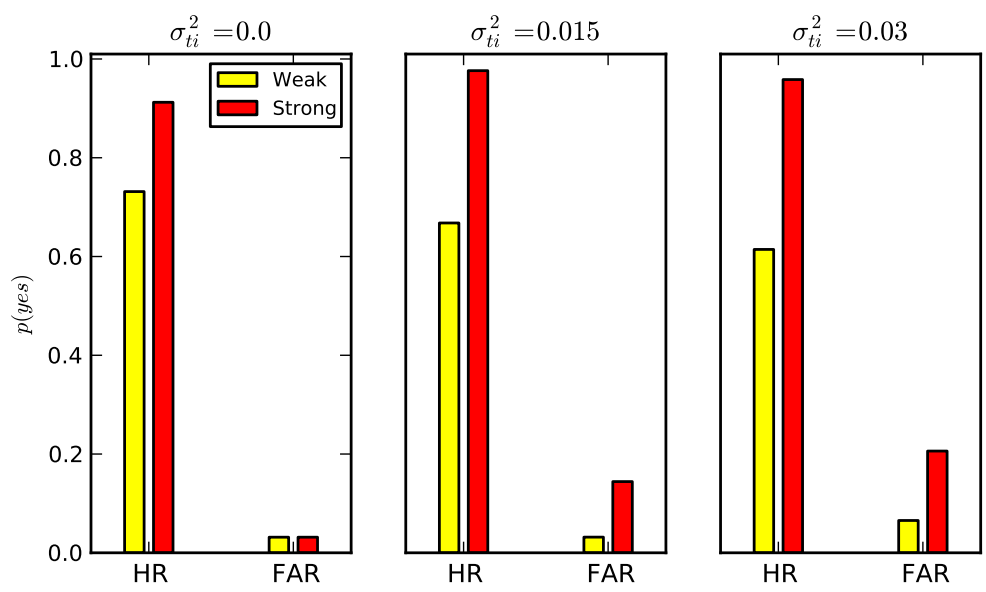

Figure 2.4: Graph displaying demonstrations of the effects of the item mismatch variability parameter $\left(\sigma_{t i}^{2}=0, .015, .03\right)$ on a mixed list of weak and strong pairs in associative recognition. Parameters were as follows: 15 weak pairs encoded with $r_{a s s o c}=1.0,15$ strong pairs encoded with $r_{a s s o c}=2.5, \mu_{t t}=1, \mu_{s s}=1, \sigma_{t t}^{2}=.025, \sigma_{s s}^{2}=.075$, and $\beta_{a s s o c}=.1$

\subsection{Background Noise}

From the description of the item mismatch variability parameter, it might seem as if large values of that parameter will always produce positive effects of list length and list strength. However, this is not the case. As noted by Murdock and Kahana (1993a, 1993b), a large contribution from pre-experimental memories can be sufficient to drown out differences between two conditions that vary in their level of item noise, such as differences in list length or list strength.

The effect of the background noise parameter on list length and list strength predictions can be seen in Figure 2.5. The item mismatch variability parameter was set at .03, which was seen to produce relatively large item noise effects in Figure 2.3. Background noise $(\beta)$ was varied for both item and associative recognition. The most obvious effect is that background noise degrades performance. However, as background noise increases, the increases in item noise with list length and list strength are relatively small compared to the interference already present in memory, and one can see that performance decreases at a smaller rate 
as list length or list strength are increased when the background noise present in memory is high.

While I have simplified the background noise contribution to a single parameter, the original parameterization describes it as follows:

$$
n\left(\mu_{s u}^{2} \sigma_{t i}^{2}+\mu_{t i}^{2} \sigma_{s u}^{2}+\sigma_{s u}^{2} \sigma_{t i}^{2}\right)
$$

where $n$ is the number of memories of the given stimulus class. Specifically, one can see that both $n$ and the item mismatch variability parameter $\sigma_{t i}^{2}$ determine the total background noise. Given that the item mismatch variability parameter varies across stimulus classes and that each stimulus class likely varies in its number of entries in memory, it is also reasonable for the background noise to vary across stimulus classes. In the fits, $\beta$ varies across stimulus classes but is constant across all other manipulations.

The demonstration that background noise can mask effects of list length and list strength is critical to our understanding of the sources of interference in recognition memory. Dennis and colleagues have previously argued that null effects of list length and list strength support the idea that there is no item noise in memory (Dennis \& Humphreys, 2001; Osth \& Dennis, 2013), whereas the presence of a significant contribution of background noise is a plausible alternative. Thus, null effects of list length and list strength do not necessitate a pure context noise model with no item noise. The model fits to a large number of experimental datasets allow me to distinguish between these possibilities. 


\section{Background Noise}

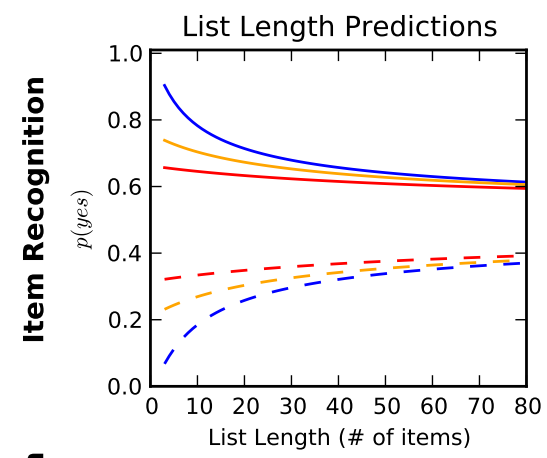

List Strength Predictions: HR and FAR List Strength Predictions: $d^{\prime}$
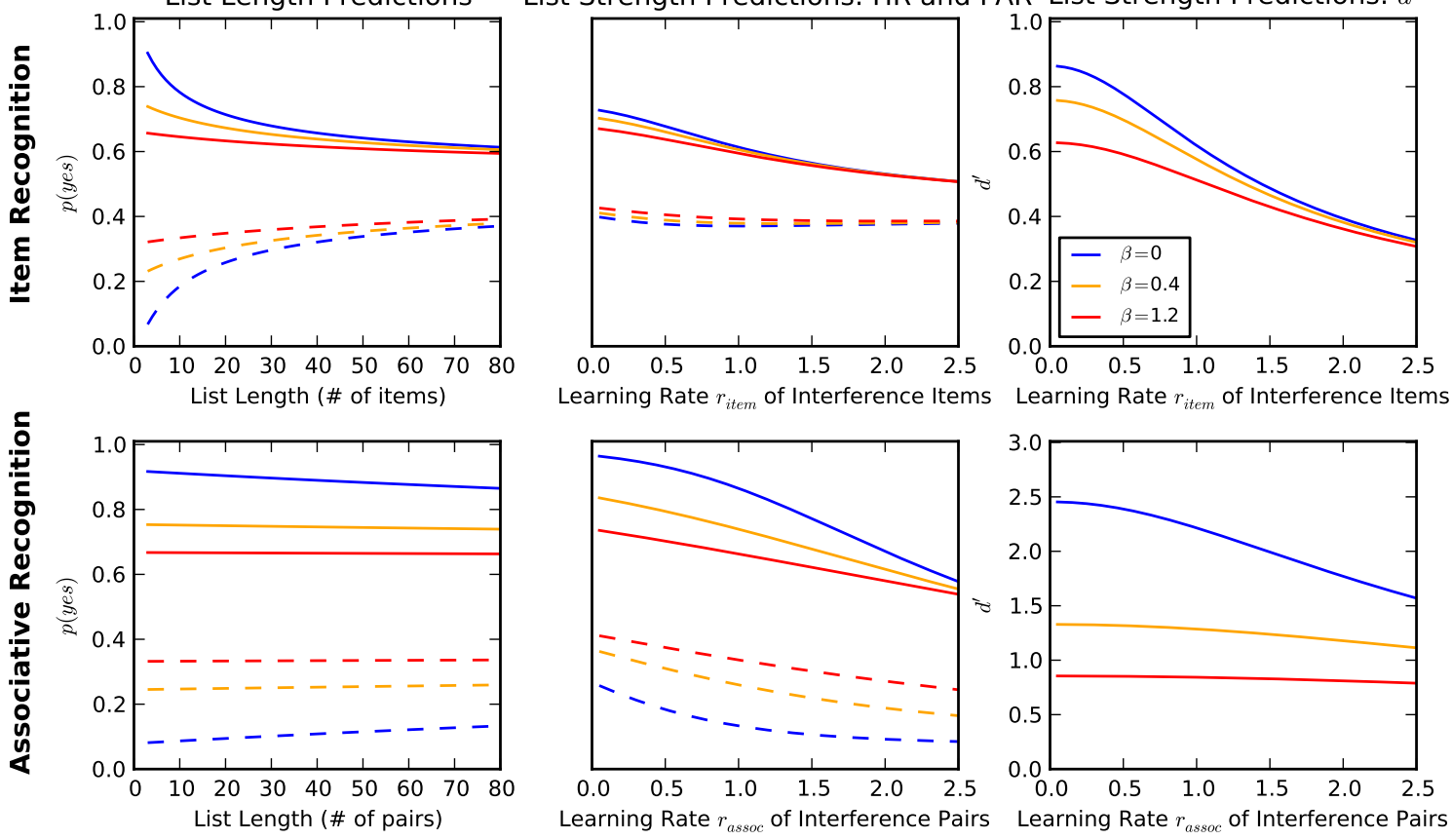

Figure 2.5: Graph displaying demonstrations of the effects of background noise both item recognition (top row) and associative recognition (bottom row). Background noise ( $\beta_{\text {item }}$ and $\beta_{\text {assoc }}$ ) was varied between $0, .4$, and 1.2. Depicted are simulations of a list length paradigm (left) and a list strength paradigm (middle column: HR and FAR predictions, right column: $d^{\prime}$ predictions). In the list length paradigm, the number of study list items was manipulated between 1 and 80 . In the list strength paradigm, 30 items were studied, half were baseline pairs studied with learning rate $r_{i t e m}=1.0$ and the other half were interference items studied with $r_{\text {item }}$ ranging between .05 and 2.5. The other parameters of the model were set as follows: $r_{i t e m}=1, \mu_{t t}=1, \mu_{s s}=1, \sigma_{t t}^{2}=.025, \sigma_{s s}^{2}=.075, \sigma_{t i}^{2}=.03$, $\rho=.001$. 


\section{Chapter 3 \\ The Model Fit}

As previously mentioned, multiple possibilities in the parameter values can be responsible for recognition memory being impervious to manipulations of list length and list strength on recognition memory performance for words. For instance, null effects of list length and list strength could be predicted by a model with context noise as the sole source of interference (like in the BCDMEM model) or they could be predicted with a high ratio of background noise to item noise (like in the TODAM models). Similarly, overall levels of performance can reflect a strong degree of learning or low contributions of interference. The fact that multiple parameter combinations can qualitatively predict similar outcomes implies that the resulting parameter estimates of the model will not be independent but correlated with each other. Thus, the modeling exercise requires a robust model fitting procedure.

To properly measure the parameters required to estimate the interference contributions to recognition memory, I fit the model within a hierarchical Bayesian framework. The virtues of hierchical Bayesian methods for fitting cognitive models (Lee, 2008, 2011; Lee \& Vanpaemel, 2008; Pooley, Lee, \& Shankle, 2011; Rouder \& Lu, 2005; Shiffrin, Lee, Kim, \& Wagenmakers, 2008; Vandekerckhove, Tuerlinckx, \& Lee, 2011) and in fitting data from recognition memory paradigms (Dennis et al., 2008; Pratte \& Rouder, 2011; Pratte, Rouder, \& Morey, 2010; Morey, Pratte, \& Rouder, 2008; Turner et al., 2013) have been well established. While traditional techniques such as minimizing the sum of squared deviations between the model's predictions and the data (approximate least squares) only provide point estimates for the model's parameters, one of the advantages of Bayesian analyses is 
that they quantify the uncertainty in the parameter estimates of the model as probability distributions over each parameter which are referred to as posterior distributions. Given that I am interested in quantifying the respective contributions of the interference contributions, properly measuring the uncertainty in these estimates is necessary.

Additionally, while it is common for modelers to aggregate across subjects and fit psychological models to only the group data, one problem is that fitting group estimates of the data often provides different parameter estimates than when the fits to the individual participants are averaged together (Estes \& Maddox, 2005). Bayesian analyses can overcome this problem by usage of hierarchical models that jointly estimate the parameters of the group and the individual participants. This is accomplished by establishing hyperparameters that represent the group level parameters in combination with individual participant

parameters. Each relevant model parameter (such as $r, \sigma_{t i}^{2}, \beta$, etc.) has its own set of hyperparameters, namely a mean and variance or precision parameters, that specify the prior distribution across all participants that each individual particiant's parameters are sampled from. The outcome of a hierarchical fit is posterior distributions on each model parameter for each participant along with posterior distributions for the hyperparameters of each model parameter that reflect the group-level parameters. Fitting individual participants in a modeling exercise such as this is critical, as there may be significant individual differences among participants in the magnitudes of the respective interference contributions.

\subsection{Datasets Included in the Model Fit}

Rather than fit each dataset separately, I imposed maximum constraint on the model by fitting all of the datasets simultaneously and constrained several hyperparameters across multiple datasets. Hyperparameters were allowed to vary across datasets where appropriate. For instance, the item mismatch variability parameter $\sigma_{t i}^{2}$ varies across stimulus classes, meaning that the Dennis et al. (2008) dataset, which employed words as stimuli, receives a different hyperdistribution for $\sigma_{t i}^{2}$ than for a dataset that used fractals as stimuli (such as Kinnell \& Dennis, 2012, Experiment 2). However, other datasets that used words, 
such as the Osth and Dennis (2013) and the DeCarlo (2007) studies, share the same $\sigma_{t i}^{2}$ hyperdistribution that represents word stimuli.

Here, I describe the datasets included in the model fit, their findings, the relevant hyperparameters they constrain, and briefly review the surrounding literature. A summary of all of the datasets used in the fitting can be seen in Table 3.1.

Table 3.1: Datasets included in the hierarchical Bayesian fit to the data.

\begin{tabular}{|c|c|c|c|c|c|}
\hline Dataset & Task & Stim. & $N$ & Resp. & Manip. \\
\hline $\mathrm{DC}-\mathrm{Ex} 1 \mathrm{~A}$ & $\mathrm{IR}$ & Words & 72 & 6 con. & $\begin{array}{l}\text { Word frequency: LF and HF } \\
\text { List length: } 20 \text { vs. } 80 \text { items }\end{array}$ \\
\hline DLK & IR & Words & 48 & $\mathrm{YN}$ & Word frequency: LF and HF \\
\hline & & & & & Unfilled vs. filled delay \\
\hline $\mathrm{KD}-\mathrm{Ex} 1$ & $\mathrm{AR}$ & Words & 40 & $\mathrm{YN}$ & List length: 24 vs. 96 pairs \\
\hline KD - Ex 2 & $\mathrm{IR}$ & Faces & 40 & $\mathrm{YN}$ & List length: 20 vs. 80 items \\
\hline $\mathrm{KD}-\operatorname{Ex} 3$ & IR & Fractals & 40 & $\mathrm{YN}$ & Same as above \\
\hline $\mathrm{KD}-\operatorname{Ex} 4$ & IR & Scenes & 40 & $\mathrm{YN}$ & $\begin{array}{l}\text { Same as above } \\
\text { List strength: }\end{array}$ \\
\hline ODK - Ex 1 & IR & Fractals & 88 & $\mathrm{YN}$ & 32 items $1 \mathrm{x}$ \\
\hline ODK - Ex 2 & IR & Faces & 96 & $\mathrm{YN}$ & $\begin{array}{l}\text { vs. } 16 \text { items } 1 \mathrm{x} 16 \text { items } 4 \mathrm{x} \\
\text { Same as above }\end{array}$ \\
\hline ODK - Ex 3 & $\mathrm{IR}$ & Scenes & 71 & $\mathrm{YN}$ & $\begin{array}{l}\text { Same as above } \\
\text { List strength: }\end{array}$ \\
\hline OD - Ex 1 & $\mathrm{AR}$ & Words & 80 & $\mathrm{YN}$ & 32 pairs $1 \mathrm{x}$ \\
\hline
\end{tabular}

Notes: Stim. = stimulus, $N=$ number of participants, resp. $=$ response type collected in the experiment, manip. = manipulations used in the experiment. DC $=$ DeCarlo (2007), DLK = Dennis, Lee, \& Kinnell (2008), KD = Kinnell \& Dennis (2012), ODK = Osth, Dennis, \& Kinnell (in press), OD = Osth \& Dennis (2013), IR = item recognition, $\mathrm{AR}=$ associative recognition, $\mathrm{YN}=$ yes-no recognition, 6 con $=6$ point cỏlfidence rating scale. 


\subsubsection{The ROC in item recognition}

As described earlier, the slope of the z-transformed ROC is almost uniformly less than one in the recognition memory literature (Egan, 1958; Glanzer et al., 1999; Heathcote, 2003; Ratcliff et al., 1992, 1994), a finding which has been used to advocate for the unequal variance signal detection model (Wixted, 2007; Starns \& Ratcliff, 2014). The ubiquity of the unequal variance interpretation of zROC slopes is further supported by evidence from response time models. Starns, Ratcliff, and McKoon (2012) found that the Ratcliff diffusion model (Ratcliff, 1978; Ratcliff, Van Zandt, \& McKoon, 1999) could only fit the zROC slopes from a binary ROC paradigm (in which participants give yes or no responses, but the relative proportions of targets and lures are manipulated across conditions) if the variability of the drift rates for targets was larger than the variability for lures. More recently, Starns and Ratcliff (2014) fit a large number of recognition memory datasets that lacked complete ROC functions and found that a diffusion model with higher drift rate variability for targets received better support than a diffusion model with equal variance across the drift rates for targets and lures.

As mentioned previously, unequal variance between the target and lure distributions can be produced in the model by choosing the appropriate values of the item and context match variability parameters $\sigma_{t t}^{2}$ and $\sigma_{s s}^{2}$. However, many of the datasets listed below lack the necessary data to constrain these parameters, in that they did not include manipulations of target vs. lure proportions or confidence ratings. It was for this reason that I additionally selected a relatively simple ROC experiment to constrain these parameters, namely Experiment 1A of DeCarlo (2007). This experiment tested native English participants for item recognition of both high and low frequency words using six point confidence ratings. To model this dataset, five hyperparameters were selected for the response criteria required to make the confidence ratings that were not employed in any of the other datasets. Differences between the word frequency classes were modeled by usage of the context mismatch variability parameter $\rho$ : separate hyperparameters were used for both low and high frequency words. The presentation time for the stimuli was lower than for the other datasets (one sec- 
ond per stimulus), so this dataset was alotted its own hyperparameters for the learning rate $r$. Given that this experiment used immediate testing, the mean context match parameter $\mu_{s s}$ was fixed at 1.

\subsubsection{The List Length Paradigm}

Manipulations of the length of a study list have been a large constraint on models of memory, including models of recognition memory (Chappell \& Humphreys, 1994; Clark \& Gronlund, 1996; Dennis \& Humphreys, 2001; Gillund \& Shiffrin, 1984; Johns, Jones, \& Mewhort, 2012; McClelland \& Chappell, 1998; Shiffrin et al., 1990; Shiffrin \& Steyvers, 1997), free recall (G. D. A. Brown, Neath, \& Chater, 2007; Davelaar, Goshen-Gottstein, Ashkenazi, Haarmann, \& Usher, 2005; Farrell, 2012; Polyn, Norman, \& Kahana, 2009; Raaijmakers \& Shiffrin, 1981; Sederberg, Howard, \& Kahana, 2008), and serial recall (Botvinick \& Plaut, 2006; G. D. A. Brown et al., 2000; Henson, 1998; Farrell, 2012; Lewandowsky \& Murdock, 1989). In recognition memory, the earliest demonstration of the detrimental effect of list length on recognition memory performance was found by Strong (1912). In the several decades that followed, the list length effect was replicated extensively in both item (Bowles \& Glanzer, 1983; Cary \& Reder, 2003; Gillund \& Shiffrin, 1984; Gronlund \& Elam, 1994; Murnane \& Shiffrin, 1991a; Nobel \& Shiffrin, 2001; Ratcliff \& Murdock, 1976; Underwood, 1978) and associative recognition (Clark \& Hori, 1995; Nobel \& Shiffrin, 2001) and has been used as support for pure item noise models of recognition memory (Clark \& Gronlund, 1996; Gillund \& Shiffrin, 1984).

However, as noted by Dennis and Humphreys (2001), a number of confounds exist in the previously published list length designs that may be artifactually causing a list length effect in recognition memory performance that I will briefly summarize here. First, if participants are tested immediately after completion of the study list, the average retention interval for items in the long list is longer than that of the short list. This confound can be overcome by equating the retention intervals across the two list length conditions by using a period of filler activity after the short study list is complete. Another confound is the fact that when immediate testing is used after a long list, participants may be more inclined to use 
a context representation that strongly favors the end-of-list items, rather than reinstating a list-wide context, a confound which can be overcome by having participants partake in additional filler task activity. An additional confound is that attention is likely to decrease through the duration of the study list, producing weaker encoding for the late list items relative to the early list items, a point which was first raised by Underwood (1978). This confound can be remedied by comparing items from equivalent serial positions from both the short and long list conditions.

When all of these confounds have been controlled, the investigations by Dennis and colleagues have found no effect of list length on performance when words are used as stimuli both in item recognition (Dennis \& Humphreys, 2001; Dennis et al., 2008; Kinnell \& Dennis, 2011) and associative recognition (Kinnell \& Dennis, 2012, Experiment 1). Other investigations which have found no effect of list length on performance in item recognition. Schulman (1974) found no difference in 2AFC recognition performance between study lists of lengths 25, 50, and 100 items when the retention intervals and test positions were equated across the conditions. Jang and Huber (2008) found no difference between list lengths of 6 and 24 items in a $2 \mathrm{AFC}$ recognition task that interpolated between two lists that were later tested for free recall. Murnane and Shiffrin (1991a, Experiment 3) found no effect of list length on yes-no recognition performance when study-test lag was controlled.

While some might conclude that the controls employed in these list length paradigms might be sufficient to eliminate any effect of list length in recognition memory performance, this is not the case. Kinnell and Dennis (2012) tested novel non-linguistic stimuli, specifically images of fractals, faces, and natural scenes, in single item recognition using the same controls employed in the other investigations. Significant effects of list length were found for fractals and faces but no effect of list length was found for natural scenes. Kinnell and Dennis (2012) posited that the differences associated with the different stimulus classes may be attributed to different levels of item noise, with word stimuli and natural scenes being exempt from item noise at retrieval while faces and fractals suffer from item noise, possibly due to having more distributed item representations (I return to the issue of how different stimulus classes can suffer from different degrees of item noise in the General Discussion). 
Included in the model fit are five experiments using the list length paradigm: the dataset of Dennis et al. (2008) along with the four experiments by Kinnell and Dennis (2012). All of the experiments use two list length conditions which all employ a 1:4 list length ratio from the short list to the long list. Additionally, all of the experiments only test the first 20 items (first 32 pairs for the associative recognition experiment) on the study list to make the test lists across the two list length conditions comparable. The five datasets comprise all of the stimuli that are employed in the model fit: words are employed in the study of Dennis et al. (2008) and the associative recognition experiment by Kinnell and Dennis (2012, Experiment 1). Faces, fractals, and natural scenes were compared using separate experiments by Kinnell and Dennis (2012, Experiments 2-4, respectively).

The dataset of Dennis et al. (2008) was somewhat more extensive than the other datasets, as it also manipulated the length of a post study list delay period in addition to two different levels of word frequency. In the unfilled delay condition, recognition testing began immediately after the long list and 3 minutes after the end of the short list. In the filler condition, in contrast, recognition testing began an additional 8 minutes after the end of the long list and 11 minutes after the end of the short list. To capture the different levels of performance for each delay, separate hyperparameters for the mean context strength parameter $\mu_{s s}$ were allocated: one for the long list in the unfilled condition to allow for the possibility of poor contextual reinstatement, and another for both list length conditions in the filler task condition. To capture the effects of word frequency, the same context mismatch variability hyperparameters used in the fit to the dataset of DeCarlo (2007) were used to capture the effects of low and high frequency words in this dataset.

To test for the hypothesis that the different stimulus classes are subject to different degrees of item noise, separate hyperparameters for the item mismatch variability parameter $\sigma_{t i}^{2}$ were allowed for each stimulus class. Osth et al. (2014) argued that the detrimental effects of list length and list strength observed with specific non-linguistic stimuli are too small to be accommodated by a pure item noise model and that these effects may be being mitigated by background noise from the memory system. For this reason, different hyperparameters for the background noise parameter $\beta_{\text {item }}$ were allowed for each stimulus 
class. Additionally, separate background noise hyperparameters were allowed for word pairs in associative recognition to allow for the possibility that many more inter-item bindings are being stored in the co-occurrence tensor than item-context bindings in the occurrence matrix. Since the experiments of Kinnell and Dennis (2012) also used filler tasks that are of the same length as the filler task of Dennis et al. (2008), the same hyperparameters for the mean context strength were shared across all of these experiments.

\subsubsection{The List Strength Paradigm}

The null list strength effect was initially discovered by Ratcliff et al. (1990), who found that recognition performance for weak items was not harmed when they were accompanied by strong items on a study list and that strong items did not benefit from being accompanied by weak items relative to strong items. The null list strength effect was found in all seven of their experiments, regardless of whether the strengthening occurred via massed study, massed repetitions, or spaced repetitions. The list strength paradigm was extensively revisited in the two decades to follow, resulting in several replications of the null list strength effect in item recognition (Hirshman, 1995; Kahana, Rizzuto, \& Schneider, 2005; Murnane \& Shiffrin, 1991a, 1991b; Ratcliff et al., 1994, 1992; Shiffrin, Huber, \& Marinelli, 1995; Yonelinas, Hockley, \& Murdock, 1992) and was recently demonstrated in associative recognition by Osth and Dennis (2013) using both yes/no and 2AFC testing. The finding of the null list strength effect has become a canonical constraint on recognition memory models (Chappell \& Humphreys, 1994; Dennis \& Humphreys, 2001; Johns et al., 2012; McClelland \& Chappell, 1998; Norman \& O’Reilly, 2003; Shiffrin et al., 1990; Shiffrin \& Steyvers, 1997).

The vast majority of the investigations that found no effect of list strength on recognition memory performance used single words or word pairs as study and test stimuli. Following the investigation of Kinnell and Dennis (2012), Osth et al. (2014) tested images of fractals, faces, and natural scenes for the presence of a list strength effect while simultaneously employing the list length controls advocated by Dennis and colleagues ${ }^{5}$. A significant list

\footnotetext{
${ }^{5}$ As noted by Osth et al. (2014), while the controls for differences in retention interval, attention, and contextual reinstatement are not commonly applied in list strength paradigms, they can similarly contribute to the artifactual finding of a list strength effect.
} 
strength effect was found for fractals with both yes/no and 2AFC testing while null effects of list strength were found for faces and scenes (although a significant list strength effect was found using artificial faces by Norman et al., 2008). Osth et al. (2014) used this finding to support the hypothesis of Kinnell and Dennis (2012) that novel non-linguistic stimuli may be more susceptible to the effects of item noise.

An additional regularity of the list strength paradigm is the strength based mirror effect, in which strengthening a set of list items results in higher hit rates for those items along with a lower false alarm rate. Hirshman (1995) found this pattern to be ubiquitous in the early published list strength paradigms, and several investigations have replicated the effect in both item recognition (Criss, 2006, 2009, 2010; Hockley \& Niewiadomski, 2007; Singer, 2009; Starns, Ratcliff, \& White, 2012; Starns et al., 2010; Starns, White, \& Ratcliff, 2012; Stretch \& Wixted, 1998b) and associative recognition (Clark \& Shiffrin, 1992; Hockley \& Niewiadomski, 2007; Osth \& Dennis, 2013). Furthermore, all of the non-linguistic stimuli in the study by Osth et al. (2014) exhibited a strength based mirror effect.

Included in the model fit are four experiments using the list strength paradigm: the datasets of Osth et al. (2014) and Osth and Dennis (2013). All of these experiments compared lists of 32 unique items or pairs of items across two conditions: a pure weak condition where all items or pairs were presented once, along with a mixed list condition where half of the items or pairs were presented once and the other half were presented four times. To make the tests of both list conditions comparable, all repetitions in these studies occurred after all of the unique items were presented once. Fractals, faces, and natural scenes were the stimuli of Osth et al. (2014, Experiments 1-3, respectively) and word pairs were employed in the study of Osth and Dennis (2013). Only the yes-no data from these investigations were employed. $2 \mathrm{AFC}$ predictions were not able to be obtained analytically as there is no analytic expression for the difference of two non-central chi-square distributions.

In all experiments, hit rates for strong items or pairs greatly exceeded those of weak items or pairs. This was accommodated by allocating a separate learning rate $r_{\text {item }}$ or $r_{\text {assoc }}$ for the items or pairs that were presented four times. To guarantee that learning rates for strong items were exceeded those for weak items, the samples of the strong learning rates 
were added to the weak learning rates. The value of the strong learning rates along with the value of the item mismatch variability parameter influence the magnitude of the list strength effect.

One aspect of the associative recognition dataset that distinguishes it from the item recognition datasets is that the test lists in the mixed lists comprised rearranged pairs constructed from both weak (once presented) and strong (four times presented) pairs. Several studies have made such a comparison and found equivalent false alarm rates to both weak and strong pairs in young adults (Buchler et al., 2008; Cleary, Curran, \& Greene, 2001; Gallo, Sullivan, Daffner, Schacter, \& Budson, 2004; Kelley \& Wixted, 2001; Mickes, Johnson, \& Wixted, 2010; Osth \& Dennis, 2013) ${ }^{6}$, which can be considered a broken within-list strength based mirror effect, although some studies have found reduced false alarm rates for strong pairs under specific conditions such as delayed responding or substantial strength differences between weak and strong pairs (Light et al., 2004; Malmberg \& Xu, 2007; Xu \& Malmberg, 2007). A unique aspect of the Osth and Dennis dataset is that both an intact and broken strength based mirror effect were observed: false alarm rates were lower in mixed lists than in pure weak lists (an intact across-list strength based mirror effect), but false alarm rates to weak and strong pairs were equivalent in the mixed list (a broken within-list strength based mirror effect). As was previously mentioned, the false alarm rates between weak and strong pairs is an additional constraint on the item mismatch variability parameter $\sigma_{t i}^{2}$.

\subsubsection{Response Criteria}

In the Turner et al. (2013) model fit, the criteria of the model were fixed at 0.0, which reflects the point on the log likelihood ratio scale where an item is equally likely to be a target or a lure. Nonetheless, some recent evidence has suggested that there is substantial variability in criteria across participants. Aminoff et al. (2012) found reliable individual differences in

\footnotetext{
${ }^{6}$ Higher false alarm rates to strong pairs have been observed in older adults (Buchler, Faunce, Light, Gottfredson, \& Reder, 2011; Light, Patterson, Chung, \& Healy, 2004). While one possibility for this result is that older adults suffer from more item noise at retrieval, another possibility is that they rely more on item information in the associative recognition task than in younger adults, possibly due to a poorer ability to encode and use associations (Naveh-Benjamin, 2000).
} 
criteria across participants and found they were predicted by such psychometric variables as personality traits and affect. Kantner and Lindsay (2012) found that individual differences in response criteria were stable across recognition tests that were up to a week apart, although they varied somewhat across different stimulus materials.

Thus, I allowed for individual differences in the decision criterion parameter by having each participant's criterion sampled from a set of hyperparameters. Additionally, given the differences among different experiments in terms of the perceived difficulty of the stimulus materials, I have allowed different hyperparameters for each stimulus class to be used. Criteria were not allowed to vary across conditions or across trials.

\subsection{The Hierarchical Bayesian Model}

It is quite common to express hierarchical Bayesian analyses as graphical models (Jordan, 2004). However, due to the large number of datasets being used in the fit, the many parameters of the recognition memory model being fit to the data in addition to the fact that several parameters are constrained across the fits, a graphical model would be too large and cumbersome to be useful to the reader. Instead, I have presented all parameters of the hierarchical fit that receive their own set of hyperparameters (mean and variance/precision) in Table 3.2.

The hierarchical model was fit using JAGS software (Plummer, 2003). The data from

all of the experiments were each individual participant's raw response counts to targets and lures for each condition. All experiments that used yes-no response formats were fit via a binomial likelihood function, whereas the experiment that used six point confidence ratings (DeCarlo, 2007) was fit using a multinomial likelihood function. The rate parameters that entered the binomial likelihood functions were constructed by calculating the area above the response criterion of the log likelihood ratio distributions for targets and lures for each condition. For the confidence ratings, the multinomial rate parameters were generated by calculating the area between the criteria for the middle responses, the area above the highest criterion for the highest confidence rating, and the area below the lowest criterion for the 
lowest criterion.

One of the advantages of the Bayesian approach is the ability to restrict the parameter space via a specified prior distribution to reflect a priori beliefs about how the parameter is distributed (Vanpaemel \& Lee, 2012). However, given that this model has not been previously fit to data, I use non-informative priors instead which place approximately equal likelihood over all possible values in the parameter space. For participant parameters that are bounded between 0 and $1\left(r\right.$ and $\left.\mu_{s s}\right)$, parameters were sampled from beta distributions that were reparameterized in terms of the mean $(\lambda)$ and variance $(\nu)$ parameters of the beta distribution. This reparameterization is achieved from the initial parameters $\alpha$ and $\beta$ by setting $\alpha=\lambda \nu$ and $\beta=(1-\nu) \lambda$. Non-informative priors on these parameters were as follows:

$$
\begin{aligned}
& \lambda \sim \operatorname{Beta}(.5,2) \\
& \nu \sim \operatorname{InverseGamma}(.1, .1)
\end{aligned}
$$

For parameters that are bounded between 0 and $\infty\left(\sigma_{t t}^{2}, \sigma_{s s}^{2}, \sigma_{t i}^{2}, \rho\right.$, and $\left.\beta\right)$, participant parameters were sampled from lognormal distributions. For parameters that are bounded between $-\infty$ and $\infty$ (the criterion parameters), participant parameters were sampled from normal distributions. Non-informative priors on the mean $(\omega)$ and precision $(\xi)$ of the normal and lognormal distributions were specified as follows:

$$
\begin{aligned}
\omega & \sim \operatorname{Normal}(0, .001) \\
\xi & \sim \operatorname{InverseGamma}(.1, .1)
\end{aligned}
$$

where the parameters of the normal distribution are the mean and precision. The results of the model fit are based on twenty chains, each consisting of 10,000 samples after 3,500 burn-in samples were discarded. 
Table 3.2: All hyperparameters included in the hierarchical model.

\begin{tabular}{|c|c|c|c|}
\hline Param. & Number & Cond. & Datasets \\
\hline \multirow{8}{*}{$r_{i t e m}$} & 1 & Words (1 sec.) & $\mathrm{DC}$ \\
\hline & 2 & Words (3 sec.) & DLK \\
\hline & 3 & Fractals $1 \mathrm{x}$ & KD Ex 2; ODK Ex 1 \\
\hline & 4 & Fractals $4 \mathrm{x}$ & ODK Ex 1 (Mixed list cond.) \\
\hline & 5 & Faces $1 \mathrm{x}$ & KD Ex 3; ODK Ex 2 \\
\hline & 6 & Faces $4 \mathrm{x}$ & ODK Ex 2 (Mixed list cond.) \\
\hline & 7 & Scenes $1 \mathrm{x}$ & KD $\operatorname{Exp} 4 ;$ ODK Ex 3 \\
\hline & 8 & Scenes $4 \mathrm{x}$ & ODK Ex 3 (Mixed list cond.) \\
\hline \multirow{3}{*}{$r_{\text {assoc }}$} & 1 & Pairs $1 \mathrm{x}$ & KD Ex $1 ;$ OD \\
\hline & 2 & Pairs 4x & OD (Mixed list cond.) \\
\hline & 1 & Short delays (3.5 min) & ODK Ex $1,2,3 ;$ OD \\
\hline \multirow[t]{2}{*}{$\mu_{s s}$} & 2 & Long delays (8 min) & DLK; KD Ex $1,2,3,4$ \\
\hline & 3 & Long list, no filler & DLK \\
\hline$\sigma_{t t}^{2}$ & 1 & All & All datasets \\
\hline$\sigma_{s s}^{2}$ & 2 & All & All datasets \\
\hline \multirow{4}{*}{$\sigma_{t i}^{2}$} & 1 & Words & DLK; DC; KD Ex 1; OD \\
\hline & 2 & Fractals & KD Ex 2; ODK Ex 1 \\
\hline & 3 & Faces & KD Ex 3; ODK Ex 2 \\
\hline & 4 & Scenes & KD Ex 4; ODK Ex 3 \\
\hline \multirow{3}{*}{$\rho$} & 1 & LF words & DLK; DC \\
\hline & 2 & HF words & DLK; DC \\
\hline & 1 & Words & DLK; DC \\
\hline \multirow{3}{*}{$\beta_{i t e m}$} & 2 & Fractals & KD Ex 2; ODK Ex 1 \\
\hline & 3 & Faces & KD Ex 3; ODK Ex 2 \\
\hline & & & Continued on next page \\
\hline
\end{tabular}


Table 3.2 - continued from previous page

\begin{tabular}{llll}
\hline Param. & Number & Cond. & Datasets \\
\hline \multirow{3}{*}{$\beta_{\text {assoc }}$} & 4 & Scenes & KD Ex 4; ODK Ex 3 \\
& 1 & Pairs & KD Ex 1, OD \\
& $1-5$ & Confidence & DC \\
6 & & DLK \\
$\Phi$ & 7 & Fractals & KD Ex 2; ODK Ex 1 \\
& 8 & Faces & KD Ex $3 ;$ ODK Ex 2 \\
9 & Scenes & KD Ex 4; ODK Ex 3 \\
10 & Pairs & KD Ex 1; OD
\end{tabular}

Notes: Param. = parameter, cond. = condition, DC = DeCarlo (2007), DLK = Dennis, Lee, \& Kinnell (2008), KD = Kinnell \& Dennis (2012), ODK $=$ Osth, Dennis, \& Kinnell (in press), OD = Osth \& Dennis (2013), $1 \mathrm{x}=$ once presented, $4 \mathrm{x}=$ four times presented. Note that each parameter receives its own mean and variance/precision parameter - see the text for details.

\subsection{Analysis of the Model Fit}

In the presentatation of the fit of the model to the data, I present both group level predictions and predictions for the individual participants. Group level predictions were derived from the means of the hyperparameters which correspond to group-level estimates of the relevant parameters. For parameters that were lognormally distributed, the hypermean $\omega$ parameters were transformed as $e^{\omega}$, which is both the geometric mean and median of the lognormal distribution ${ }^{7}$. For predictions at the level of individual participants, samples were taken from the entire hyperdistribution (both the mean and variance/precision parameters)

\footnotetext{
${ }^{7}$ The arithmetic mean of the lognormal distribution is $e^{\omega+1 / 2 \xi^{2}}$. I preferred usage of the geometric mean/median $e^{\omega}$ due to the strong degree of skew in the lognormal distribution, which makes the arithmetic mean a worse measure of central tendency.
} 
on each sample and those parameters were used to generate model predictions. For analysis of the individual participants' interference contributions, individual participant parameters were used.

Where necessary, inferential statistics were performed on model parameters by taking the difference between the means of the hyperparameters and evaluating the proportion of samples that are above zero, which measures the probability of a difference between the two model parameters. Additionally, all density estimates on the posterior distributions were performed using Gaussian kernel density estimation.

\subsection{Parameter Estimates}

Posterior distributions of the group means for each parameter can be seen in Figure 3.1. For each parameter, clear differences can be seen across each stimulus class and experimental manipulation. The learning rate $r$ is highest for strong items in the list strength experiments. Learning rates are also lower for fractals and faces than for word pairs and scenes, and appear to be lowest for the words in the DeCarlo (2007) dataset, which used a brief presentation rate. Context match $\mu_{s s}$ parameters vary by the duration of filler activity, with the lowest values seen for the 8 minute filler activity and higher values for the 3.5 minute filler activity and the long list, no filler condition of Dennis et al. (2008) to reflect poor contextual reinstatement. The context mismatch parameter $\rho$ varies by word frequency, with higher values seen for high frequency words. A slightly negative bias in the criteria $(\Phi)$ can be seen for all stimulus classes except for scenes, which exhibit a more conservative bias.

Both the item mismatch parameter $\sigma_{t i}^{2}$ and the background noise parameter $\beta$ vary by stimulus class. Difference distributions for these parameters can be seen in Figure 3.2. The highest values for the item mismatch variability parameter are for fractals and faces. For fractals, $98.0 \%$ of the fractals minus words difference distribution lies above zero. For the faces minus words comparison, $99.9 \%$ of the difference distribution lies above zero. Images of natural scenes do not appear to differ significantly from words, as only $36.3 \%$ of the scenes minus words difference distribution lies above zero. For the background noise parameter, 


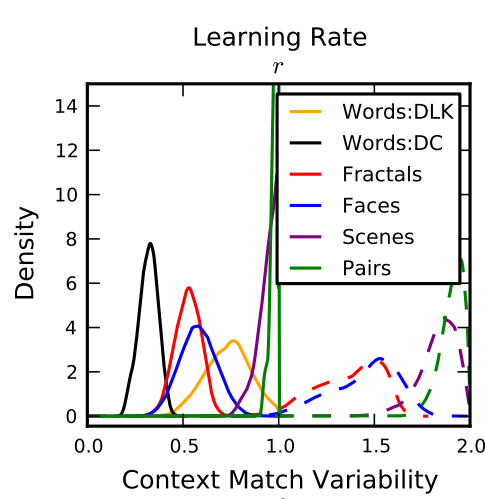

\section{Parameter Estimates}
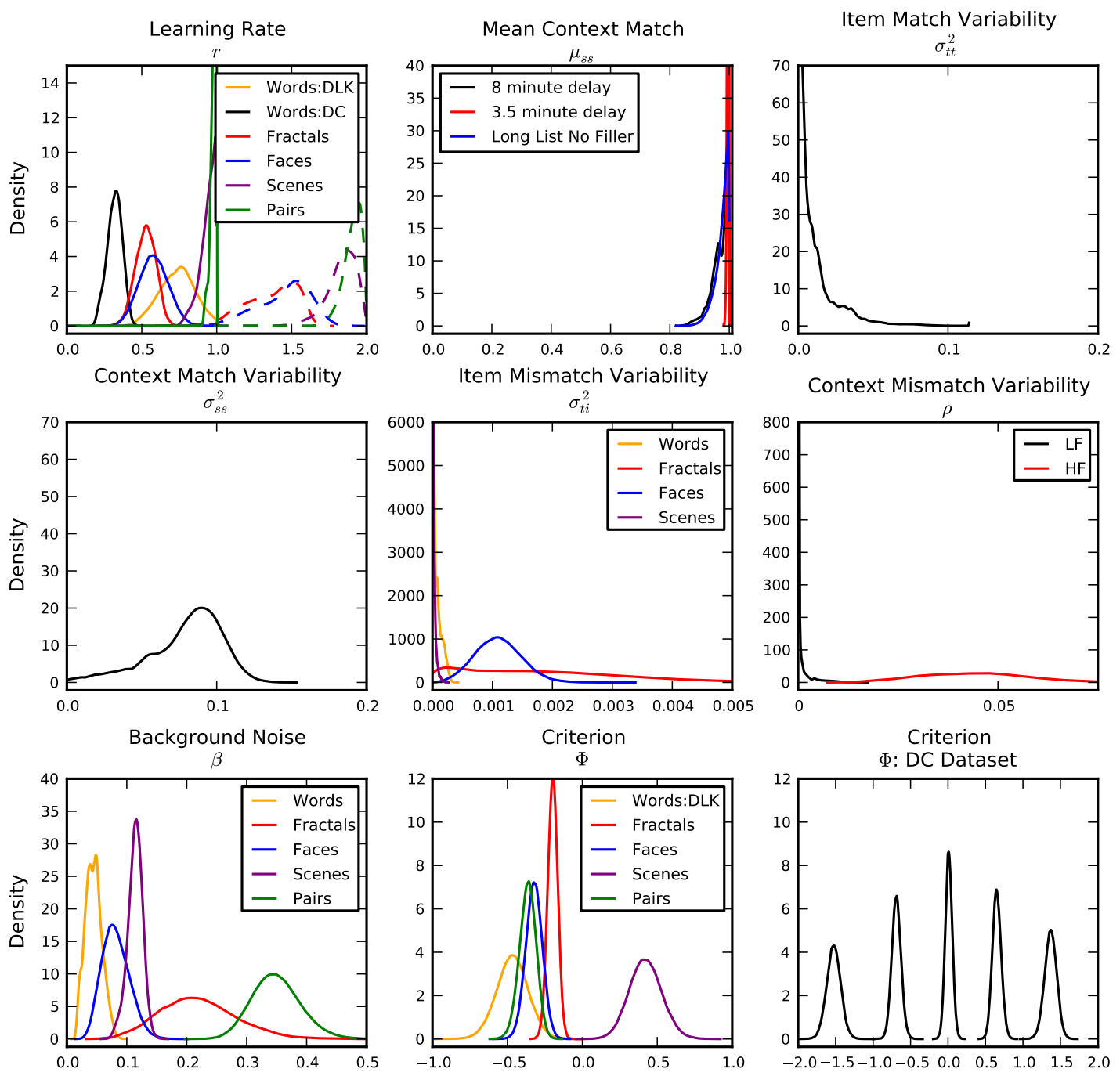

Figure 3.1: Posterior distributions for all hyperparameters in the omnibus fit. Depicted parameters are the learning rate $r$ (learning rates for strong items/pairs are the sum of the weak and strong learning samples and are indicated by dashed lines), the mean context match $\mu_{s s}$, the variance in the item match $\sigma_{t t}^{2}$, the variance in the context match $\sigma_{s s}^{2}$, the item mismatch variance $\sigma_{t i}^{2}$, the context mismatch variance $\rho$, the background noise $\beta$, along with the decision criteria $\Phi$. Note: DLK = Dennis, Lee, and Kinnell (2008) dataset, DC = DeCarlo (2007) dataset. 


\section{Parameter Differences}
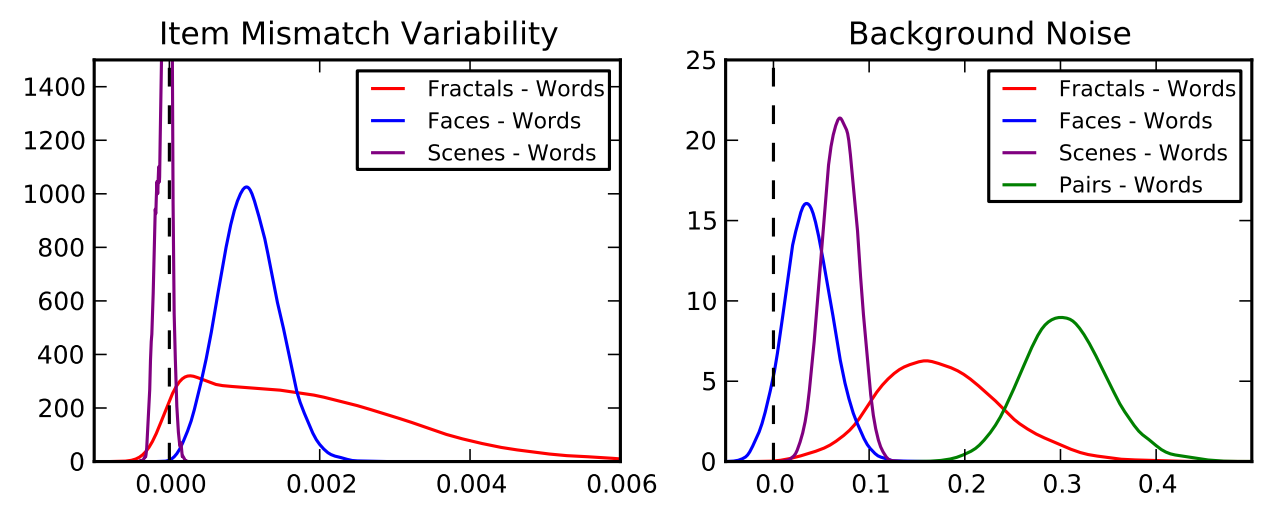

Figure 3.2: Density estimates of the differences between the means of the hyperparameters between words and other stimulus classes for both the item mismatch variability parameter $\sigma_{t i}^{2}$ (left) and the background noise parameter $\beta$ (right).

fractals, scenes, and pairs differ significantly from words, with $100 \%$ of the area of the difference distribution lying above zero for each comparison. While faces appear to exhibit more background noise than words, $93.5 \%$ of the area of the faces minus words difference distribution lies above zero.

\subsection{Model Fit}

While the primary purpose of fitting the model to data was to measure the magnitudes of the different interference contributions, interpretation of the model parameters is also reliant on the model achieving a good quantitative fit to the data. In this section, I present the model's predictions alongside both the group and individual participant data. For the group data, rather than compare the hit and false alarm rates generated from frequentist methods, I separately estimated the hit and false alarm rates for each dataset using simple hierarchical models and depict the predicted rates from the group mean parameters. Details of how the binomial and multinomial rates were estimated can be seen in Appendix C. For the individual participant data, the raw hit and false alarm counts from each participant are depicted and the model's predictions were generated from both the means and variance 
Fit to DeCarlo (2007)
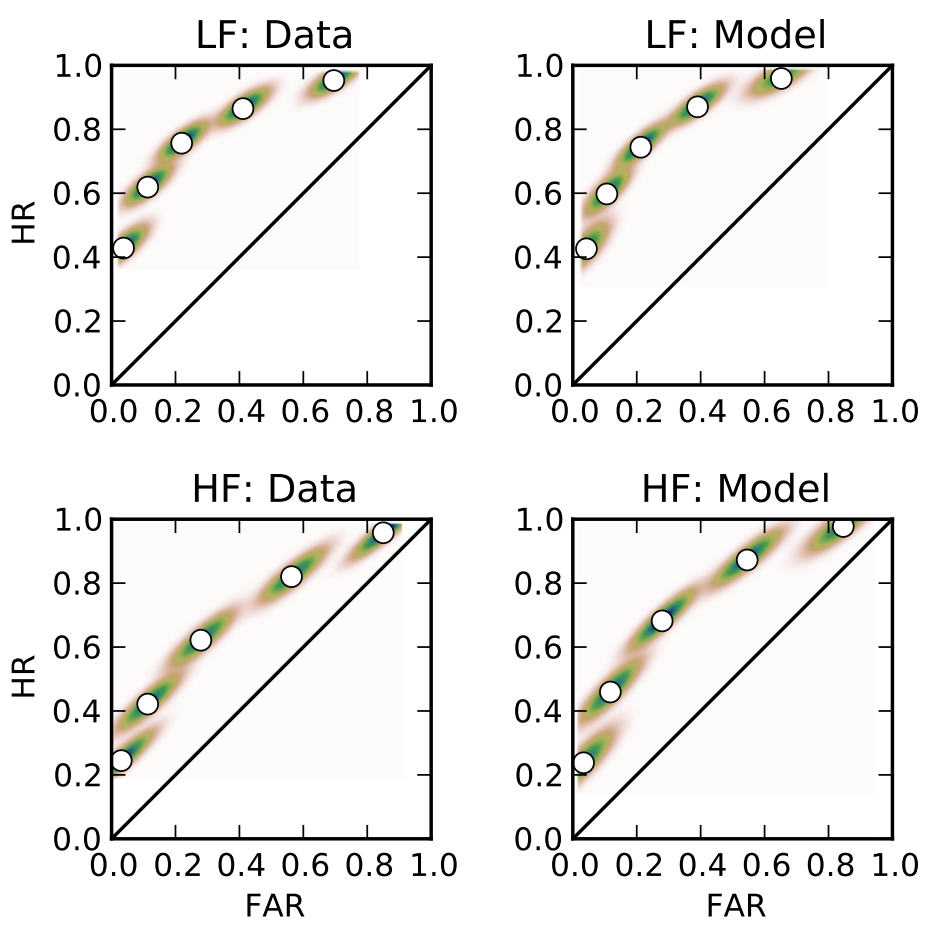

Figure 3.3: Density estimates of the ROC function for both the data of DeCarlo (2007, left) and model (right) for low (top) and high (bottom) frequency words. The circles indicate the median of the hit and false alarm rate posterior distributions for each ROC point.

parameters of each hyperparameter. Individual participant data and the model predictions are depicted using scatterplots in ROC space.

\subsubsection{Word Frequency and Confidence Ratings: Fit to DeCarlo (2007)}

The fit to the DeCarlo (2007) dataset can be seen in Figure 3.3 for the standard ROC and Figure 3.4 for the z-transformed ROC. To generate a density estimate of the ROC function, the density was estimated on all of the points of the confidence based ROC simultaneously. Inspection of the graphs reveals that the model exhibits a close correspondence to the experimental data, with better predicted performance for LF words than HF words.

The model misses slightly on the zROC slopes: predicted zROC slopes for the median zROC points for the data are .81 and .83 for LF and HF words whereas the model pro- 
duced zROC slopes of .91 and .95 for LF and HF words. This may be because inspection of Figure 3.4 reveals that the data's zROCs for HF words are slightly curvilinear, whereas the model is only capable of producing linear zROC slopes. Curvilinear zROC slopes have been attributed to various factors, such as the presence of recollection (Yonelinas, 1994), probability mixtures of encoded and non-encoded items (DeCarlo, 2002), and decision noise (Ratcliff \& Starns, 2009). I would like to emphasize that the primary purpose of including this dataset in the omnibus fit was not to discriminate between different theoretical explanations of ROC functions, but to constrain the parameters of the model that are principally responsible for producing unequal variance between the target and lure distributions, namely the variance in the item and context match parameters $\sigma_{t t}^{2}$ and $\sigma_{s s}^{2}$. Inspection of Figure 3.1 reveals that the parameters are well constrained by the data, as their posterior distributions appear within only a limited range of their prior distributions, which are broad and non-informative.

Fits to the individual participant data can be seen in Figure 3.5. One can see that the density of the model's predictions closely follows the density of the individual participant responses.

\subsubsection{List Length, Word Frequency, and Study-Test Delay: Fit to Dennis, Lee, and Kinnell (2008)}

The fit to the group data of Dennis et al. (2008) can be seen in Figure 3.6. For this dataset and all remaining datasets, the density estimates of the posterior distributions of both the rates estimated from the data along with the model's predicted rates are depicted using teardrop plots.

The model achieves an excellent fit to the data, with the posteriors distributions of the model's predictions aligning very closely with the posterior of the group data. As mentioned previously, this dataset exhibited a small list length effect in the no filler condition while there was no effect of list length in the filler condition, similar to what is seen in the data. Dennis et al. (2008) argued that when study lists are immediately followed by a test list, participants might be more likely to use an end-of-list context than reinstate a list-wide 


\section{Fit to DeCarlo (2007)}
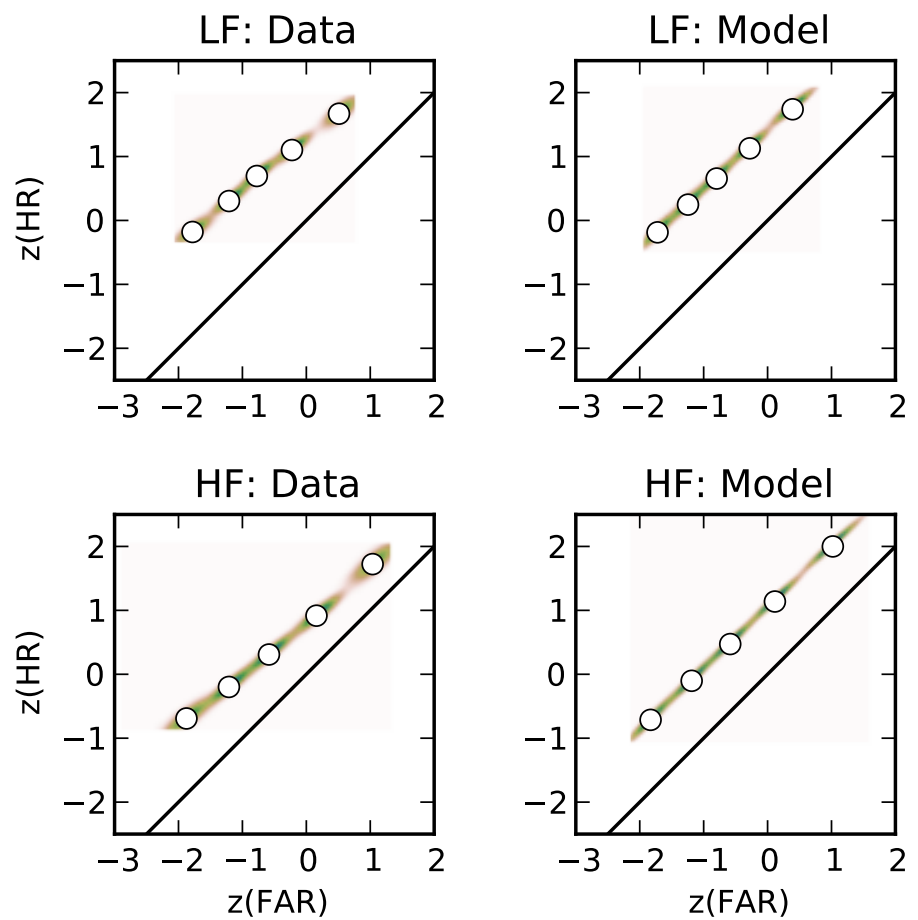

Figure 3.4: Density estimates of the zROC function for both the data of DeCarlo (2007, left) and model (right) for low (top) and high (bottom) frequency words. The circles indicate the median of the z-transformed hit and false alarm rate posterior distributions for each ROC point. 


\section{DeCarlo (2007): Individual Participants}

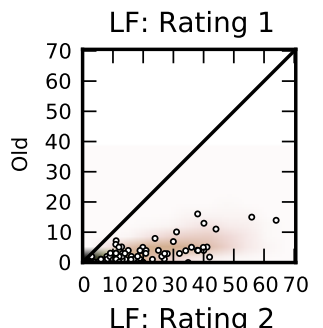

HF: Rating 1

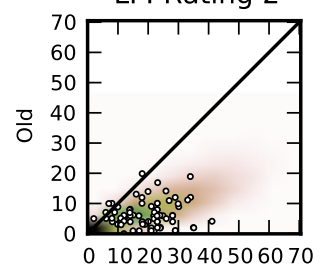

LF: Rating 3
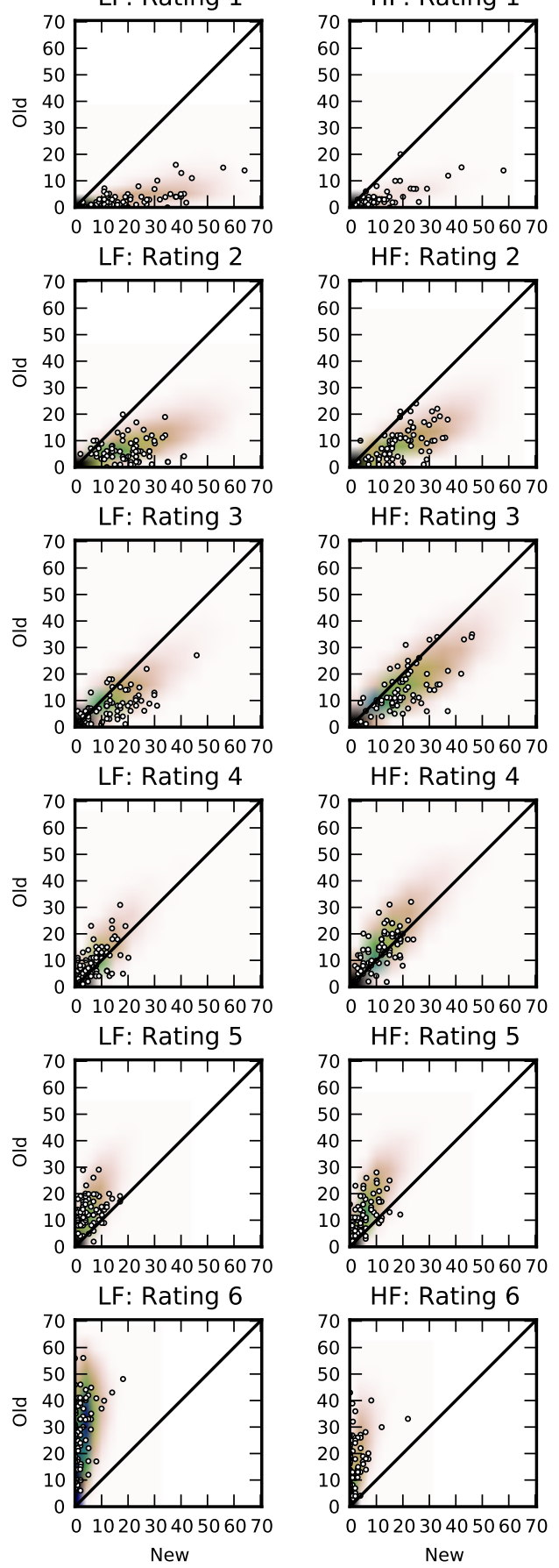

Figure 3.5: Individual participant responses for each confidence rating and word frequency class for the data of DeCarlo (2007). Predicted confidence counts from the model are shown as the colored density estimates. 
context. I allowed for this possibility by allowing a separate set of $\mu_{s s}$ hyperparameters for the long list in the no filler condition. While inspection of Figure 3.1 revealed that the poor contextual reinstatement $\mu_{s s}$ parameter ended up quite high, it was still sufficient to allow the model to predict a small effect of list length in the no filler condition.

Due to the low estimate of the item mismatch variance parameter $\sigma_{t i}^{2}$ for word stimuli, the model predicts virtually no effect of list length in the filler condition. The model also predicts the word frequency mirror effect in the data, predicting both higher hit rates and lower false alarm rates for low frequency words. The performance decrement of delayed testing in the 8 minute filler condition is also addressed by the model, although the magnitude of the performance decrement on both the data and the model's predictions appears to be small.

One should also note that several of the parameters used in the fit to this dataset were constrained across other datasets. The context mismatch variability parameters $\rho$ for low and high frequency words, along with the background noise parameter $\beta$ and item mismatch variability parameter for word stimuli $\sigma_{t i}^{2}$ were also employed in the fit to the data from DeCarlo (2007). The item mismatch variability parameter for word stimuli was also used in the fits to the experiments that employ word pairs, namely the associative recognition experiments that manipulate list length and list strength conducted by Kinnell and Dennis (2012) and Osth and Dennis (2013).

Fits to the individual participant data can be seen in Figure 3.7. One can see that the fit to the individual participants is quite good.

\subsection{List Length and List Strength with Non-Linguistic Stim- uli: Fit to Kinnell and Dennis (2012) and Osth et al. (2014)}

Fits to all of the datasets with non-linguistic stimuli, specifically fractals, scenes, and faces, can be seen in Figure 3.8. This includes the list length experiments conducted by Kinnell and Dennis (2012) and the list strength experiments conducted by Osth et al. (2014). 
Fit to Dennis, Lee, and Kinnell (2008)

No Filler
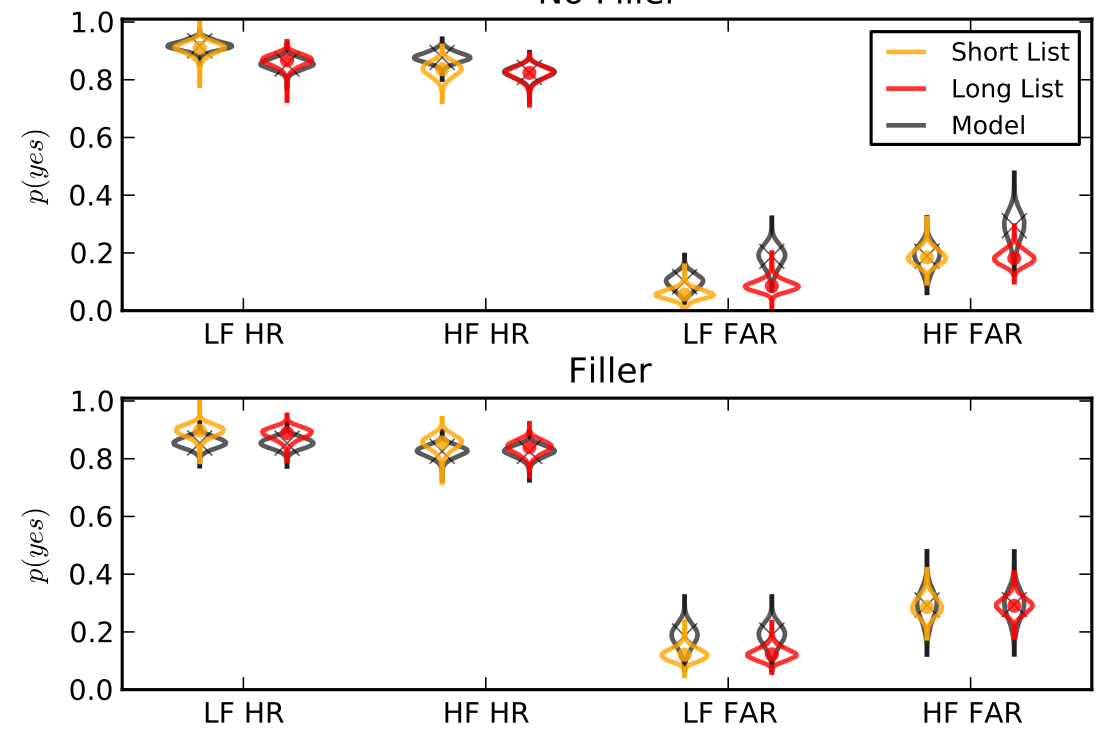

Figure 3.6: Fit to the data of Dennis et al. (2008) for all conditions. Depicted are the density estimates from group level parameters for both the data and the model, along with median posterior estimates for the data (circles) and the model (x's).

\section{Fit to Dennis et al. (2008): Individual Participants}
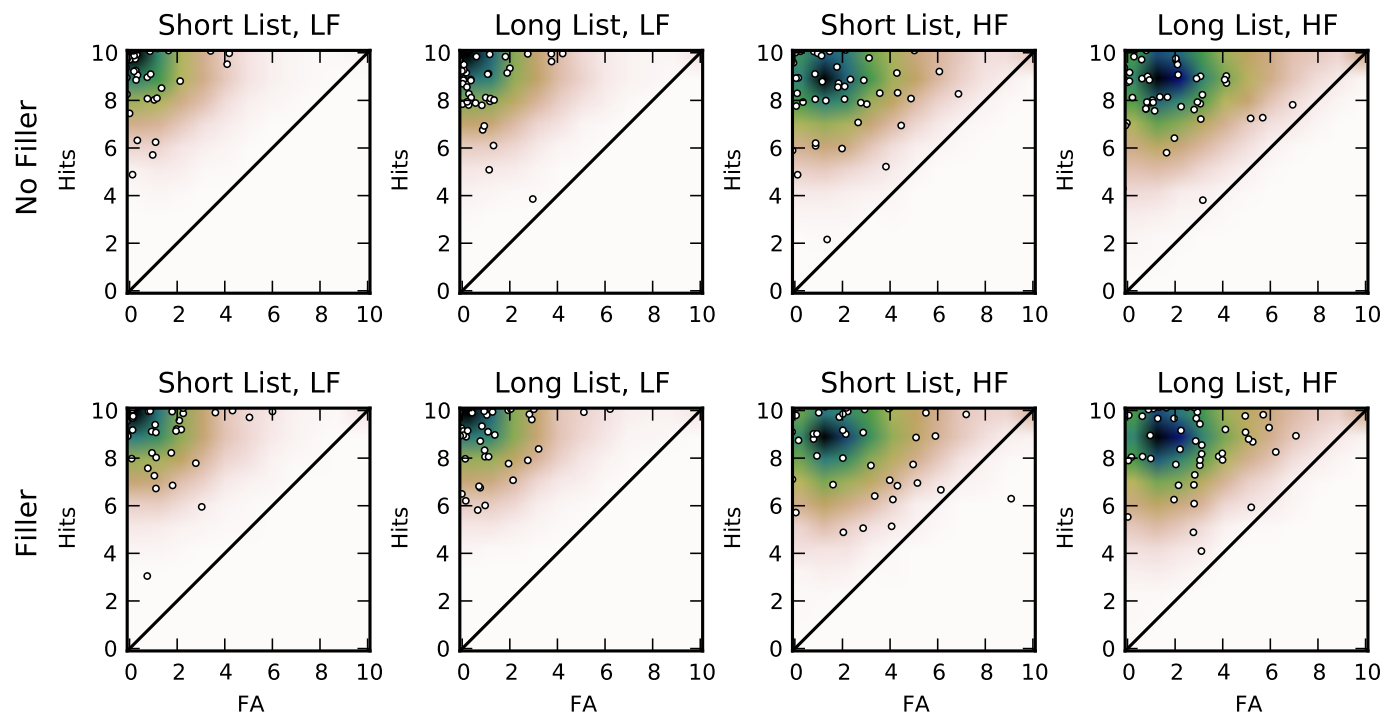

Figure 3.7: Individual participant hit and false alarm counts from the Dennis et al. (2008) dataset for all conditions. Predicted hit and false alarm counts from the model are shown as the colored density estimates. 
The fit to the data is quite good, with the model's predictions falling within the posterior distribution of the data for every comparison. Inspection of the list strength data reveals that the model is adept at predicting the large strength based mirror effect for fractals and for faces, with much lower false alarm rates being predicted for the mixed condition than for the pure weak condition.

The magnitude of the list length and list strength effects is difficult to evaluate on the basis of hit rates and false alarm rates alone. $d^{\prime}$ estimates can be seen in Figure 3.9. For fractals and faces, both the data and the model reveal lower $d^{\prime}$ in the long list and mixed list conditions relative to the short list and pure weak conditions. As was previously mentioned, the detrimental effects of list length and list strength are quite small in both the data and the model's predictions. For scenes, there is no effect of list length or list strength. These predicted effects correspond to the differences in item mismatch variability depicted in Figure 3.1, as list length and list strength effects are predicted for the stimuli with the highest values of item mismatch variability.

Fits to the individual participants for both the list length and list strength paradigms can be seen in Figures 3.10 and 3.11. The fit appears to be quite good.

\subsection{List Length and List Strength with Word Pairs in Asso- ciative Recognition: Fit to Kinnell and Dennis (2012) and Osth and Dennis (2013)}

Fits to the datasets that employ the associative recognition task with word pairs can be seen in Figure 3.12. This includes the list length experiment of Kinnell and Dennis (2012, Experiment 1) and the experiment of Osth and Dennnis (2013) that utilized yes/no responding. The fit to the data is good, with the model predictions falling within the posterior distributions of the data. Nonetheless, the fit to the list length experiment is somewhat worse than the fit to the list strength experiment. This is in part because the performance of the participants appear to be much poorer in the list length experiment than in the list strength experiment despite the similar experimental parameters employed in both experiments. $d^{\prime}$ 


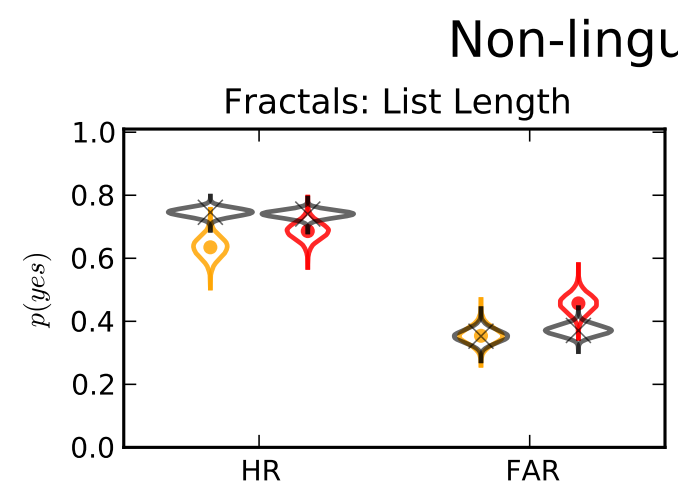

uistic Stimuli
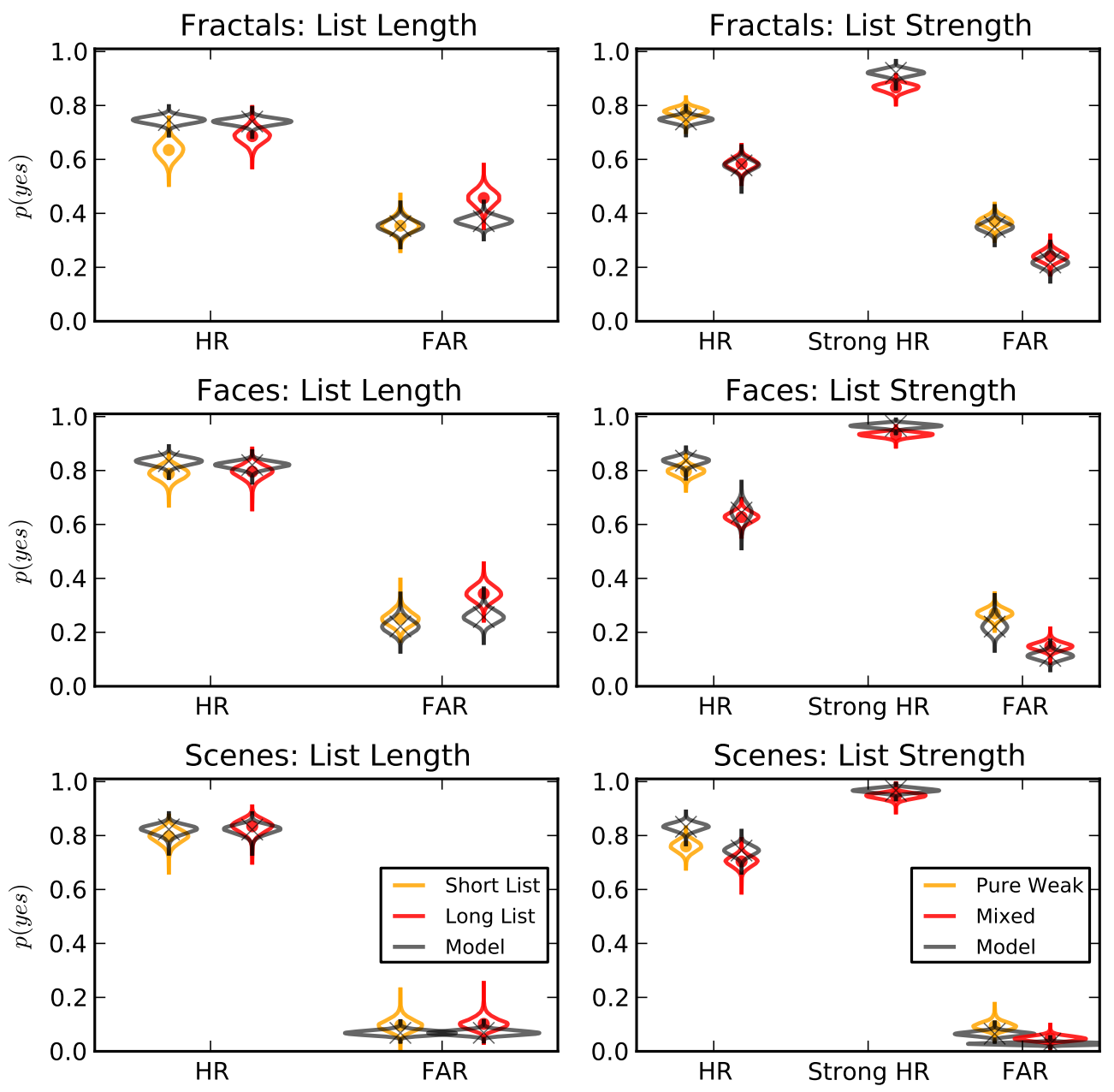

Figure 3.8: Hit and false alarm rates for the data and the model fit to the list length experiments of Kinnell and Dennis (2012, left) and the list strength experiments of Osth et al. (2014, right). Depicted are the density estimates from group level parameters for both the data and the model, along with median posterior estimates for the data (circles) and the model (x's). 


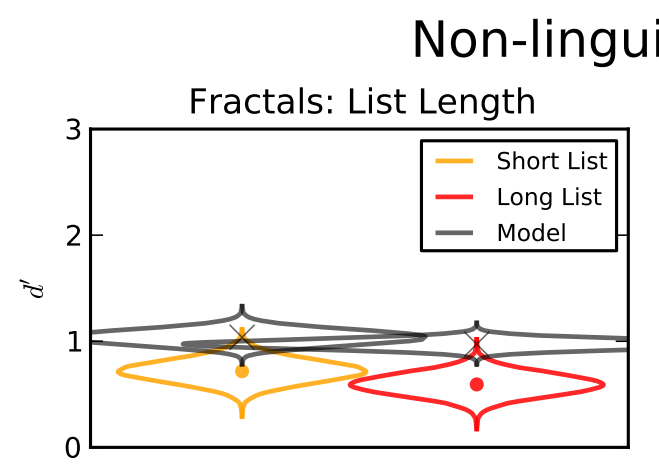

\section{stic Stimuli}
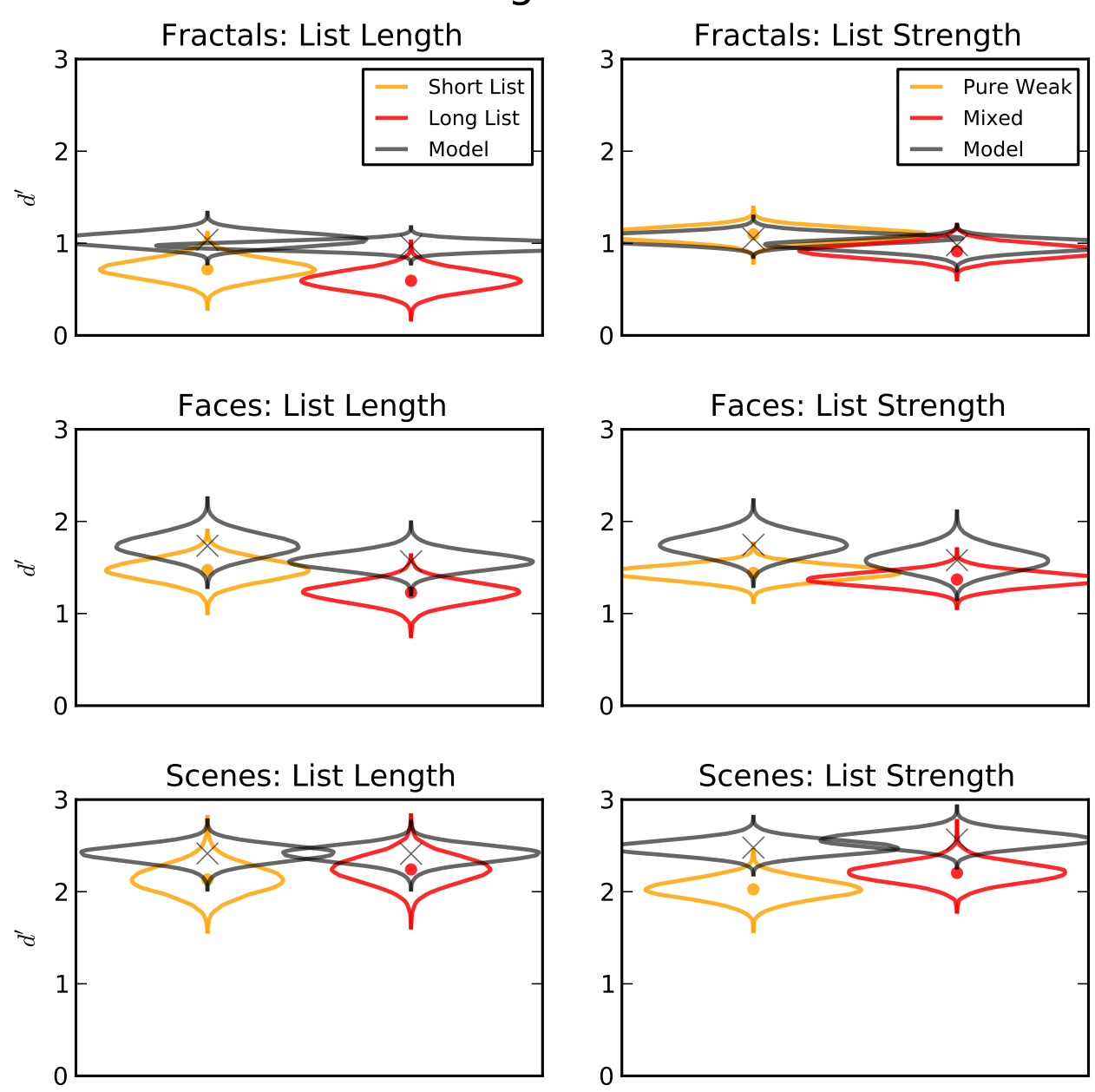

Figure 3.9: Hit and false alarm rates for the data and the model fit to the list length experiments of Kinnell and Dennis (2012, left) and the list strength experiments of Osth et al. (2014, right) that employ non-linguistic stimuli. Depicted are the density estimates from group level parameters for both the data and the model, along with median posterior estimates for the data (circles) and the model (x's). 


\section{Non-linguistic Stimuli Individual Participants}
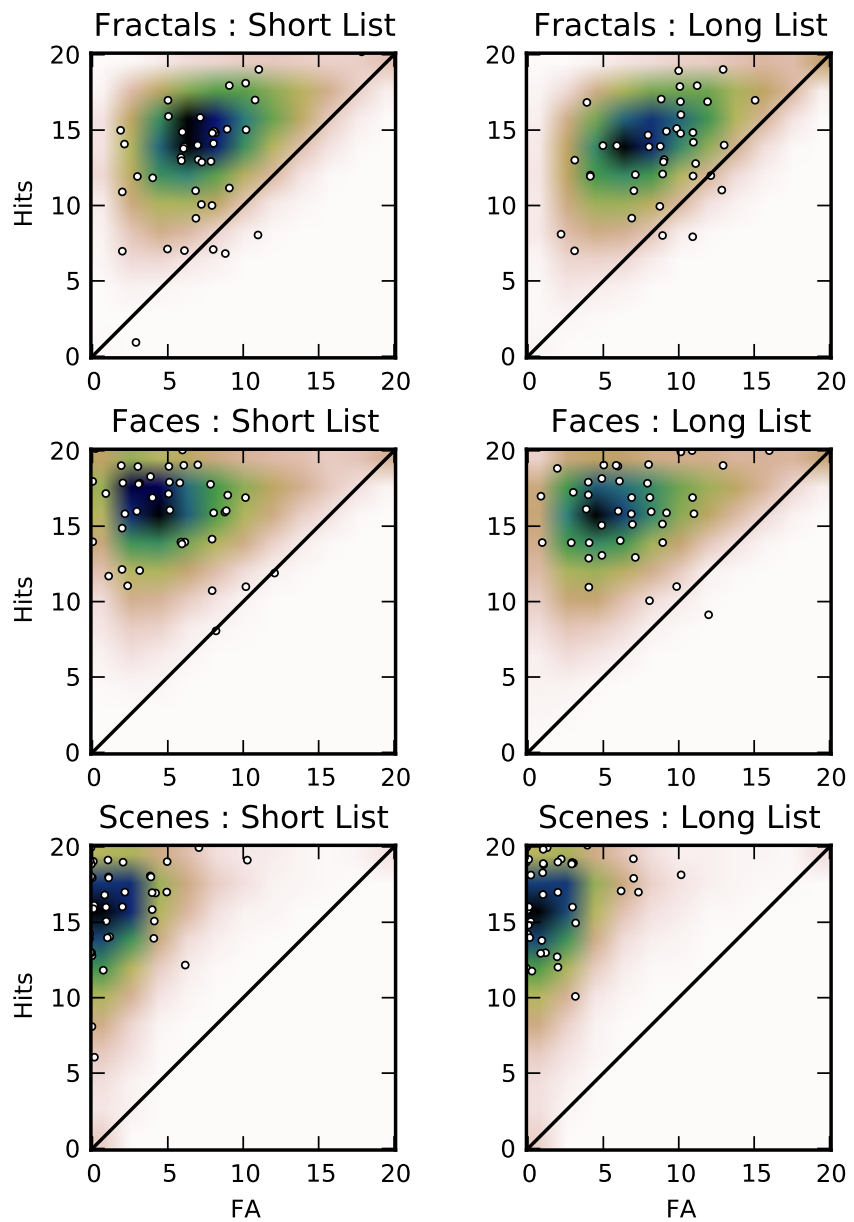

Figure 3.10: Individual participant hit and false alarm counts for the short (left) and long (right) list conditions for fractals (top), faces (middle), and scenes (bottom). Predicted hit and false alarm counts from the model are shown as the colored density estimates. 


\section{Non-linguistic Stimuli Individual Participants}
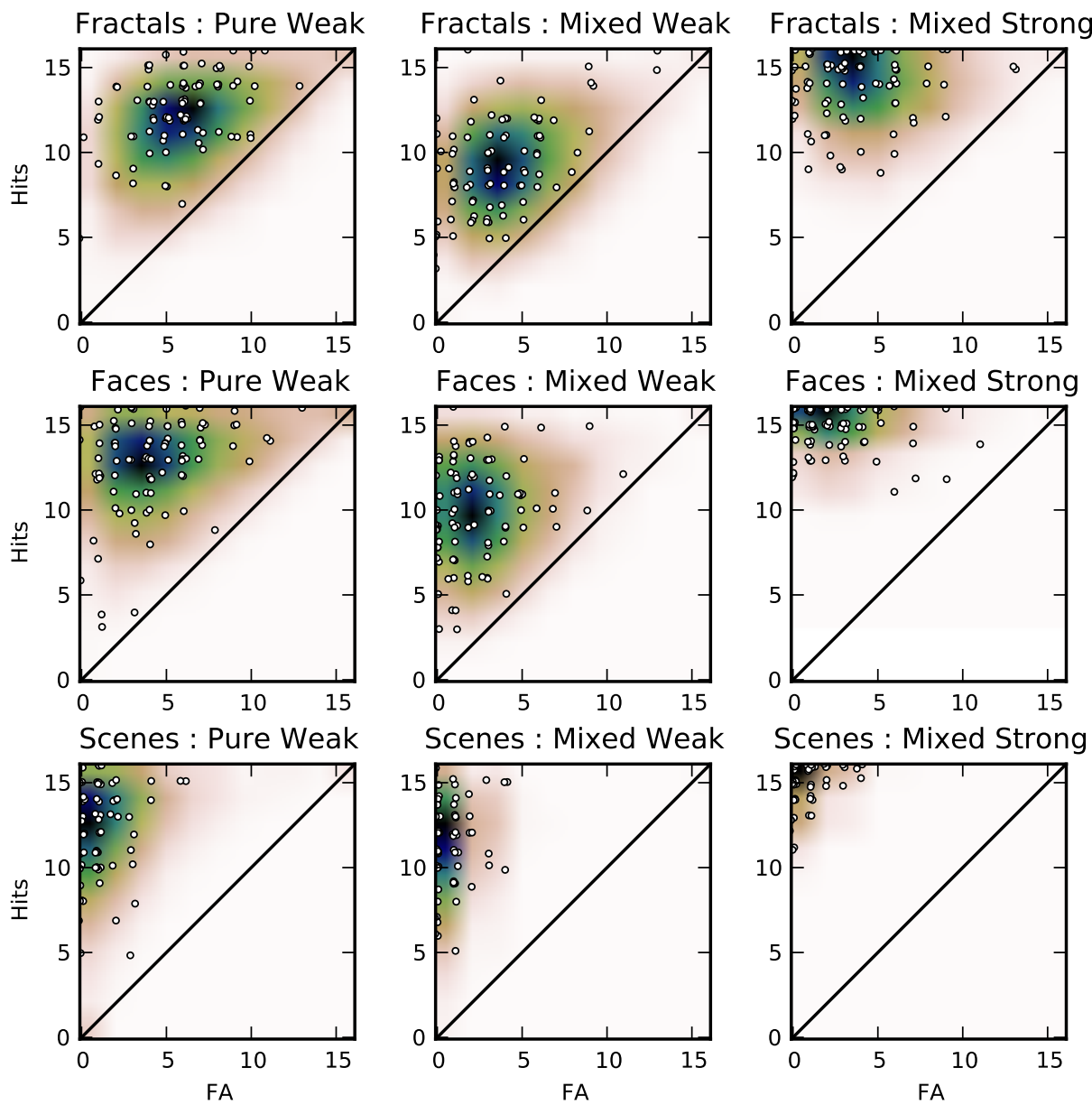

Figure 3.11: Individual participant hit and false alarm counts for the pure weak (left) and mixed weak (middle) and mixed strong (right) conditions for fractals (top), faces (middle), and scenes (bottom). Predicted hit and false alarm counts from the model are shown as the colored density estimates. False alarms are the same for the mixed weak and mixed strong conditions while the hits to $4 \mathrm{X}$ presented items are in the mixed strong plot. 


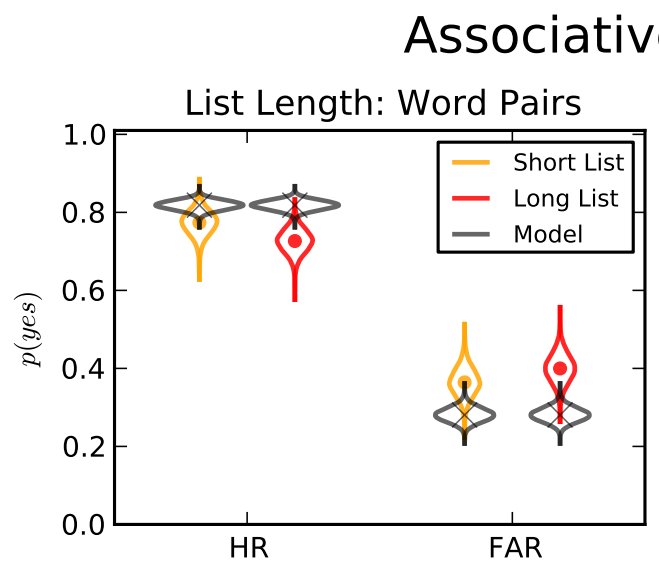

Recognition

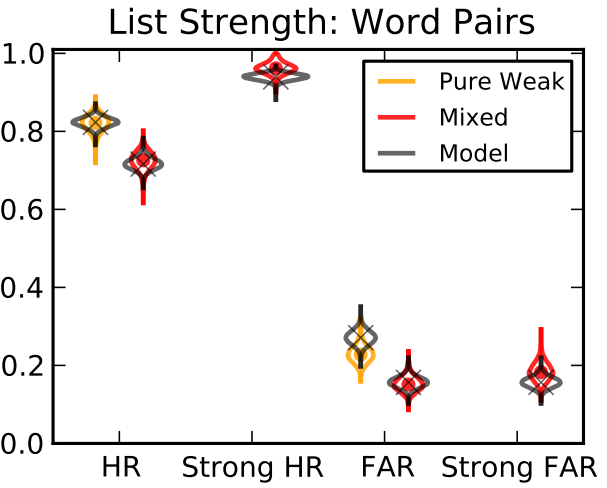

Figure 3.12: Hit and false alarm rates for the data and the model fit to the list length experiments of Kinnell and Dennis (2012, left) and the list strength experiments of Osth and Dennis (2013, right) that employ word pairs in an associative recognition task. Depicted are the density estimates from group level parameters for both the data and the model, along with median posterior estimates for the data (circles) and the model (x's).

estimates can be seen in Figure 3.13. The model predicts no effect of either list length or list strength on associative recognition performance. While performance appears to be somewhat poorer in the long list, this effect was found to not be significant by Kinnell and Dennis (2012) in their analyses.

As mentioned previously, the item mismatch variability parameter $\sigma_{t i}^{2}$ is not just constrained by the manipulations of list length and list strength, but higher values of $\sigma_{t i}^{2}$ predict higher false alarm rates to strong rearraned pairs than weak rearranged pairs in mixed lists. Inspection of Figure 3.12 reveals that the model predicts nearly equivalent false alarm rates between weak and strong rearranged pairs, like in the data. Thus, the model is able to simultaneously predict the cross-list strength based mirror effect (lower FAR in the mixed list than in the pure weak list) as well as the broken within-list strength based mirror effect (weak FAR = strong FAR). This is because the strength estimates used in the likelihood ratio calculation are list-wide: when the strength of a list changes, the strength estimates change. However, on a given test list, the strength estimates are not permitted to change across trials. This is conceptually quite similar to the hypotheses of Stretch and Wixted 


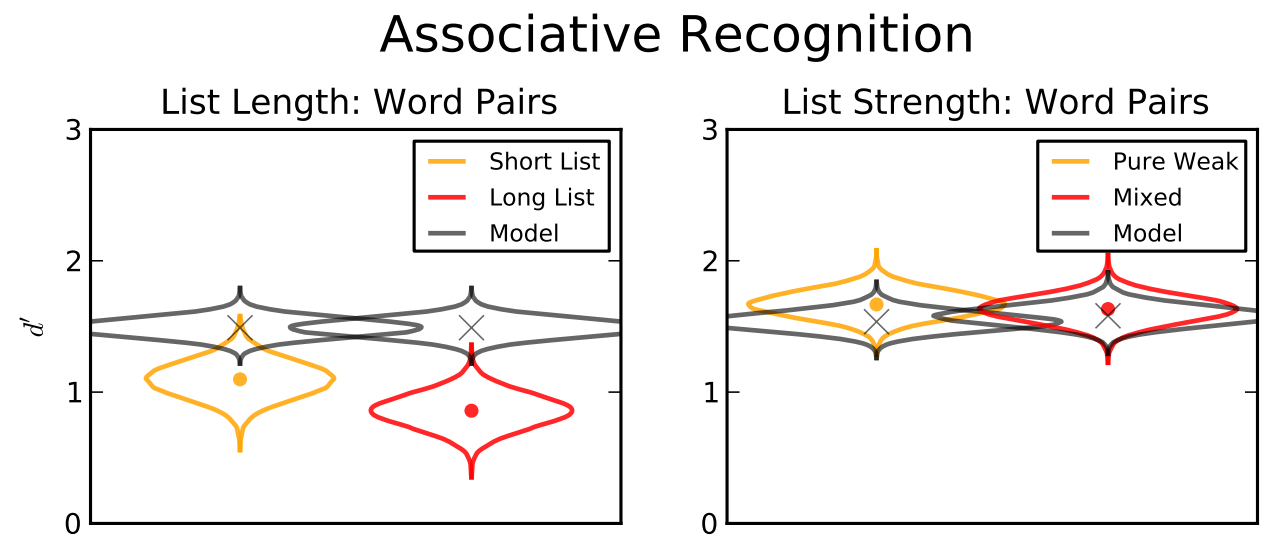

Figure 3.13: Hit and false alarm rates for the data and the model fit to the list length experiments of Kinnell and Dennis (2012, left) and the list strength experiments of Osth and Dennis (2013, right) that employ word pairs in an associative recognition task. Depicted are the density estimates from group level parameters for both the data and the model, along with median posterior estimates for the data (circles) and the model (x's).

(1998b) and Hockley and Niewiadomski (2007), who posited that criterion shifts occur across lists but stay relatively constant within a test list.

Fits to the individual participants for both the list length and list strength paradigms can be seen in Figures 3.10 and 3.11 .

\subsection{Interference Contributions}

To evaluate the contributions from each interference component, the means of the hyperparameters were used to calculate each interference component according to Equations 2.15 and 2.19. Because the variance of the self match is less relevant than the other contributions, only the components of the lure distribution were used in the calculation. For word pairs in associative recognition, the partial match and item noise terms from Equation 2.19 were combined due to the shared influence of the item mismatch variability parameter on their predicted magnitudes. Density estimates of the item noise, context noise, and background noise calculated for each dataset in the fit can be seen in Figure 3.15. Given that context noise was only measured for single word stimuli, context noise is only present in the fits to 

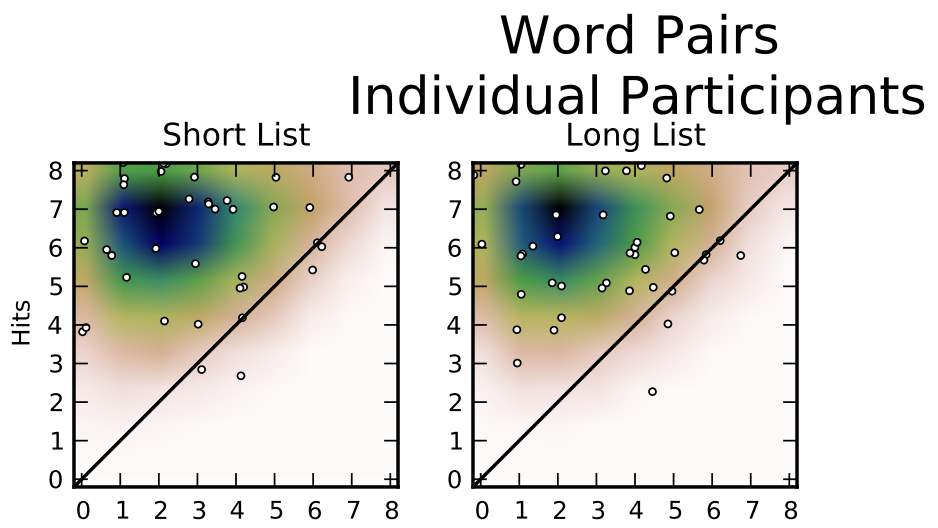

Pure Weak
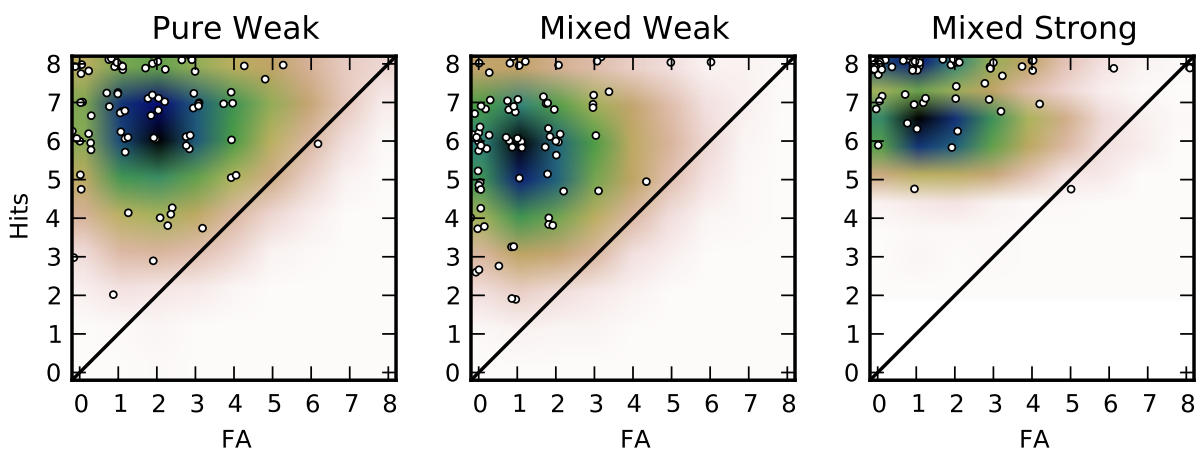

Figure 3.14: Individual participant hit and false alarm counts for list length experiments of Kinnell and Dennis (2012, left) and the list strength experiments of Osth and Dennis (2013, right) that employ word pairs in an associative recognition task. Predicted hit and false alarm counts from the model are shown as the colored density estimates. For the mixed strong condition, hits and false alarm counts are from the strong intact and strong rearranged pairs. 
DeCarlo (2007) and Dennis et al. (2008).

Inspection of Figure 3.15 confirms that the differences in the item mismatch variability parameters across stimulus classes extends to the total item noise as well. Item noise is significantly higher for fractals and faces than for single words, word pairs, and scenes. Additionally, large estimates of background noise can be seen in all of the datasets. Despite the fact that background noise estimates were low for single words, they appear to be quite large relative to the other interference contributions. Item noise, in contrast, does not appear to dominate the other interference contributions in any of the datasets.

To elucidate the relative contributions of each interference component, proportions of total interference were calculated for each interference term. For each dataset, the condition with the highest item noise was used (long lists in the list length experiments and mixed lists in the list strength experiments). Given that the dataset of (DeCarlo, 2007) used shorter lists (70 items) than the longest lists in the (Dennis et al., 2008) dataset (80 items), only the dataset of the Dennis et al. fit was used in this analysis. Additionally, given that only one context noise term contributes at retrieval, separate analyses were performed for the low and high frequency words.

Proportions of total interference can be seen in Figure 3.16, which contains bar plots of the median proportion of total interference for each interference component along with the 95\% highest density interval (HDI). For single words, word pairs, and scenes, item noise is extremely close to zero in its contribution to the total interference. For fractals and scenes, item noise occupies a much greater proportion of the total interference. Nonetheless, for fractals and faces, background noise occupied a significantly greater proportion of interference than item noise in the list length datasets, with $99.6 \%$ and $99.3 \%$ of the background noise minus item noise difference distribution above zero for fractals and faces, respectively. The differences are more ambiguous for the list strength datasets, with $94.4 \%$ and $91.7 \%$ of the background noise minus item noise difference distribution above zero for fractals and faces. For scenes and word pairs, background noise occupies virtually all of the total interference.

Converging evidence was found in an analysis of the interference contributions of the 

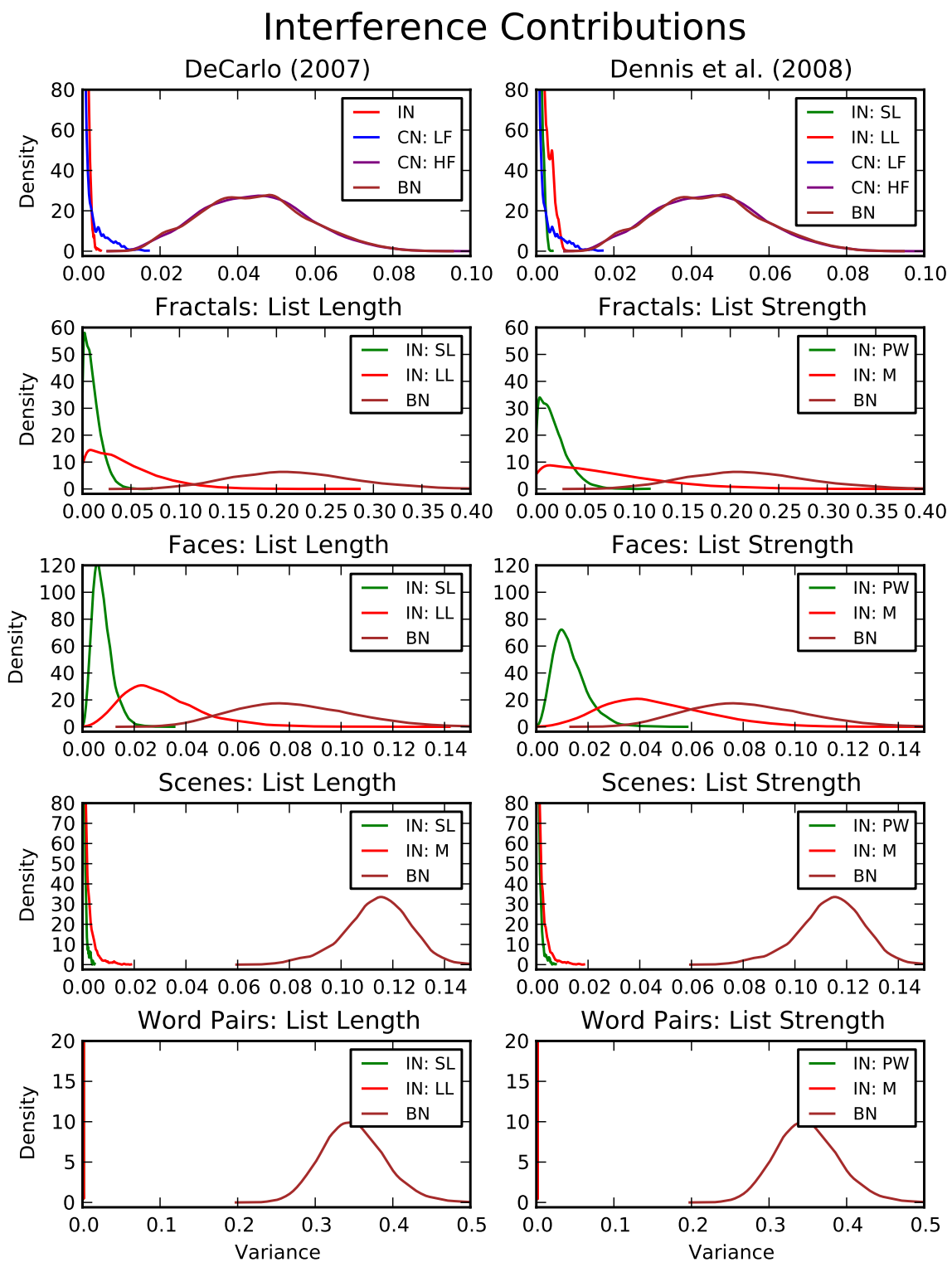

Figure 3.15: Density estimates for item noise (IN), context noise (CN), and background noise $(\mathrm{BN})$ for all datasets. Notes: $\mathrm{LF}=$ low frequency, $\mathrm{HF}=$ high frequency, $\mathrm{SL}=$ short list, $\mathrm{LL}=$ long list, $\mathrm{PW}=$ pure weak, and $\mathrm{M}=$ mixed. 


\section{Proportions of Total Interference}
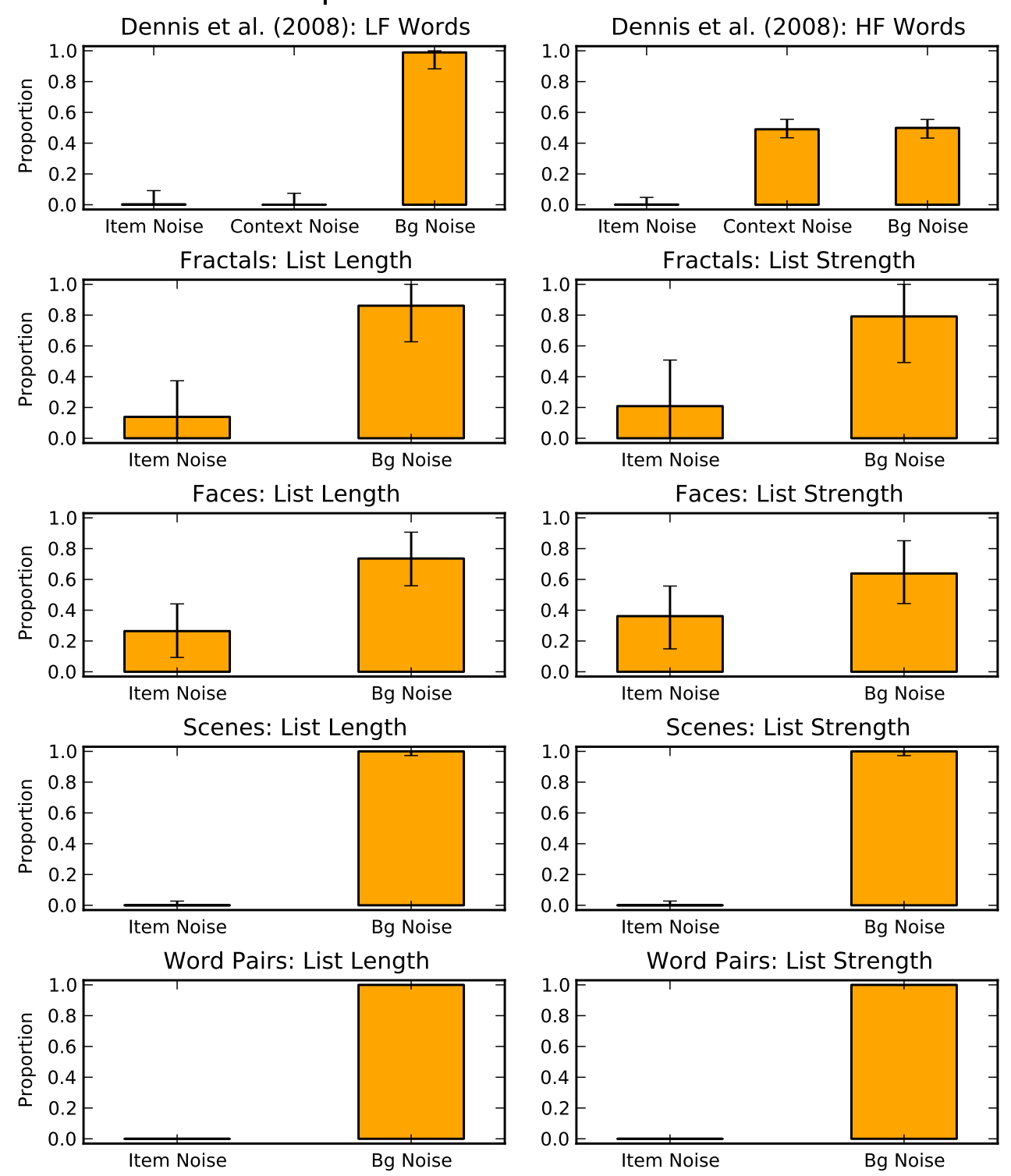

Figure 3.16: Median proportions of total interference for item noise, context noise, and background noise (bg. noise) for the datasets of Dennis et al. (2008), Kinnell and Dennis (2012), Osth et al. (2014), and Osth and Dennis (2013). Error bars represent the 95\% highest density interval (HDI). Notes: $\mathrm{LF}=$ low frequency, $\mathrm{HF}=$ high frequency. 
individual participants. For each participant, difference distributions were constructed between the item noise of the highest item noise condition and the other interference contributions. Following statistical conventions, differences among the interference contributions were deemed significant if $95 \%$ of the area of the difference distribution lied above zero. For words in the Dennis et al. dataset, all of the participants exhibited significantly higher background noise than item noise and significantly higher context noise for high frequency words than item noise. For word pairs, all of the participants exhibited higher background noise than item noise in both the list length and list strength datasets. For scenes, 97.5\% of the participants exhibited higher background noise than item noise in the list length dataset, whereas all of the parcicipants exhibited higher background noise than item noise in the list strength dataset. For fractals and faces, none of the participants exhibited a dominant interference contribution.

One of the surprising findings of this analysis is that context noise is virtually zero for low frequency words (median of .03\% of total interference), with background noise occupying nearly all of the interference (median: 98.7\%). For high frequency words, roughly equivalent levels of context noise and background noise are present. How could low frequency words exhibit such little context noise? In the fits, I collapsed across the number of occurrences of a word $(m)$ and the variability in the similarity to previous contexts $\left(\sigma_{s u}^{2}\right)$. One possibility is that contexts are quite dissimilar to previous contexts, meaning that the true value of $\sigma_{s u}^{2}$ is quite low. High frequency words may suffer from considerable interference not because of the overlap among contexts, but due to their frequent exposures (a high value of $m$ ). 


\section{Chapter 4 General Discussion}

When cues are globally matched against the contents of memory the output of the retrieval process can be characterized on the basis of matches and mismatches to the item and context cues employed at retrieval. I fit a global memory model based on the tensor model of Humphreys, Bain, and Pike (1989) that directly parameterizes the matches and mismatches to the item and context cues to ten recognition memory datasets to obtain estimates of item noise, context noise, and background noise. The model parameters were constrained by the manipulations of strength, list length, list strength, word frequency, study-test delay, and stimulus class. Maximum constraint was imposed on the model by constraining several of the model's parameters across multiple datasets.

Resulting parameter estimates derived from a hierarchical Bayesian analysis revealed that item noise plays a rather small role in retrieval, although the magnitude of its influence depends on the stimulus class. For words, word pairs, and scenes, item noise is extremely close to zero and interference stems entirely from pre-experimental sources, namely context noise and background noise. For fractals and faces, item noise is much larger, although background noise appears to be the dominant source of interference for these stimulus classes in some comparisons (the list length datasets). None of the analyses revealed a dominant influence of item noise in any of the stimulus classes or individual participants. These results were particularly surprising because the contributions of background noise have generally been ignored by models of episodic recognition. In the following sections, I discuss the implications for these parameter estimates in the recognition memory literature, such as 
what the parameter estimates imply about the underlying representations of linguistic and non-linguistic stimuli and comparisons to previous investigations which have argued for higher magnitudes of item noise.

\subsection{Plausibility of Low Item Noise}

While the results of the model fit demonstrate that item noise makes a relatively small contribution to the total interference in recognition memory tasks, it does not specify what the vector representations of the items are that would result in low item noise. It has been argued that no interference among the items can be exhibited when item representations are orthogonal to each other (Dennis \& Humphreys, 2001; Osth \& Dennis, 2013). At a psychological level, this would imply that item representations are dissimilar and share no features with each other. The results of the fitting imply that there is a non-zero contribution of item noise at retrieval, which rejects the notion of orthogonal item representations. Nonetheless, a close approximation would be employing relatively sparse item representations, meaning that they are not completely orthogonal but exhibit minimal overlap with each other.

Some might find such an idea implausible, especially given that words in recognition memory tasks are often perceived as similar to each other in meaning, phonology, and surface form. How then could their representations be dissimilar? A number of theories of hippocampal function describe the function of the hippocampus as creating sparse representations from overlapping inputs (Kumaran \& McClelland, 2012; Marr, 1971; McClelland, McNaughton, \& O'Reilly, 1995; Treves \& Rolls, 1992; Norman \& O'Reilly, 2003; O'Reilly \& McClelland, 1994; O'Reilly \& Rudy, 2001), allowing the hippocampus to exhibit fast learning, discriminate among highly similar novel inputs, and minimize interference, all qualities that are critical for performance on episodic memory tasks. Tasks which require the similarity among the items to be emphasized can employ the distributed representations in the neocortex that have been developed from experience, which is a central component of the complementary learning systems (CLS) theory (McClelland et al., 1995). 
Another possible interpretation is that item representations are multidimensional and that their dimensions can be weighted based on their relevance to a cognitive task, as proposed by the generalized context model (GCM: Nosofsky, 1986, 1991). In this regard, dimensions among the items in a recognition memory task that emphasize their similarity, such as their shared semantics or surface appearance, may be de-weighted for optimal discrimination of old from new items. This approach yields similar interpretations as to the hippocampal theories, in that representations of items are not fixed, but adapt to the task faced by the participant. Thus, the results of the model fitting do not imply that word representations are dissimilar in all cognitive tasks, merely that they are dissimilar in episodic memory.

Another possible objection to the finding of minimal item noise in recognition memory is that in the free recall task, manipulations of list length (Murdock, 1962; Roberts, 1972; Ward, 2002) and list strength (Malmberg \& Shiffrin, 2005; Ratcliff et al., 1990; Tulving \& Hastie, 1972) exhibit robust decrements on recall performance. How could a memory system which exhibits minimal interference among the items in recognition memory exhibit such strong competition among the items in a free recall task? As it turns out, the majority of current free recall models exhibit competition among the items not because of overlap in their item representations, but due to usage of a sampling with replacement memory search process (Davelaar, 2007).

In resampling models, the context cue initiates the probabilistic sampling of items that are most strongly bound to the list context and sampled items are recalled if they exceed a specified threshold. On subsequent recall attempts, previously recalled items are not removed from the set of recall candidates. Instead, they can continue to be sampled by the search process instead of items that have not yet been recalled. List length effects fall naturally from resampling models as longer lists contain more candidates to be output, decreasing the probability that any particular item will be sampled. Davelaar (2007) has demonstrated that resampling allows for the prediction of both list length effects and the increase of inter-response times as recall proceeds (Murdock \& Okada, 1970). Additionally, other current models of free recall with specified item and context representations, 
such as the model of Davelaar et al. (2005) and the temporal context model (Howard \& Kahana, 2002; Sederberg et al., 2008), employ orthogonal item representations and predict detrimental effects of list length on free recall performance.

While these models have not simulated the list strength paradigm, resampling provides an intuitive explanation: increasing the strength of a subset of list items increases their sampling probability and decreases the sampling probability of the non-strengthened items. Resampling also provides a similar and intuitive explanation of the finding of output interference in recall tasks (Dalezman, 1976; Dong, 1972; Roediger, 1974; Roediger \& Schmidt, 1980). Across a number of experiments, it has been demonstrated that recalling a subset of the list items decreases the recall probability of the remaining items. If it is assumed that the act of recall strengthens the recalled items (as was assumed by the SAM model, Raaijmakers \& Shiffrin, 1981), then the sampling probability of the recalled items will increase at the expense of the non-recalled items, producing output interference as a consequence.

Thus, while it is clear that the free recall task exhibits evidence for competition among the list items at retrieval, the success of free recall models in addressing effects of list length, list strength, and output interference comes from the usage of resampling during memory search. These effects do not necessitate inter-item similarity among the list items and a model such as ours that exhibits minimal item noise in recognition memory could be capable of exhibiting competition in a free recall task through the usage of resampling at retrieval during a free recall task.

\subsection{Arguments for Item Noise Models}

There have been a number of experimental findings in the recognition memory literature that have been used to argue for a larger contribution of item noise than I have estimated in my model fits, specifically the effects of manipulations of semantic similarity and decrements in performance that occur through recognition testing. Here, I discuss these arguments in detail and describe how they are in fact compatible with the results and interpretations from my modeling work. 


\subsubsection{Effects of Semantic Similarity on Recognition Memory}

One line of evidence that has been used to argue for the idea that item noise plays a substantial role in recognition memory is the finding that increasing the number of semantically similar words on a study list decreases performance. Typically, this is accomplished in recognition experiments by increasing the number of studied items from the same semantic category, and decreases in performance with increasing category length are often observed (e.g.: Arndt \& Hirshman, 1998; Criss \& Shiffrin, 2004; Dewhurst \& Anderson, 1999; Shiffrin et al., 1995; Zaki \& Nosofsky, 2001). Global matching models predict that as more similar items are entered into memory, it becomes increasingly difficult to discriminate between studied and unstudied exemplars from the same category.

While the present model fits demonstrate that the magnitude of the item noise contribution for words is quite small, I have fit data from experiments that employed lists of unrelated words. As I have previously mentioned, the magnitude of item noise is dependent on the stimulus class. Thus, one possibility is that unrelated words exhibit very low inter-item similarity whereas words that share a semantic category are sufficiently similar to generate more substantial degrees of item noise. This approach was undertaken by Johns et al. (2012) who used a holographic memory model where the item representations are high dimensional vectors generated from the TASA corpus (Landauer \& Dumais, 1997). Specifically, each dimension in the vector reflected a particular document in the corpus and the dimension took a value of one if the word occurred in that document and a zero otherwise. The resulting vectors were quite sparse, and consequently there was virtually no overlap among the vectors of unrelated words and no effects of list length and list strength were predicted. However, similar words overlap quite substantially due to their co-occurrence in documents among the corpus, and for that reason the model was able to predict higher false alarm rates for semantically related lures in the Deese-Rooediger-McDermott paradigm (DRM: Deese, 1959; Roediger \& McDermott, 1995).

However, there are a number of complications from category length designs that prevent me from endorsing the view that semantically similar words exhibit high degrees of item 
noise. Specifically, there are a number of lines of evidence that suggest that other factors complicate the interpretation of category length effects. Changes in performance across category length do not appear to be purely a consequence of having studied similar content, but also appear to reflect changes in decision criteria and usage of category labels as cues to guide retrieval.

Pure item noise models predict that as category length is increased, performance should decrease monotonically, as the higher number of similar studied items in memory makes it more difficult to discriminate between studied items and highly similar lures. However, Neely and Tse (2009) found that the change in performance is actually non-monotonic, with performance increasing as category length increased from 1 to 2 items, decreasing to the baseline level as category length increased to eight, and further decreasing as category length increased to fourteen items. Neely and Tse (2009) suggested that the increase in performance could be achieved if additional category labels are used at retrieval, allowing memory to be more focused on items from a studied category if the category length is relatively small (such as when category length is 2 items).

When category labels get used as cues along with the studied items, the task begins to resemble an associative recognition task, as the question being asked by the participant during a recognition test is no longer "Did I see this item on the study list?" but "Did I see this item in one of the categories I studied?" 8 Such a view predicts that increases in category length, which would increase the likelihood that category labels would be used as cues, should produce an increase in the ability to discriminate studied categories from unstudied categories. Dennis and Chapman (2010) found exactly this pattern. They conducted a category length experiment where category length and list length were manipulated by presenting eight categories with category lengths of one, three, and ten exemplars. Since the list length increased as the category length is increased, a pure item noise model predicts that discrimination of studied from unstudied categories should get worse with increasing length. Instead, false alarm rates to exemplars from unstudied categories decreased as category length was increased. Fits of the REM model found that the model predicted an

${ }^{8}$ We would like to acknowledge Michael Humphreys for conceiving of this analogy. 
increase in the false alarm rate to exemplars from unstudied categories and that the model was not able to fit the data even when the criteria were allowed to vary across the category length conditions. The data were able to be fit with BCDMEM, which exhibits no item noise, by assuming that category length increases the likelihood that category labels are used as cues in conjunction with the probe at retrieval.

Another prediction from pure item noise accounts of the category length effect is the within-category choice advantage on forced choice tests (Clark \& Gronlund, 1996; Clark, 1997; Hintzman, 1988). A counter-intuitive prediction of the models is that on a $2 \mathrm{AFC}$ recognition test, recognition should be superior when both choices are from the same category than when both choices are from different categories. The models make this prediction because when two similar items are presented, the memory strengths of the two items are correlated. When the difference between the two memory strengths is calculated, the covariance between the two similar items gets subtracted out, resulting in a difference distribution that has lower variance for within-category choices than for between-category choices. While Hintzman (1988) found confirmatory evidence for this prediction, Maguire, Humphreys, Dennis, and Lee (2010) noted that Hintzman compared between and within category choices as a between subjects manipulation, which may have changed the way participants approached the test. Additionally, participants were tested using booklets, which did not properly control for lag between presentations, the order of testing, etc. In the experiments of Maguire et al., categories were generated from either word association norms or taxonomically generated categories and the authors found no within-category choice advantage in all experimental conditions. They additionally confirmed the null hypothesis by using the Bayesian analysis developed by Dennis et al. (2008).

While a number of these experiments have found aspects of the data that were contrary to the predictions of the item noise account, both Dennis and Chapman (2010) and Maguire et al. (2010) found increases in the false alarm rate to unstudied exemplars from studied categories with increases in category length that were greater than the corresponding increase in the hit rate, producing a net decrease in $d^{\prime}$. However, one difficulty with usage of $d^{\prime}$ is its assumption of an equal variance signal detection model, which is almost 
uniformly violated in ROC experiments. If an equal variance model is used in the analysis when unequal variance is the true model, changes in the response criterion can additionally change $d^{\prime}$ (Rotello, Masson, \& Verde, 2008) and for that reason, $d_{a}$ is often recommended for analysis. Cho and Neely (2013) conducted a category length experiment where they employed category lengths of two, eight, and fourteen exemplars while collecting confidence ratings in yes/no testing and employed $2 \mathrm{AFC}$ testing. They additionally insured that for all category lengths the same number of items were tested in each category and that all items came from the same serial position in the category. While category length increased hit rates and false alarm rates and decreased $d^{\prime}$, there was no effect of category length on $d_{a}$, suggesting that a criterion shift may have been taking place ${ }^{9}$. Additionally, there was no effect of category length on $2 \mathrm{AFC}$ recognition performance and no within-category choice advantage in the 2AFC tests, replicating the results of Maguire et al. (2010).

The lack of decrement in performance with increasing category length, the lack of withincategory choice advantage, and the better discrimination of studied categories from unstudied categories with increasing category length are contrary to the the pure item noise account of category length effects. However, I would like to state explicitly that these results do not preclude the idea that item representations for semantically similar items exhibit more similarity to each other than unrelated words, making them more susceptible to item noise. In fact, this idea is highly plausible. Instead, these results indicate that other cognitive factors appear to be contributing to the observation of category length effects with categories constructed from word stimuli, and these factors complicate making inferences about the differing susceptibility to item noise across differring degrees of semantic similarity of the stimuli. The higher similarity among the item representations for similar words may be so negligibly small that category length would have to be manipulated to much higher numbers than in conventional experiments to observe effects consistent with the item noise account, such as the within-category choice advantage.

\footnotetext{
${ }^{9}$ An alternative possibility is that manipulations of category length increase the mean of the target and lure distributions. While item noise accounts make this prediction, they further predict that the variance of both distributions should increase and performance should decrease as a consequence. Nonetheless, usage of a category label as an additional cue may have the effect of shifting both distributions upward without decreasing discriminability.
} 
Additionally, I have restricted this discussion to experiments that have employed words as stimuli, as that accounts for the majority of the category length experiments that have been conducted. My modeling results indicate that certain non-linguistic stimuli may be more susceptible to item noise than word stimuli, indicating that category length effects consistent with the item noise account should be detectable using non-linguistic stimuli. Consistent with this idea, Konkle, Brady, Alvarez, and Oliva (2010) found robust category length effects in 2AFC testing using photos of objects. Furthermore, they employed many of the same controls employed by Cho and Neely (2013)'s investigation which failed to find category length effects with words, such as always testing the same number of items for each category length and testing the same serial positions within each category.

\subsubsection{Decrements in Performance Through Recognition Testing}

One of the more recent lines of research that has been used to argue for models where item noise is the bulk of interference in recognition memory concerns the decrease in performance across test trials in a recognition memory test, which has been robustly observed in many experiments (Annis, Malmberg, Criss, \& Shiffrin, 2013; Criss et al., 2011; Gillund \& Shiffrin, 1984; Kim \& Glanzer, 1995; Malmberg, Criss, Gangwani, \& Shiffrin, 2012; Murdock \& Anderson, 1975; Peixotto, 1947; Ratcliff \& Hockley, 1980; Ratcliff \& Murdock, 1976; Schulman, 1974). While this finding had been known for some time, it was unclear whether the observed decrease was due to the experience of the test items or the increase in retention interval due to the passage of time. Recently, Criss et al. (2011) argued that the decrease in performance was purely due to accumulated item noise, as one of their experiments (Experiment 2) included a condition where recognition testing was delayed by a 20 minute filler task. The 20 minute delay exhibited only a relatively small decrement to performance and was much smaller than the decrement that was observed through the course of recognition testing, thus ruling out the hypothesis that the testing decrement is due to the passage of time. Converging evidence for the argument that the testing decrement reflects item noise comes from Murdock and Anderson (1975), who found that the magnitude of the testing decrement increased with the number of choices on a forced choice recognition test. If all 
items on each forced choice trial are added to the contents of memory, then it naturally follows that trials with more choices should exhibit greater performance decrements as a consequence of the higher item noise.

Possibly the biggest challenge to the item noise hypothesis of the testing decrement is that it is unable to explain the fact that the number of test items exhibit much larger decrements on performance than the number of study items, which typically exhibit no decrement at all under controlled conditions. For instance, Schulman (1974) compared list lengths of 25, 50, and 100 items with equated retention intervals across each list length condition and compared performance across blocks of 25 AFC trials. For each test block, performance was equivalent across the different list length conditions and yet there were considerable decrements in performance across the test blocks. A pure item noise account predicts poorer performance both in later test blocks and for larger list length conditions.

A plausible contender to the item-noise hypothesis is that contextual drift through the course of testing impairs performance. That is, each test trial may alter the context cue, which decreases the match to the studied items and consequently decreases recognition performance. The majority of contextual drift theories assume that items, not the passage of time, are the sources of contextual change (Mensink \& Raaijmakers, 1988; Murdock, 1997; Howard \& Kahana, 2002). Thus, Murdock and Anderson's (1975) observation that more choices on a forced choice trial cause greater decrements in performance can be understood as greater contextual change as a consequence of experiencing more items on each trial. While one could argue that the contextual drift explanation is ad hoc, several investigators have independently posited the idea that retrieval from episodic memory causes contextual change (Jang \& Huber, 2008; Klein et al., 2007; Sahakyan \& Hendricks, 2012). In Jang and Huber's investigation, they found that retrieval during episodic tasks produced greater contextual change than other forms of retrieval, which can explain why testing produced far greater decrements in recognition memory performance in Criss et al.'s (2011) investigation than that of a 20 minute retention interval.

To demonstrate that the contextual drift account can reasonably account for the testing decrement, I simulated the paradigms of Schulman (1974) and Murdock and Anderson 

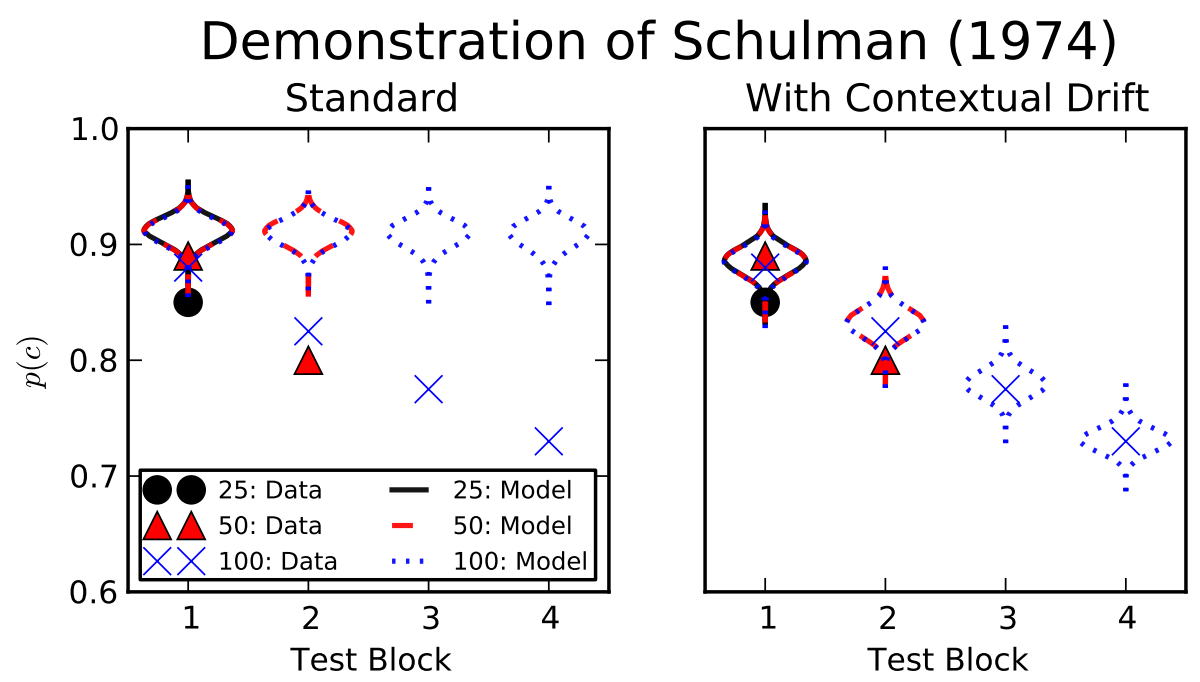

Figure 4.1: Group level predictions for the Schulman (1974) paradigm using parameters derived from the model fit, where on each individual trial two items are added to the contents of memory. The left panel shows the model predictions without parameter modification, while the right panel shows the model's predictions where each item on a test trial multiplies the mean context match by .9955. Due to an inavailability of the original data from the Schulman (1974) dataset, the data means were approximated from the graphs in the original article.

(1975) with the model. Group-level predictions from the model for the dataset of Schulman (1974) were derived by using hyperparameters that most closely resembled the experimental parameters of the Schulman experiment. Due to the usage of high frequency word stimuli, a short study-test delay, and a two second study time, I used the context mismatch variability parameter for high frequency words, the mean context match for the three and a half minute delay, the item mismatch variability parameter for word stimuli, and the learning rate for the three second study time (which had to be multiplied by .9 to get better resemblance to performance). All individual trials were simulated, and after each trial, two items were added to the contents of memory that corresponded to the two choices on each test trial. The mean context match for test items was set to one to reflect the greater recency for test items over study items.

The model predictions can be seen in the left panel of Figure 4.1. One can see that the model correctly predicts no decrement of increasing list length (the data show better per- 
formance in longer lists, although the differences were not significant), but fails to produce levels of item noise that are sufficient to produce a testing decrement as large as what is seen in the data. I simulated the contextual drift assumption by assuming that for each item on each test trial, the mean match to context for study and test items was multiplied by .9955 to reflect contextual drift caused by recognition testing. Predictions from the contextual drift assumption can be seen in the right panel of Figure 4.1, and one can see that it succeeds in capturing both critical aspects of the data: no effect of list length on recognition performance is predicted, while performance decreases dramatically with each block of recognition testing.

Group-level predictions for the dataset of Murdock and Anderson (1975) can be seen in Figure 4.2. Given that there was immediate testing in their experiment, the mean match to context parameter $\mu_{s s}$ was set to one. The study time and word frequency for the experiment was not specified, although I obtained reasonable correspondence with the data using the learning rate $r$ from the three second study time and the context mismatch variability $\rho$ for high frequency words. The left panel shows the predictions from the model when no contextual drift is employed and each test item is added to the contents of memory. One can see that while performance is worse as the number of choices is increased, the level of item noise is insufficient to produce any significant decrements across testing. The right panel shows the performance of the model with the additional assumption that each item on a test trial multiplies the mean match to context by .9985 . One can see that the model correctly predicts a larger decrease as the number of choices on each forced choice trial was increased.

A more recent result that has been used to argue for an item noise interpretation of the testing decrement comes from an investigation that used blocked categories at test. Malmberg et al. (2012) conducted an experiment where all studied words came from two semantic categories and included a blocked condition where the test list was divided into two blocks of 150 2AFC trials where each block tested a different semantic category. Performance decreased monotonically through the test list until the category switch point, at which point performance increased considerably and subsequently decreased over further 


\section{Demonstration of Murdock and Anderson (1975)}
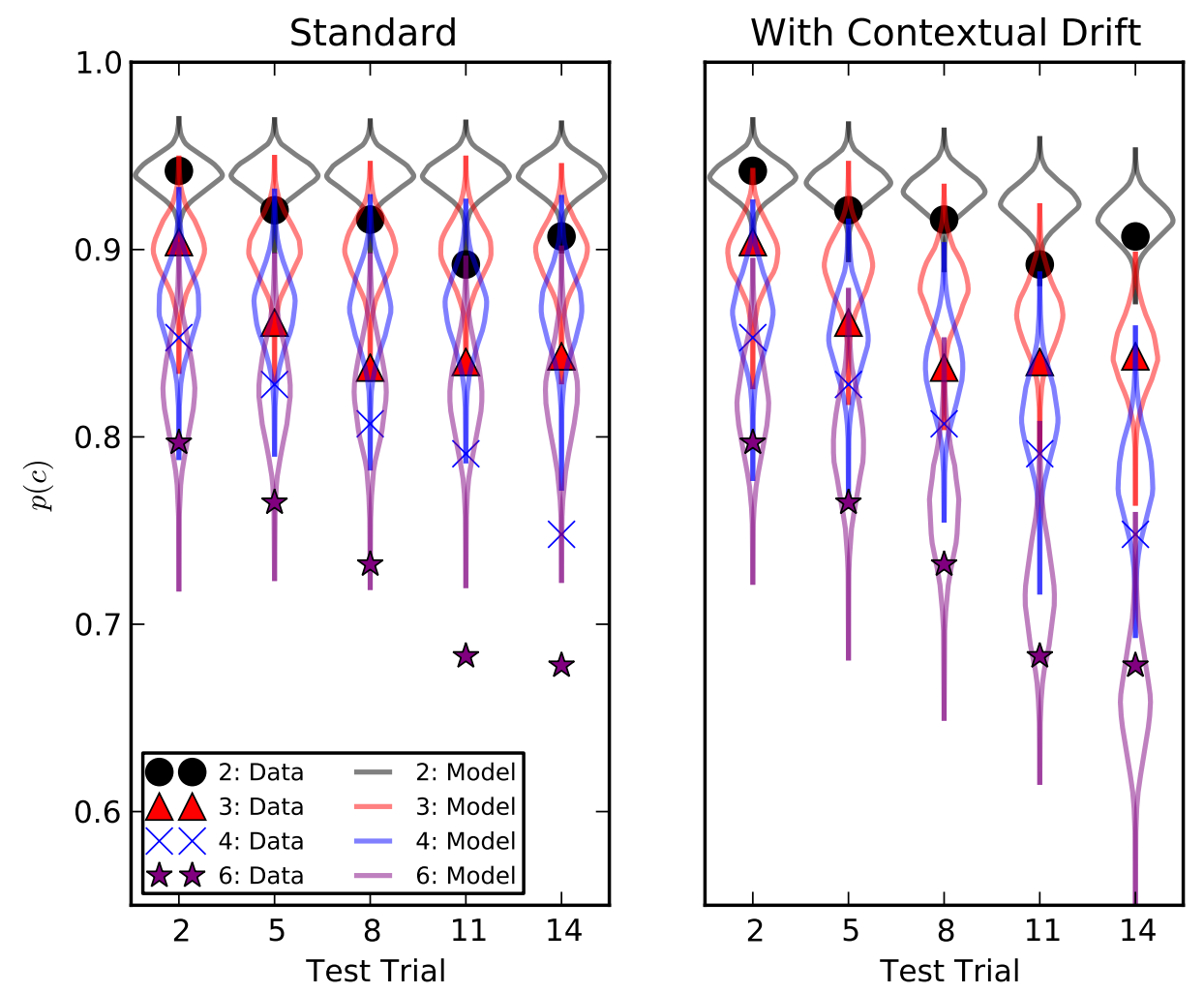

Figure 4.2: Group data and model predictions for the Murdock and Anderson (1975) paradigm, where the number of choices on a forced choice recognition test was manipulated between two and six. The left panel shows the model predictions without parameter modification, while the right panel shows the model's predictions each item on a test trial multiplies the mean match to context by .9985 . Note: The depicted data reflect averages across serial positions on the study list. 
test trials. In a control condition, in all category exemplars were randomized through the test block, performance decreased monotonically through testing. Malmberg et al. (2012) argued that these results are not only consistent with item noise theories, which predict that the magnitude of interference is dependent on the similarity of the cues to the contents of memory, but are also comparable to the release from proactive interference (PI) results of Wickens and colleagues (D. D. Wickens, 1970; D. D. Wickens, Born, \& Allen, 1963). In release from PI paradigms, recall of trigrams is found to decline over trials, which has been attributed to a buildup of PI from the preceding trials (Keppel \& Underwood, 1962). However, when the category of the to-be tested material is suddenly shifted, such as from digits to consonants, recall improves to around the level of the first trial. This effect has been dubbed release from PI because it is as if the shift in the similarity of the learned material prevents memory from suffering any interference from previous trials.

While the data of Malmberg et al. are compelling, their interpretation that the release from PI effect in their data is a consequence of item noise critically assumes that the original release from PI phenomenon observed by Wickens and colleagues was also due to the similarity of the item representations. An alternative conception is that release from PI is generally due to usage of new cues at retrieval when studied categories are switched (Gardiner, Craik, \& Birtwistle, 1972; Humphreys \& Tehan, 1992; Tehan \& Humphreys, 1995, 1996; Watkins \& Watkins, 1975). Watkins and Watkins (1975) proposed a cuing explanation of the release from PI phenomenon in their description of the cue overload principle, which states that performance degrades as the number of items associated with a cue is increased. By their view, when the category of the to-be tested material is suddenly switched, participants use a new category cue to guide their retrieval which exempts items from earlier trials to enter into the sampling set at recall.

A cuing explanation can similarly be given for the results of Malmberg et al., in that an obvious switch of the categories midway through testing may have prompted subjects to use a new category cue in conjunction with the item cue to overcome the contextual drift that had occurred throughout the test. Additionally, while there was a release from PI effect that was observed when large blocks of items were tested, no release from PI was 
observed when categories were shifted every five trials during testing, which is inconsistent with the item noise account of the testing decrement. One possibility is that there are costs associated with switching category cues that make it less likely when blocks are short, which is a view that is endorsed by the original authors: "It is less clear how long a block must be in order to observe a relase from output interference. There are likely costs associated with switching the contents of retrieval cues, say from emphasizing one set of item features reprsenting category membership rather than another set." (Malmberg et al., 2012, p. 4).

\subsubsection{Evidence for Differentiation Models of Recognition Memory}

Throughout this article, I have restricted discussion of item noise to simple global matching models that predict detrimental effects of list length and list strength on recognition memory performance when the item representations bear similarity to each other. Another class of models, referred to as differentiation models, were introduced to predict the null list strength effect in recognition memory. These models include a variant of the SAM model (Shiffrin et al., 1990) along with the REM (Shiffrin \& Steyvers, 1997) and SLiM (McClelland \& Chappell, 1998) models. In differentiation models, the first presentation of a study list item creates a new memory trace corresponding to that item, whereas subsequent reptitions update that memory trace. The repetitions not only make the representation more responsive to its own cue, but also makes the representation less similar to other items. Thus, strength has the functional effect of decreasing the item noise among the stored item representations with increasing strength, whereas more traditional item noise models predict increasing item noise with increased strength. Increases in list length do not induce differ-

entiation but instead create new memory traces, and thus differentiation models predict a detrimental effect of list length on recognition memory performance.

The original motivation behind the differentiation mechanism was to predict a dissociation between list length and list strength effects. As previously mentioned, while there were many published effects showing detrimental effects of increasing list length, Dennis and colleagues (e.g.: Dennis et al., 2008) have demonstrated that these appear to be due to confounds present in list length designs that show no effect of list length for word stimuli when 
controlled. While non-linguistic stimuli such as fractals and faces show worse performance in longer lists (Kinnell \& Dennis, 2012), the same stimuli appear to be susceptible to list strength effects as well (Osth et al., 2014). With the exception of face images, which appear to be more susceptible to manipulations of list length than list strength, in the data and modeling from my fits there appeared to be no dissociation between the effects of list length and list strength, as stimuli appeared to be affected negatively by both manipulations or they were not affected. Thus, there is not strong evidence for a dissociation between list length and list strength effects that warrants a differentiation mechanism.

Another prediction of differentiation models is the strength based mirror effect (Criss, 2006). Because increased strength makes a stored memory trace less confusable with other item representations, lures will exhibit considerably less similarity to the contents of memory when the memory traces are strong than when they are weak, making it such that the false alarm rate should reduce as memory traces are strengthened. As stated previously, this pattern is robustly observed in item recognition (Hirshman, 1995; Stretch \& Wixted, 1998b) and associative recognition (Clark \& Shiffrin, 1992; Hockley \& Niewiadomski, 2007; Osth \& Dennis, 2013). An alternative explanation of the strength based mirror effect is that it is caused by a criterion shift. That is, higher expected memory strength for a tested study list may cause the participant to adopt a stricter response criterion for the tested items, reducing the false alarm rate.

To counter the criterion shift argument, Criss $(2009,2010)$ has presented evidence in support of the predictions of differentiation models by demonstrating that memory strength for lures is reduced under conditions of higher strength. Criss (2009) tested participants under pure weak and pure strong conditions and asked them to indicate their confidence on a 20 point scale. Distributions of subjective memory strength were lower for lures following a pure strong list than following a pure weak list. To counter the argument that this was the product of a criterion shift, a manipulation of target probabilities on the test list did not have any effect on the strength estimates for lures, despite the fact that it affected bias on their yes/no repsonses. In addition, Criss (2010) estimated parameters of the Ratcliff diffusion model (Ratcliff, 1978; Ratcliff \& McKoon, 2008) and found that drift rates for 
lures were lower in a pure strong condition than in a pure weak condition, whereas a target probability manipulation only affected the starting point of evidence accumulation and exhibited no effect on the drift rates.

A difficulty in interpretting these results is that they do not only support differentiation models, but additionally support the overall class of likelihood ratio models. This model, which employs a likelihood ratio transformation of the memory strengths, not only predicts lower FAR under conditions of higher strength but also predicts that the log likelihood ratio distribution for lures should have also exhibit a lower mean than the corresponding distribution for lures studied in a weak study list.

A critical difference between the model I presented and the differentiation approach lies in what generates a shift in the distribution of evidence for lures when strength is increased. In differentiation models, the strength based mirror effect is produced by encoding processes that produce more resilient memory traces. In the model, it is the higher expected memory strength during a test that holds lures to a higher standard of evidence, producing lower likelihood ratios. Starns et al. (2010) noted that when a list of items is strengthened, both the encoding conditions and test expectations are confounded with each other. To separate the two accounts, they had participants study mixed lists of strong and weak items, but manipulated test expectations by only testing participants on the weak items or the strong items while informing participants about the strength composition of the test list. Differentiation models predict lower false alarm rates for stronger study lists regardless of what is expected on the test list. In contrast, false alarm rates were predicted by test expectations, as lower false alarm rates were observed in the strong test lists and higher false alarm rates were observed in the weak test lists.

A potential counter-argument to the results is that both differentiation and test expectations play a role in producing the strength based mirror effect. However, Starns et al. (2010) manipulated strength at two different levels on their mixed strength lists. On some lists, the strong items were presented twice (strong $2 \mathrm{X}$ condition), whereas on others, the strong items were presented five times (strong $5 \mathrm{X}$ condition). False alarm rates in the pure weak tests were identical in both the strong $2 \mathrm{X}$ and strong $5 \mathrm{X}$ conditions, which can be easily 
explained by assuming that in both lists, the lure items were held to the same expectations of memory strength. A differentiation model that also uses expected memory strength to alter the likelihood ratios predicts a higher FAR in the strong $5 \mathrm{X}$ condition because those memory traces are more differentiated than in the strong $2 \mathrm{X}$ condition. Subsequent investigations have also found that distributions of subjective memory strength (Starns, White, \& Ratcliff, 2012) and drift rates for lures (Starns, Ratcliff, \& White, 2012) are shifted when test expectations are manipulated following a mixed strength study list.

Thus, the available evidence suggests that differentiation is not necessary to explain either the null list strength effect or the strength based mirror effect. However, I do not mean to suggest that there is no mechanism of differentiation. Differentiation of item representations over the long term is both plausible and useful in describing how item representations evolve with experience even in cognitive domains outside of memory. For instance, McClelland et al. (1995) conducted simulations of the Rumelhart (1990) network of concept learning and demonstrated how differentiation occurs over training. The network is trained on propositions such as "A robin is a bird" and "A tree has branches." McClelland et al. (1995) discovered that during the initial training, all of the agents (robin, tree, etc.) exhibited similar hidden layer representations regardless of how similar they were to each other. As training proceeded to its conclusion, the hidden layer representations diverged and dissimilar entitites (such as robin and tree) exhibited dissimilar hidden layer representations.

One distinction between the differentiation of concepts observed by McClelland et al. and the episodic differentiation models such as SAM, REM, and SLiM, however, is that the episodic models are multiple trace models where separate copies of the item representations are stored in memory. Additionally, the episodic models posit that when a stimulus is presented in a new context, a new episodic trace is created and it is during subsequent presentations within that new context that differentiation of the episodic trace occurs (Criss, 2006, 2009). In the Rumelhart (1990) network, there is no obvious distinction between creating and differentiating representations, and differentiation operates on a larger timescale than that of a study list in a recognition memory experiments. Below, I posit that long-term differentiation may be able to explain the differences between the item noise estimates of 
linguistic and non-linguistic stimuli.

\subsection{Differences Between Linguistic and Non-Linguistic Stim- uli}

Another finding in the parameter estimates of the model was the higher estimates of item mismatch variability and item noise for fractals and faces than for words and scenes. Why would representations of faces and scenes be more susceptible to item noise? One possibility that was initially raised by Kinnell and Dennis (2012) is that fractals and faces have more overlap in their representations, making them more likely to suffer from effects of list length and list strength.

But why would stimuli such as faces and fractals exhibit more overlap in their representations as opposed to words and scenes? One hypothesis is that long term experience unitizes stimulus representations to minimize within-class similarity. That is, untrained stimuli begin with overlapping representations, making them susceptible to other stored stimuli that they are similar to and thus they suffer from high degrees of item noise and background noise. However, as stimuli become unitized through training, they exhibit less similarity to other stored stimuli but still match their own previously stored representations, making them susceptible to context noise.

This hypothesis was initially proposed by Reder, Angstadt, Cary, Erickson, and Ayers (2002) to explain the non-monotonic relationship between word frequency and recognition memory performance. While low frequency words outperform high frequency words, very low frequency words exhibit worse performance than low frequency words and exhibit higher hit rates and false alarm rates (Wixted, 1992; Chalmers, Humphreys, \& Dennis, 1997; Zechmeister, Curt, \& Sebastian, 1978). Reder et al. (2002) conducted a training study with pseudowords and found that initial training increased hit rates and false alarm rates, but after six weeks of training, a mirror effect was evident with respect to training, with less frequently trained pseudowords exhibiting higher hit rates and lower false alarm rates. Similar results were found by Nelson and Shiffrin (2013) using Chinese characters 
as the stimuli. A training study using very low frequency words conducted by Chalmers and Humphreys (1998) found that definitions might facilitate unitization, in that training without definitions hurt performance on the words, whereas training with definitions improved performance on the very low frequency words to around the level of low frequency words. Converging evidence for the unitization hypothesis could be found by observing how susceptible stimuli are to list length effects through training.

Item noise estimates for natural scene photographs closely resembled those of single words. Aside from the generally higher performance for pictorial stimuli (Brady, Konkle, Alvarez, \& Oliva, 2008; Shepard, 1967; Standing, 1973), representations of scenes may resemble words due to the fact that labels can easily be applied to segments of the images. The idea that pictorial stimuli have linguistic representations was posited by Paivio (1971, 1976), who argued that pictures have "dual codes" possessing both perceptual and linguistic information. Evidence for this hypothesis comes from the finding that recognition performance for pictures is still superior to single words when the test stimuli are labels instead of the pictures themselves (Paivio, 1976; Madigan, 1983). Similarly, a mirror effect that resembles the word frequency mirror effect can be found for pictorial stimuli. Karlsen and Snodgrass (2004) found that both pictures and words rated high in familiarity exhibited lower hit rates and higher false alarm rates than those rated low in familiarity, whereas in free recall both pictures and words rated high in familiarity were better recalled.

What kind of model could explain the unitization process? Decreasing the within-class similarity for a stimulus set is very similar to the principle of differentiation employed in differentiation models such as SAM, REM, and SLiM. However, a critical distinction I would like to address is that differentiation models of episodic memory operate over the short term, whereas I argue that a differentiation-like process operates over longer time scales. Specifically, in short-term differentiation models, presentation of a familiar stimulus in a new context creates a new representation and subsequent presentations refine that newly created representation (Criss, 2006, 2009). I hypothesize that there is no distinction between creating and updating episodic item representations. Familiarization with a stimulus decreases its overlap with other stimuli of the same class, minimizing item noise and back- 
ground noise, but increasing the susceptibility of the stimulus to context noise as it becomes bound to more contexts with further experience.

The current modeling exercise gives no insight as to how the minimization of the overlap might occur. A complete model of episodic and general memory would need to describe how the item representations evolve with experience and would require long-term training data to constrain the parameters that guide the transitions (see Nelson \& Shiffrin, 2013, for one such attempt). One possibe mechanism for stimulus unitization is competitive autoassociation. While the current model only describes associations between the item and context layers, within-layer connections for the items could be implemented as well (e.g.: J. A. Anderson, Silverstein, Ritz, \& Jones, 1977). Distributed item representations could become more sparse over long term experience if each item layer is competitive such that only a small number of units can be active at a given time, which is achieved in neural networks by usage of the k-winner takes-all (kWTA) algorithm. Norman and O'Reilly (2003) used the k-WTA algorithm in recognition memory to show that item representations could become more sparse as a consequence of experience, indicating that such an approach shows promise for this endeavor.

\subsection{Other Sources of Variability in Recognition Memory}

One source of variability that was not included in the model fit was trial-to-trial variability in criterion placement. A number of criterion noise models of signal detection have been developed that include such variability (Benjamin, Diaz, \& Wee, 2009; Mueller \& Weidemann, 2008; Wickelgren, 1968). However, one of the critical challenges in incorporating criterion variability into a model fit is identifying its contribution from the variability in memory strength. Benjamin et al. (2009) proposed that such measurement can be done using an ensemble recognition paradigm in which participants give yes/no decisions not to one stim-

ulus, but to ensembles of old and new words that vary in the number of words contained in the ensemble, with the logic being that ensemble size constrains memory strength variability but does not affect criterion variability. Given that none of the experiments contain 
manipulations of ensemble size, I do not have the ability to constrain trial-to-trial criterion variability. Additionally, the approach of Benjamin et al. (2009) remains somewhat controversial, as Kellen, Klauer, and Singmann (2012) have argued that Benjamin et al. (2009) severely over-estimated criterion noise in their dataset. They demonstrated in their model fit that if decision criteria are allowed to shift across the ensemble sizes, criterion variability estimates decrease dramatically.

Another approach that avoids the usage of new paradigms involves usage of response time models to quantify decision noise. The majority of current sequential sampling models of response time employ trial-to-trial variability in the starting point of evidence accumulation (S. D. Brown \& Heathcote, 2008; Ratcliff et al., 1999; Usher \& McClelland, 2001), a source of decision noise which has been described as analogous to criterion variability in a signal detection framework (Benjamin, 2013; Benjamin et al., 2009). While sequential sampling models are quite successful in their ability to measure aspects of the decision-making process such as response caution, bias, and the strength of the evidence, they are not able to specify the contributions of encoding and interference that are contributing to the evidence used in the decision. One possible extension of the model is to use a back-end sequential sampling model to produce decisions, allowing the model to not only make response time predictions but also to estimate the contribution of decision noise. I was not able to undertake such an approach in the current investigation because much larger numbers of correct and error responses are needed in each response category than are contained in the present datasets to properly estimate the response time distributions (Ratcliff \& McKoon, 2008).

\subsection{Conclusion}

My fits of a global matching model which parameterizes the matches and mismatches to item and context demonstrated that the bulk of interference comes from experiences prior to the list-learning episode (context noise and background noise), with confusable stimuli such as fractals and faces exhibiting at most small contributions of item noise. While these parameter estimates may seem counter-intuitive, they appear to be quite consistent with a 
wide variety of findings in the recognition memory literature as well as theories in cognitive neuroscience that advocate sparse distributed item representations.

Additionally, the model was able to fit quite well to a variety of manipulations of stimulus class, strength, list length, list strength, study-test delay, and word frequency. Several of these variables have been considered challenging to the first generation of global matching models, while the results of my modeling work suggest that the initial global matching models are quite capable of addressing these results by parameterizing the similarities between the representations. An additional advantage to parameterizing similarities instead of vectors is that it obviates the need for a vector size parameter, which affects the identifiability of the model parameters (Montenegro et al., 2011; Myung et al., 2007). Small effects of list length and list strength are well accommodated by low values of item mismatch, unequal variance between targets and lures can be explained with item and context match variability, and mirror effects are capably explained by a likelihood ratio transformation of memory strengths. The global matching model I present is both simple and tractable, and shows promise in being extended to other memory tasks. 


\section{REFERENCES}

Aminoff, E. M., Clewett, D., Freeman, S., Frithsen, A., Tipper, C., Johnson, A., et al. (2012). Individual differences in shifting decision criterion: A recognition memory study. Memory $\&$ Cognition, 40(7), 1016-1030.

Anderson, J. A., Silverstein, J. W., Ritz, S. A., \& Jones, R. S. (1977). Distinctive Features, Categorical Perception, and Probability Learning: Some Applications of a Neural Model. Psychological Review, 84(5), 413-451.

Anderson, J. R., \& Bower, G. H. (1972). Recognition and retrieval processes in free recall. Psychological Review, 79(2), 97-123.

Annis, J., Malmberg, K. J., Criss, A. H., \& Shiffrin, R. M. (2013). Sources of interference in recognition testing. Journal of Experimental Psychology: Learning, Memory, and Cognition, 39(5), 1365-1376.

Arndt, J., \& Hirshman, E. (1998). True and False Recognition in MINERVA2: Explanations from a Global Matching Perspective. Journal of Memory and Language, 39, 371-391.

Benjamin, A. S. (2013). Where is the criterion noise in recognition? (Almost) everyplace you look: Comment on Kellen, Klauer, and Singmann (2012). Psychological Review, $120(3), 720-726$.

Benjamin, A. S., Diaz, M., \& Wee, S. (2009). Signal detection with criterion noise: Applications to recognition memory. Psychological Review, 116(1), 84-115.

Botvinick, M. M., \& Plaut, D. C. (2006). Short-term memory for serial order: A recurrent neural netowrk model. Psychological Review, 113, 201-233.

Bowles, N. L., \& Glanzer, M. (1983). An analysis of interference in recognition memory. 
Memory and Cognition, 11, 307-315.

Brady, T. F., Konkle, T., Alvarez, G. A., \& Oliva, A. (2008). Visual long-term memory has a massive storage capacity for object details. Proceedings of the National Academy of Sciences, $105(38), 14325-14329$.

Brown, G. D. A., Neath, I., \& Chater, N. (2007). A temporal ratio model of memory. Psychological Review, 114, 539-576.

Brown, G. D. A., Preece, T., \& Hulme, C. (2000). Oscillator-based memory for serial order. Psychological Review, 107, 127-181.

Brown, J., Lewis, V. J., \& Monk, A. F. (1977). Memorability, word frequency and negative recognition. The Quarterly Journal of Experimental Psychology, 29(3), 461-473.

Brown, S. D., \& Heathcote, A. (2008). The simplest complete model of choice response time: Linear ballistic accumulation. Cognitive Psychology, 57, 153-178.

Buchler, N. G., Faunce, P., Light, L. L., Gottfredson, N., \& Reder, L. M. (2011). Effects of repetition on associative recognition in young and older adults: Item and associative strengthening. Psychology and Aging, 26(1), 111-126.

Buchler, N. G., Light, L. L., \& Reder, L. M. (2008). Memory for items and associations: Distinct representations and processes in associative recognition. Journal of Memory and Language, 59, 183-199.

Cary, M., \& Reder, L. M. (2003). A dual-process account of the list-length and strengthbased mirror effects in recognition. Journal of Memory and Language, 49(2), 231-248.

Chalmers, K. A., \& Humphreys, M. S. (1998). Role of generalized and episode specific memories in the word frequency effect in recognition. Journal of Experimental PsychologyLearning Memory and Cognition, $24(3), 610-632$.

Chalmers, K. A., Humphreys, M. S., \& Dennis, S. (1997). A naturalistic study of the word frequency effect in episodic recognition. Memory $\&$ Cognition, 25(6), 780-784.

Chappell, M., \& Humphreys, M. S. (1994). An auto-associative neural network for sparse representations: Analysis and application to models of recognition and cued recall. Psychological Review, 101, 103-128.

Cho, K. W., \& Neely, J. H. (2013). Null category-length and target-lure relatedness effects 
in episodic recognition: A constraint on item-noise interference models. The Quarterly Journal of Experimental Psychology, 66(7), 1331-1355.

Clark, S. E. (1997). A familiarity-based account of confidence-accuracy inversions in recognition memory. Journal of Experimental Psychology: Learning, Memory, and Cognition, 23(1), 232-238.

Clark, S. E., \& Gronlund, S. D. (1996). Global matching models of recognition memory: How the models match the data. Psychonomic Bulletin and Review, 3(1), 37-60.

Clark, S. E., \& Hori, A. (1995). List length and overlap effects in forced-choice associative recognition. Memory \& Cognition, 23(4), 456-461.

Clark, S. E., \& Shiffrin, R. M. (1992). Cuing Effects and Associative Information in Recognition Memory. Memory \& Cognition, 20(5), 580-598.

Cleary, A. M., Curran, T., \& Greene, R. L. (2001). Memory for detail in item versus associative recognition. Memory \&6 Cognition, 29(3), 413-423.

Criss, A. H. (2006). The consequences of differentiation in episodic memory: Similarity and the strength based mirror effect. Journal of Memory and Language, 55, 461-478.

Criss, A. H. (2009). The distribution of subjective memory strength: List strength and response bias. Cognitive Psychology, 59, 297-319.

Criss, A. H. (2010). Differentiation and response bias in episodic memory: Evidence from reaction time distributions. Journal of Experimental Psychology: Learning, Memory, 83 Cognition, 36(2), 484-499.

Criss, A. H., Malmberg, K. J., \& Shiffrin, R. M. (2011). Output interference in recognition memory. Journal of Memory and Language, 64, 316-326.

Criss, A. H., \& Shiffrin, R. M. (2004). Context-noise and item-noise jointly determine recognition memory: A comment on Dennis and Humphreys (2001). Psychological Review, 111, 800-807.

Dalezman, J. J. (1976). Effects of output order on immediate, delayed, and final recall performance. Journal of Experimental Psychology: Human Learning and Memory, 2(5), 597-608.

Davelaar, E. J. (2007). Sequential retreival and inhibition of parallel (re)activated repre- 
sentations: A neurocomputational comparison of competitive queuing and resampling models. Adaptive Behavior, 15(1), 51-71.

Davelaar, E. J., Goshen-Gottstein, Y., Ashkenazi, A., Haarmann, H., \& Usher, M. (2005). The demise of short-term memory revisited: empirical and computational investigations of recency effects. Psychological Review, 112(1), 3-42.

DeCarlo, L. T. (2002). Signal detection theory with finite mixture distributions: Theoretical developments with applications to recognition memory. Psychological Review, 109 (4), $710-721$.

DeCarlo, L. T. (2007). The mirror effect and mixture signal detection theory. Journal of Experimental Psychology: Learning, Memory, and Cognition, 33(1), 18-33.

Deese, J. (1959). On the prediction of the occurrence of particular verbal intrusions in immediate recall. Journal of Experimental Psychology, 58, 17-22.

Dennis, S., \& Chapman, A. (2010). The inverse list length effect: A challenge for pure exemplar models of recognition memory. Journal of Memory and Language, 63, 416424.

Dennis, S., \& Humphreys, M. S. (2001). A context noise model of episodic word recognition. Psychological Review, 108(2), 452-478.

Dennis, S., Lee, M. D., \& Kinnell, A. (2008). Bayesian Analysis of Recognition Memory: The Case of the List-Length Effect. Journal of Memory and Language, 59, 361-376.

Dewhurst, S. A., \& Anderson, S. J. (1999). Effects of exact and category repetition in true and false recognition memory. Memory $\&$ Cognition, 27, 664-673.

Dong, T. (1972). Cued partial recall of categorized words. Journal of Experimental Psychology, 93(1), 123-129.

Dosher, B. A., \& Rosedale, G. (1989). Integrated retrieval cues as a mechanism for priming in retrieval from memory. Journal of Experimental Psychology: General, 118(2), $191-211$.

Egan, J. P. (1958). Signal detection theory and ROC analysis (Tech. Rep.). Hearing and Communication Laboratory.

Estes, W. K., \& Maddox, W. T. (2005). Risks of drawing inferences about cognitive 
processes from model fits to individual versus average performance. Psychonomic Bulletin \& Review, 12(3), 403-408.

Farrell, S. (2012). Temporal clustering and sequencing in short-term memory and episodic memory. Psychological Review, 119(2), 223-271.

Gallo, D. A., Sullivan, A. L., Daffner, K. R., Schacter, D. L., \& Budson, A. E. (2004). Associative recognition in Alzheimer's disease: Evidence for impaired recall-to-reject. Neuropsychology, 18(3), 556-563.

Gardiner, J. M., Craik, F. I. M., \& Birtwistle, J. (1972). Retrieval cues and release from proactive inhibition. Journal of Verbal Learning and Verbal Behavior, 11, 778-783.

Gillund, G., \& Shiffrin, R. M. (1984). A retrieval model for both recognition and recall. Psychological Review, 91(1), 1-67.

Glanzer, M., \& Adams, J. K. (1985). The mirror effect in recognition memory. Memory and Cognition, 13(1), 8-20.

Glanzer, M., \& Adams, J. K. (1990). The Mirror Effect in Recognition Memory: Data and Theory. Journal of Experimental Psychology: Learning, Memory, and Cognition, $16(1), 5-16$.

Glanzer, M., Adams, J. K., \& Iverson, G. (1991). Forgetting and the mirror effect in recognition memory: Concentering of underlying distributions. Journal of Experimental Psychology: Learning, Memory, and Cognition, 17(1), 81-93.

Glanzer, M., Adams, J. K., Iverson, G. J., \& Kim, K. (1993). The regularities of recognition memory. Psychological Review, 100(3), 546-567.

Glanzer, M., \& Bowles, N. (1976). Analysis of the word-frequency effect in recognition memory. Journal of Experimental Psychology: Human leanring and memory, 2(1), $21-31$.

Glanzer, M., Hilford, A., \& Maloney, L. T. (2009). Likelihood ratio decisions in memory: Three implied regularities. Psychonomic Bulletin \& Review, 16(3), 431-455.

Glanzer, M., Kim, K., Hilford, A., \& Adams, J. K. (1999). Slope of the receiver-operating characteristic in recognition memory. Journal of Experimental Psychology: Learning, Memory, and Cognition, 25(2), 500-513. 
Gronlund, S. D., \& Elam, L. E. (1994). List-length effect: Recognition accuracy and variance of underlying distributions. Journal of Experimental Psychology: Learning, Memory and Cognition, 20, 1335-1369.

Heathcote, A. (2003). Item recognition memory and the receiver operating characteristic. Journal of Experimental Psychology: Learning, Memory, and Cognition, 29(6), 12101230.

Henson, R. N. A. (1998). Short-term memory for serial order: The start-end model. Cognitive Psychology, 36, 73-137.

Hilford, A., Glanzer, M., \& Kim, K. (1997). Encoding, repetition, and the mirror effect in recognition memory: Symmetry in motion. Memory \& Cognition, 25(5), 593-605.

Hintzman, D. L. (1988). Judgments of Frequency and Recognition Memory in a MultipleTrace Memory Model. Psychological Review, 95(4), 528-551.

Hirshman, E. (1995). Decision processes in recognition memory: Criterion shifts and the list-strength paradigm. Journal of Experimental Psychology: Learning, Memory, and Cognition, 21(2), 302-313.

Hockley, W. E., \& Cristi, C. (1996a). Tests of encoding tradeoffs between item and associative information. Memory \& Cognition, 24(2), 202-216.

Hockley, W. E., \& Cristi, C. (1996b). Tests of the separate retrieval of item and associative information using a frequency-judgment task. Memory $\mathscr{E}$ Cognition, 24 (6), 796-811.

Hockley, W. E., \& Niewiadomski, M. W. (2007). Strength-based mirror effects in item and associative recognition: Evidence for within-list criterion changes. Memory $\&$ Cognition, 35(4), 679-688.

Howard, M. W., \& Kahana, M. J. (2002). A distributed representation of temporal context. Journal of Mathematical Psychology, 46, 268-299.

Humphreys, M. S., Bain, J. D., \& Pike, R. (1989). Different Ways to Cue a Coherent Memory System - a Theory for Episodic, Semantic, and Procedural Tasks. Psychological Review, 96(2), 208-233.

Humphreys, M. S., Pike, R., Bain, J. D., \& Tehan, G. (1989). Global matching: A comparison of the SAM, Minerva II, Matrix and TODAM models. Journal of Mathematical 
Psychology, 33, 36-67.

Humphreys, M. S., \& Tehan, G. (1992). A simultaneous examination of recency and cuing effects. In A. F. Healy, S. M. Kosslyn, \& R. M. Shiffrin (Eds.), From learning theory to cognitive processes: Essays in honor of William K. Estes (Vol. 2, pp. 143-159). Erlbaum.

Jang, Y., \& Huber, D. E. (2008). Context retrieval and context change in free recall: Recalling from long-term memory drives list isolation. Journal of Experimental Psychology: Learning, Memory, and Cognition, 34(1), 112-127.

Jang, Y., Mickes, L., \& Wixted, J. T. (2012). Three tests and three corrections: A comment on Koen and Yonelinas (2010). Journal of Experimental Psychology: Learning, Memory, and Cognition, 38(2), 513-523.

Johns, B. T., Jones, M. N., \& Mewhort, D. J. K. (2012). A synchronization account of false recognition. Cognitive Psychology, 65(4), 486-518.

Jordan, M. I. (2004). Graphical Models. Statistical Science, 19, 140-155.

Kahana, M. J., Rizzuto, D. S., \& Schneider, A. R. (2005). Theoretical correlations and measured correlations: Relating recognition and recall in four distributed memory models. Journal of Experimental Psychology: Learning Memory and Cognition, 31(5), 933-953.

Kantner, J., \& Lindsay, D. S. (2012). Response bias in recognition memory as a cognitive trait. Memory \& Cognition, 40, 1163-1177.

Karlsen, P. J., \& Snodgrass, J. G. (2004). The word-frequency paradox for recall/recognition occurs for pictures. Psychological Research, 68, 271-276.

Kellen, D., Klauer, K. C., \& Singmann, H. (2012). On the measurement of criterion noise in signal detection theory: The case of recognition memory. Psychological Review, $119(3), 457-479$.

Kelley, R., \& Wixted, J. T. (2001). On the Nature of Associative Information in Recognition Memory. Journal of Experimental Psychology: Learning, Memory, and Cognition, $27(3), 701-722$.

Keppel, G., \& Underwood, B. (1962). Proactive inhibition in short-term retention of single 
items. Journal of Verbal Learning and Verbal Behavior, 1, 153-161.

Kim, K., \& Glanzer, M. (1993). Speed versus accuracy instructions, study time, and the mirror effect. Journal of Experimental Psychology: Learning, Memory, and Cognition, $19(3), 638-652$.

Kim, K., \& Glanzer, M. (1995). Intralist interference in recognition memory. Journal of Experimental Psychology: Learning, Memory, and Cognition, 21(5), 1096-1107.

Kinnell, A., \& Dennis, S. (2011). The list length effect in recognition memory: An analysis of potential confounds. Memory \& Cognition, 39, 348-363.

Kinnell, A., \& Dennis, S. (2012). The role of stimulus type in list length effects in recognition memory. Memory \& Cognition, 40, 311-325.

Klein, K. A., Shiffrin, R. M., \& Criss, A. H. (2007). Putting context in context. In The Foundations of Remembering: Essays in Honor of Henry L. Roediger III (pp. 171-189). Psychology Press.

Koen, J. D., \& Yonelinas, A. P. (2010). Memory variability is due to the contribution of recolleciton and familiarity, not to encoding variability. Journal of Experimental Psychology: Learning, Memory, and Cognition, 36(6), 1536-1542.

Koen, J. D., \& Yonelinas, A. P. (2013). Still no evidence for the encoding variability hypothesis: A reply to Jang, Mickes, and Wixted (2012) and Starns, Rotello, and Ratcliff (2012). Journal of Experimental Psychology: Learning, Memory, and Cognition, 39(1), 304-312.

Konkle, T., Brady, T. F., Alvarez, G., \& Oliva, A. (2010). Conceptual distinctiveness supports detailed visual long-term memory for real-world objects. Journal of Experimental Psychology: General, 139(3), 558-578.

Kumaran, D., \& McClelland, J. L. (2012). Generalization through the recurrent interaction of episodic memories: A model of the hippocampal system. Psychological Review, $119(3), 573-616$.

Landauer, T. K., \& Dumais, S. T. (1997). A solution to Plato's problem: The Latent Semantic Analysis theory of the acquisition, induction, and representation of knowledge. Psychological Review, 104, 211-240. 
Lee, M. D. (2008). Three case studies in the Bayesian analysis of cognitive models. Psychonomic Bulletin \& Review, 15(1), 1-15.

Lee, M. D. (2011). How cognitive modeling can benefit from hierarchical Bayesian models. Journal of Mathematical Psychology, 55, 1-7.

Lee, M. D., \& Vanpaemel, W. (2008). Exemplars, prototypes, similarities, and rules in category representation: An example of Hierarchical Bayesian Analysis. Cognitive Science, 32(8), 1403-1424.

Lehman, M., \& Malmberg, K. J. (2013). A buffer model of memory encoding and temporal correlations in retrieval. Psychological Review, 120(1), 155-189.

Lewandowsky, S., \& Murdock, B. B. (1989). Memory for serial order. Psychological Review, $96,25-57$.

Light, L. L., Patterson, M. M., Chung, C., \& Healy, M. R. (2004). Effects of repetition and response deadline on associative recognition in young and older adults. Memory E Cognition, 32(7), 1182-1193.

Madigan, S. (1983). Picture memory. In J. C. Yuillie (Ed.), Imagery, memory, and cognition: Essays in honor of Allan Paivio (pp. 65-89). Erlbaum.

Maguire, A. M., Humphreys, M. S., Dennis, S., \& Lee, M. D. (2010). Global similarity accounts of embedded-category designs: Tests of the global matching models. Journal of Memory and Language, 63(2), 131-148.

Malmberg, K. J., Criss, A. H., Gangwani, T. H., \& Shiffrin, R. M. (2012). Overcoming the negative consequences of interference from recognition memory testing. Psychological Science, 23(2), 115-119.

Malmberg, K. J., \& Shiffrin, R. M. (2005). The "one-shot" hypothesis for context storage. Journal of Experimental Psychology: Learning, Memory, and Cognition, 31(2), 322336.

Malmberg, K. J., \& Xu, J. (2007). On the flexibility and the fallibility of associative memory. Memory $\& 3$ Cognition, 35(3), 545-556.

Marr, D. (1971). A theory for archicortex. Philosophical Transactions of the Royal Society of London. Series B, Biological Sciences, 262(841), 23-81. 
McClelland, J. L., \& Chappell, M. (1998). Familiarity breeds differentiation: A subjectivelikelihood approach to the effects of experience in recognition memory. Psychological Review, 105(4), 724-760.

McClelland, J. L., McNaughton, B. L., \& O'Reilly, R. C. (1995). Why there are complementary learning systems in the hippocampus and neocortex: Insights from the successes and failures of connectionist models of learning and memory. Psychological Review, 102(3), 419-457.

Mensink, G. J., \& Raaijmakers, J. G. W. (1988). A model for interference and forgetting. Psychological Review, 95(4), 434-455.

Mickes, L., Johnson, E. M., \& Wixted, J. T. (2010). Continuous recollection versus unitized familiarity in associative recognition. Journal of Experimental Psychology: Learning, Memory, and Cognition, 36(4), 843-863.

Montenegro, M., Myung, J. I., \& Pitt, M. A. (2011). REM integral expressions. (Unpublished manuscript)

Morey, R. D., Pratte, M. S., \& Rouder, J. N. (2008). Problematic effects of aggregation in zROC analysis and a hierarchical modeling solution. Journal of Mathematical Psychology, 52, 376-388.

Mueller, S. T., \& Weidemann, C. T. (2008). Decision noise: An explanation for observed violations of signal detection theory. Psychonomic Bulletin \& Review, 15(3), 465494.

Murdock, B. B. (1962). The serial position effect of free recall. Journal of Experimental Psychology, 64(5), 482-488.

Murdock, B. B. (1982). A theory for the storage and retrieval of item and associative information. Psychological Review, 89(6), 609-626.

Murdock, B. B. (1985). Convolution and matrix systems: A reply to Pike. Psychological Review, 92(1), 130-132.

Murdock, B. B. (1997). Context and mediators in a theory of distributed associative memory (TODAM2). Psychological Review, 104(4), 839-862.

Murdock, B. B., \& Anderson, R. E. (1975). Encoding, storage, and retrieval of item informa- 
tion. In R. L. Solso (Ed.), Theories in cognitive psychology: The Loyola Symposium. Erlbaum.

Murdock, B. B., \& Kahana, M. J. (1993a). Analysis of the list-strength effect. Journal of Experimental Psychology: Learning, Memory, and Cognition, 19(3), 689-697.

Murdock, B. B., \& Kahana, M. J. (1993b). List-Strength and list-length effects: Reply to Shiffrin, Ratcliff, Murnane, and Nobel (1993). Journal of Experimental Psychology: Learning, Memory, and Cognition, 19(6), 1450-1453.

Murdock, B. B., \& Okada, R. (1970). Interresponse times in single-trial free recall. Journal of Experimental Psychology, 86(2), 263-267.

Murnane, K., \& Shiffrin, R. M. (1991a). Interference and the representation of events in memory. Journal of Experimental Psychology: Learning, Memory, and Cognition, $17(5), 855-874$.

Murnane, K., \& Shiffrin, R. M. (1991b). Word repetitions in sentence recognition. Memory E Cognition, 19(2), 119-130.

Myung, J. I., Montenegro, M., \& Pitt, M. A. (2007). Analytic expressions for the BCDMEM model of recognition memory. Journal of Mathematical Psychology, 51, 198-204.

Naveh-Benjamin, M. (2000). Adult age differences in memory performance: Tests of an associative deficit hypothesis. Journal of Experimental Psychology: Learning, Memory, and Cognition, 2000(26), 1170-1187.

Neely, J. H., \& Tse, C.-S. (2009). Category length produces an inverted-U discriminability function in episodic recognition memory. The Quarterly Journal of Experimental Psychology, 62(6), 1141-1172.

Nelson, A. B., \& Shiffrin, R. M. (2013). The co-evolution of knowledge and event memory. Psychological Review, 120(2), 356-394.

Nobel, P. A., \& Shiffrin, R. M. (2001). Retrieval processes in recognition and cued recall. Journal of Experimental Psychology: Learning, Memory, and Cognition, 27(2), 384413.

Norman, K. A., \& O'Reilly, R. C. (2003). Modeling hippocampal and neocortical contributions to recognition memory: A complementary leanring systems approach. Psycho- 
logical Review, 110(4), 611-646.

Norman, K. A., Tepe, K., Nyhus, E., \& Curran, T. (2008). Event-related potential correlates of interference effects on recognition memory. Psychonomic Bulletin and Review, $15(1), 36-43$.

Nosofsky, R. M. (1986). Attention, similarity and the idenitification-categorization relationship. Journal of Experimental psychology: General, 115, 39-57.

Nosofsky, R. M. (1991). Tests of an exemplar model for relating perceptual classification and recognition memory. Journal of Experimental Psychology: Human Perception and Performance, 17(1), 3-27.

O’Reilly, R. C., \& McClelland, J. L. (1994). Hippocampal conjunctive encoding, storage, and recall: Avoiding a trade-off. Hippocampus, 4(6), 661-682.

O'Reilly, R. C., \& Rudy, J. W. (2001). Conjunctive representations in learning and memory: Principles of cortical and hippocampal function. Psychological Review, 108(2), 311345.

Osth, A. F., \& Dennis, S. (2013). Associative recognition and the list strength paradigm. Memory \& Cognition. Online.

Osth, A. F., Dennis, S., \& Kinnell, A. (2014). Stimulus type and the list strength paradigm. Quarterly Journal of Experimental Psychology. Online.

Paivio, A. (1971). Imagery and verbal processes. Holt, Rinehart, \& Winston.

Paivio, A. (1976). Imagery in recall and recognition. In J. Brown (Ed.), Recall and recognition (pp. 103-129). Wiley.

Peixotto, H. E. (1947). Proactive inhibtion in the recognition of nonsense syllables. Journal of Experimental Psychology, 37(1), 81-91.

Pike, R. (1984). Comparison of Convolution and matrix distributed memory systems for associative recall and recognition. Psychological Review, 91(3), 281-294.

Plummer, M. (2003). JAGS: A program for analysis of Bayesian graphical models using Gibbs sampling.

Polyn, S. M., Norman, K. A., \& Kahana, M. J. (2009). A context maintenance and retrieval model of organizational processes in free recall. Psychological Review, 116(1), 129- 
156.

Pooley, J. P., Lee, M. D., \& Shankle, W. R. (2011). Understanding memory impairment with memory models and hierarchical Bayesian analysis. Journal of Mathematical Psychology, 47-56.

Pratte, M. S., \& Rouder, J. N. (2011). Hierarchical single- and dual process models of recognition memory. Journal of Mathematical Psychology, 55(1), 36-46.

Pratte, M. S., Rouder, J. N., \& Morey, R. D. (2010). Separating mnemonic process from participant and item effects in the assessment of ROC asymmetries. Journal of Experimental Psychology: Learning, Memory, and Cognition, 36(1), 224-232.

Raaijmakers, J. G. W., \& Shiffrin, R. M. (1981). Search of Associative Memory. Psychological Review, 88(2), 93-134.

Ratcliff, R. (1978). A theory of memory retrieval. Psychological Review, 85, 59-108.

Ratcliff, R., Clark, S. E., \& Shiffrin, R. M. (1990). List-strength effect : I. Data and discussion. Journal of Experimental Psychology: Learning Memory and Cognition, $16(2), 163-178$.

Ratcliff, R., \& Hockley, W. E. (1980). Repeated negatives in item recognition: Nonmonotonic lag functions. In R. S. Nickerson (Ed.), Attention and Performance VIII. Erlbaum.

Ratcliff, R., \& McKoon, G. (2008). The diffusion decision model: Theory and data for two-choice decision tasks. Neural Computation, 20, 873-922.

Ratcliff, R., McKoon, G., \& Tindall, M. (1994). Empirical generality of data from recognition memory: Receiver-operating characteristic functions and implications for the global memory models. Journal of Experimental Psychology: Learning, Memory, and Cognition, 20(4), 763-785.

Ratcliff, R., \& Murdock, B. B. (1976). Retrieval Processes in Recognition Memory. Psychological Review, 83(3), 190-214.

Ratcliff, R., Sheu, C. F., \& Gronlund, S. D. (1992). Testing global memory models using ROC curves. Psychological Review, 99(3), 518-535.

Ratcliff, R., \& Starns, J. J. (2009). Modeling confidence and response time in recognition 
memory. Psychological Review, 116(1), 59-83.

Ratcliff, R., Van Zandt, T., \& McKoon, G. (1999). Connectionist and diffusion models of reaction time. Psychological Review, 106(2), 261-300.

Reder, L. M., Angstadt, P., Cary, M., Erickson, M. A., \& Ayers, M. S. (2002). A reexamination of stimulus-frequency effects in recognition: Two mirrors for low- and high-frequency pseudowords. Journal of Experimental Psychology: Learning, Memory, and Cognition, 28(1), 138-152.

Roberts, W. A. (1972). Free recall of word lists varying in length and rate of presentation: A test of total-time hypotheses. Journal of Experimental Psychology, 92(3), 365-372.

Roediger, H. L. (1974). Inhibiting effects of recall. Memory $\&$ Cognition, 2(2), 261-269.

Roediger, H. L., \& McDermott, K. B. (1995). Creating false memories: Remembering words not present in lists. Journal of Experimental Psychology: Learning, Memory and Cognition, 21, 803-814.

Roediger, H. L., \& Schmidt, S. R. (1980). Output interference in the recall of categorized and paired-associate lists. Journal of Experimental Psychology: Learning, Memory, and Cognition, 6(1), 91-105.

Rotello, C. M., Masson, M. E. J., \& Verde, M. F. (2008). Type I error rates and power analyses for single-point sensitivity measures. Perception $\&$ Psychophysics, $70(2)$, $389-401$.

Rouder, J. N., \& Lu, J. (2005). An introduction to Bayesian hierarchical models with an application in the theory of signal detection. Psychonomic Bulletin \& Review, 12(4), $573-604$.

Rumelhart, D. E. (1990). Brain style computation: Learning and generalization. In S. F. Zornetzer, J. L. Davis, \& C. Lau (Eds.), An introduction to neural and electronic networks (pp. 405-420). Academic Press.

Sahakyan, L., \& Hendricks, H. E. (2012). Context change and retrieval difficulty in the list-before-last paradigm. Memory \& Cognition, 40(6), 844-860.

Schölkopf, B., \& Smola, A. J. (2002). Learning with Kernels. MIT Press.

Schulman, A. L. (1974). The declining course of recognition memory. Memory and Cogni- 
tion, 2, 14-18.

Sederberg, P. B., Howard, M. W., \& Kahana, M. J. (2008). A context-based theory of recency and contiguity in free recall. Psychological Review, 115(4), 893-912.

Shepard, R. N. (1967). Recognition memory for words, sentences, and pictures. Journal of Verbal Learning and Verbal Behavior, 6, 156-163.

Shiffrin, R. M., Huber, D. E., \& Marinelli, K. (1995). Effects of category length and strength on familiarity in recognition. Journal of Experimental Psychology: Learning, Memory, and Cognition, 21(2), 267-287.

Shiffrin, R. M., Lee, M. D., Kim, W., \& Wagenmakers, E. J. (2008). A survey of model evaluation approaches with a tutorial on hierarchical Bayesian methods. Cognitive Science, 32, 1248-1284.

Shiffrin, R. M., Ratcliff, R., \& Clark, S. E. (1990). List-strength effect: II. Theoretical mechanisms. Journal of Experimental Psychology: Learning Memory and Cognition, $16(2), 179-195$.

Shiffrin, R. M., \& Steyvers, M. (1997). A model for recognition memory: REM - retrieving effectively from memory. Psychonomic Bulletin $\&$ Review, 4(2), 145-166.

Singer, M. (2009). Strength-based criterion shifts in recognition memory. Memory $\&$ Cognition, 37(7), 976-984.

Standing, L. (1973). Learning 10,000 pictures. Quarterly Journal of Experimental Psychology, 25(2).

Starns, J. J., \& Ratcliff, R. (2014). Validating the unequal-variance assumption in recognition memory using response time distributions instead of ROC functions: A diffusion model analysis. Journal of Memory and Language, 70, 36-52.

Starns, J. J., Ratcliff, R., \& McKoon, G. (2012). Evaluating the unequal-variance and dualprocess explanations of the zROC slopes with response time data and the diffusion model. Cognitive Psychology, 64, 1-34.

Starns, J. J., Ratcliff, R., \& White, C. N. (2012). Diffusion model drift rates can be influenced by decision processes: An analysis of the strength-based mirror effect. Journal of Experimental Psychology: Learning, Memory, and Cognition, 38(5), 1137- 
1151.

Starns, J. J., Rotello, C. M., \& Ratcliff, R. (2012). Mixing strong and weak targets provides no evidence against the unequal-variance explanation of zROC slope: A comment on Koen and Yonelinas (2010). Journal of Experimental Psychology: Learning, Memory, and Cognition, 38(3), 793-801.

Starns, J. J., White, C. N., \& Ratcliff, R. (2010). A direct test of the differentiation mechanism: REM, BCDMEM, and the strength-based mirror effect in recognition memory. Journal of Memory and Language, 63, 18-34.

Starns, J. J., White, C. N., \& Ratcliff, R. (2012). The strength-based mirror effect in subjective strength ratings: The evidence for differentiation can be produced without differentiation. Memory \& Cognition, 40(8), 1189-1199.

Stretch, V., \& Wixted, J. T. (1998a). Decision rules for recognition memory confidence judgments. Journal of Experimental Psychology: Learning, Memory, and Cognition, $24(6), 1397-1410$.

Stretch, V., \& Wixted, J. T. (1998b). On the differences between strength-based and frequency-based mirror effects in recognition memory. Journal of Experimental Psychology: Learning, Memory, and Cognition, 24(6), 1379-1396.

Strong, E. K. J. (1912). The effect of length of series upon recognition memory. Psychological Review, 19, 447-462.

Tehan, G., \& Humphreys, M. S. (1995). Transient phonemic codes and immunity to proactive interference. Memory \&6 Cognition, 23(2), 181-191.

Tehan, G., \& Humphreys, M. S. (1996). Cuing effects in short-term recall. Memory \& Cognition, 24(6), 719-732.

Treves, A., \& Rolls, E. T. (1992). Computational constraints suggest the need for two distinct input systems to the hippocampal CA3 network. Hippocampus, 2(2), 189200.

Tulving, E., \& Hastie, R. (1972). Inhibition Effects of Intralist Repetition in Free Recall. Journal of Experimental Psychology, 92(3), 297-304.

Tulving, E., \& Thomson, D. M. (1973). Encoding specificity and retrieval processes in 
episodic memory. Psychological Review, 80, 352-373.

Turner, B. M., Dennis, S., \& Van Zandt, T. (2013). Likelihood-free Bayesian analysis of memory models. Psychological Review, 120(3), 667-678.

Underwood, B. J. (1978). Recognition memory as a function of the length of study list. Bulletin of the Psychonomic Society, 12, 89-91.

Usher, M., \& McClelland, J. L. (2001). The time course of perceptual choice: The leaky, competing accumulator model. Psychological Review, 108, 550-592.

Vandekerckhove, J., Tuerlinckx, F., \& Lee, M. D. (2011). Hierarchical diffusion models for two-choice response times. Psychological Methods, 16(1), 44-62.

Vanpaemel, W., \& Lee, M. D. (2012). Using priors to formalize theory: Optimal attention and the generalized context model. Psychonomic Bulletin \& Review, 19(6), 10471056.

Ward, G. (2002). A recency-based account of the list length effect in free recall. Memory E Cognition, 30(6), 885-892.

Watkins, O. C., \& Watkins, M. J. (1975). Buildup of proactive inhibition as a cue-overload effect. Journal of Experimental Psychology: Human Learning and Memory, 104(4), $442-452$.

Wickelgren, W. A. (1968). Unidimensional strength theory and component analysis of noise in absolute and comparative judgments. Journal of Mathematical Psychology, $5,102-122$.

Wickelgren, W. A., \& Norman, D. A. (1966). Strength models and serial position in short-term recognition memory. Journal of Mathematical Psychology, 3, 316-347.

Wickens, D. D. (1970). Encoding categories of words: An empirical approach to meaning. Psychological Review, 77(1), 1-15.

Wickens, D. D., Born, D. G., \& Allen, C. K. (1963). Proactive inhibition and item similarity in short-term memory. Journal of Verbal Learning and Verbal Behavior, 2, 440-445.

Wickens, T. D. (2002). Elementary signal detection theory. Oxford University Press.

Wixted, J. T. (1992). Subjective memorability and the mirror effect. Journal of Experimental Psychology: Learning, Memory, and Cognition, 18(4), 681-690. 
Wixted, J. T. (2007). Dual-process theory and signal-detection theory of recognition memory. Psychological Review, 114(1), 152-176.

Xu, J., \& Malmberg, K. J. (2007). Modeling the effects of verbal and nonverbal pair strength on associative recognition. Memory \& Cognition, 35(3), 526-544.

Yonelinas, A. P. (1994). Receiver-operating characteristics in recognition memory: Evidence for a dual-process model. Journal of Experimental Psychology: Learning, Memory, and Cognition, 20(6), 1341-1354.

Yonelinas, A. P., Hockley, W. E., \& Murdock, B. B. (1992). Tests of the List-Strength Effect in Recognition Memory. Journal of Experimental Psychology: Learning, Memory, and Cognition, 18(2), 345-355.

Zaki, S. R., \& Nosofsky, R. M. (2001). Exemplar accounts of blending and distinctiveness effects in perceptual old new recognition. Journal of Experimental Psychology: Learning, Memory, and Cognition, 27, 1022-1041.

Zechmeister, E. B., Curt, C., \& Sebastian, J. A. (1978). Errors in a recognition memory task are a U-shaped function of word frequency. Bulletin of the Psychonomic Society, $11,371-373$. 


\section{Appendix A \\ DERIVATIONS FOR THE MEAN AND VARiance of MEMORY Strength in Associative ReCOGNition}

In associative recognition, the co-occurrence tensor $M_{c}$ can be decomposed into the memories that are contributing to the memory strength $s$ that is generated at retrieval from a list of pairs $L$. Pairs stored during the list episode are scaled by the associative learning rate $r_{\text {assoc }}$. Given that I am investigating datasets in which the word pairs are random pairings of unrelated words, it is assumed that the probability of having seen a pair combination prior to the experiment is negligible and thus the context noise term can be omitted. I also assume no common words among the word pairs:

$$
\begin{array}{cr}
s_{\text {intact }}=\left(C_{s}^{\prime} \otimes I_{a}^{\prime} \otimes I_{b}^{\prime}\right) \cdot r_{\text {assoc }}\left(C_{s} \otimes I_{a} \otimes I_{b}\right)+ & \text { Self Match } \\
\sum_{i, j \in L, i, j \neq a, b} r_{a s s o c}\left(C_{s} \otimes I_{i} \otimes I_{j}\right)+ & \text { Item Noise } \\
\sum_{u \in P, u \neq s}\left(C_{u} \otimes I_{a} \otimes I_{b}\right) & \text { Background Noise }
\end{array}
$$

If the probe cue is a rearranged pair, there is no self match. However, there are two partial matches terms. For a list of pairs A-B, C-D, E-F, etc., a rearranged pair A-D will have a partial match to the stored A-B and C-D pairs: 


$$
\begin{aligned}
& s_{\text {rearranged }}=\left(C_{s}^{\prime} \otimes I_{a}^{\prime} \otimes I_{d}^{\prime}\right) \cdot r_{\text {assoc }}\left(C_{s} \otimes I_{a} \otimes I_{b}\right) \quad \text { Partial Match } \\
& +r_{\text {assoc }}\left(C_{s} \otimes I_{c} \otimes I_{d}\right) \quad \text { Partial Match } \\
& +\sum_{i, j \in L, i, j \neq a, b, c, d} r_{a s s o c}\left(C_{s} \otimes I_{i} \otimes I_{j}\right) \quad \text { Item Noise } \\
& +\sum_{u \in P, u \neq s}\left(C_{u} \otimes I_{y} \otimes I_{z}\right) \quad \text { Background Noise }
\end{aligned}
$$

The above equations can be rewritten as matches and mismatches between the item and context vectors:

$$
\begin{aligned}
& s_{\text {intact }}=r_{\text {assoc }}\left(C_{s}^{\prime} \cdot C_{s}\right)\left(I_{a}^{\prime} \cdot I_{a}\right)\left(I_{b}^{\prime} \cdot I_{b}\right)+\quad \text { Self Match } \\
& \sum_{i, j \in L, i, j \neq a, b} r_{a s s o c}\left(C_{s}^{\prime} \cdot C_{s}\right)\left(I_{a}^{\prime} \cdot I_{i}\right)\left(I_{b}^{\prime} \cdot I_{j}\right)+\quad \text { Item Noise } \\
& \sum_{u \in P, u \neq s}\left(C_{s}^{\prime} \cdot C_{u}\right)\left(I_{a}^{\prime} \cdot I_{y}\right)\left(I_{a}^{\prime} \cdot I_{z}\right) \quad \text { Background Noise } \\
& s_{\text {rearranged }}=r_{\text {assoc }}\left(C_{s}^{\prime} \cdot C_{s}\right)\left(I_{a}^{\prime} \cdot I_{a}\right)\left(I_{d}^{\prime} \cdot I_{b}\right)+\quad \text { Partial Match } \\
& r_{\text {assoc }}\left(C_{s}^{\prime} \cdot C_{s}\right)\left(I_{a}^{\prime} \cdot I_{c}\right)\left(I_{d}^{\prime} \cdot I_{d}\right)+\quad \text { Partial Match } \\
& \sum_{i, j \in L, i, j \neq a, b, c, d} r_{a s s o c}\left(C_{s}^{\prime} \cdot C_{s}\right)\left(I_{a}^{\prime} \cdot I_{i}\right)\left(I_{b}^{\prime} \cdot I_{j}\right)+\quad \text { Item Noise } \\
& \sum_{u \in P, u \neq s}\left(C_{s}^{\prime} \cdot C_{u}\right)\left(I_{a}^{\prime} \cdot I_{y}\right)\left(I_{a}^{\prime} \cdot I_{z}\right) \quad \text { Background Noise }
\end{aligned}
$$

The matches and mismatches of the context vectors can be substituted using the same parameters for the normal distribution used in Equation 2.7 for item recognition. 


\section{Appendix B EQUATIONS FOR THE LOG LiKELIHOOD RATIO TRANSFORMATION of Memory Strength}

For this model, the analytic expressions for the likelihood ratio transformation derived by Glanzer et al. (2009) for both the equal variance and unequal variance normal distributions. In this model, the ratio of standard deviations $\sigma_{n e w} / \sigma_{\text {old }}$ depends on a number of model parameters, and ratios can be one or greater although ratios below one are the most common. Following Glanzer et al., I use $X$ to refer to samples on the memory strength axis and $\Lambda$ to refer to log likelihood ratios.

The equations described in Glanzer et al. (2009) describe a fully informed likelihood ratio model where the actual memory strengths are equivalent to the strengths used in the likelihood ratio. As described in the text, the expected strengths in the likelihood ratio need not be equivalent to the actual strengths. In the mixed lists in the list strength datasets, I assume that despite the fact that tested items have different strengths, the same expected strengths in the likelihood ratio are used for each item. I describe the expected strengths as "subjective strengths." I describe the actual means and standard deviations of the memory strength distributions as $\mu$ and $\sigma$, while the subjective means and standard deviations of the memory strength distributions are denoted as $\rho$ and $\tau$.

Glanzer et al.'s equations were written expressed for the case where $\sigma_{n e w}=1$ and differences in strength were expressed as different values of $\mu_{\text {old }}$. For that reason, in their expressions $d^{\prime}$ and $d$ equal $\mu_{\text {old }}$ in the unequal variance and equal variance models, respec- 
tively. Given that in this model both $\sigma_{\text {new }}$ and $\sigma_{\text {old }}$ vary, all parameters are normalized by $\tau_{\text {new }}$ before usage in any of the equations below.

For the equal variance log likelihood ratio transformation, I use the equations in Appendix A of Glanzer et al. (2009). This results in normal distributions with means and variances as follows:

$$
\begin{aligned}
E(\Lambda \mid \text { Old }) & =d^{\prime 2} / 2 \\
E(\Lambda \mid N e w) & =d^{\prime} \mu_{\text {old }}-d^{\prime 2} / 2 \\
\operatorname{Var}(\Lambda) & =d^{\prime 2} \sigma_{\text {old }}^{2}
\end{aligned}
$$

where $d^{\prime}=\rho_{\text {old }} / \tau_{\text {new }} . \mu_{\text {old }}$ and $\sigma_{\text {old }}^{2}$ are normalized by $\tau_{\text {new }}$ and $\tau_{\text {new }}^{2}$, respectively.

For the unequal variance log likelihood ratio transformation, I use the equations in Appendix B of Glanzer et al. (2009). Like with the equal variance case, $\sigma_{\text {new }}$ is assumed to be one. The parameter $\varsigma$ refers to the subjective ratio of standard deviations $\tau_{\text {old }} / \tau_{\text {new }}$. The resulting log likelihood ratio distributions are non-central chisquare distributions, where the $x$ is:

$$
\frac{\Phi+\left(d^{2}\right) /\left[2 \varsigma^{2}\left(\varsigma^{2}-1\right)\right]-\left(d^{2} / 2 \varsigma^{2}\right)-\log (\varsigma)}{\sigma_{X}^{2}\left(\varsigma^{2}-1\right) / 2 \varsigma^{2}}
$$

where $\Phi$ is the criterion on the $\log$ likelihood ratio axis and $d=\rho_{\text {old }} / \tau_{\text {new }}, \sigma_{X}^{2}$ is the actual variance of the target or lure distribution normalized by $\tau_{n e w}^{2}$. The noncentral chisquare distribution has one degree of freedom and non-centrality:

$$
\left(\frac{-d /\left(\varsigma^{2} / 2\right)-\mu_{X}}{\sigma_{X}}\right)^{2}
$$

where $\mu_{X}$ and $\sigma_{X}$ are the actual means and standard deviations of the target or lure distribution normalized by $\tau_{\text {new }}$. Predictions for old or new items can be derived from these equations by substituting values of the appropriate distribution for $\mu_{X}$ and $\sigma_{X}$. 
One should note that it is also possible for this model to produce cases where $\sigma_{\text {new }}>\sigma_{\text {old }}$ (although in the model fits, it was quite rare). Hit and false alarm rate predictions are produced for this case by merely taking using the cumulative distribution function with the parameters above, while in the standard $\sigma_{\text {old }}>\sigma_{\text {new }}$ case, hit and false alarm rates are produced by subtracting the cumulative distribution function from one. 


\section{Appendix $\mathrm{C}$ \\ Binomial and Multinomial Rate EsTIMATION}

While some investigations estimate the relevant parameters from signal detection theory $\left(d^{\prime}\right.$ and c) and derive the rates directly from those estimates (Dennis et al., 2008; Pooley et al., 2011), I did not want the rates to have to conform to a particular signal detection model. Instead, rates were estimated directly from the hit and false alarm counts.

All rates for individual participants were sampled from reparameterized beta distributions, which uses a mean parameter $\lambda$ and variance parameter $\nu$. Separate $\lambda$ and $\nu$ parameters were used for each hit and false alarm rate for each condition. The hyperparameters for the means and variances used non-informative priors:

$$
\begin{aligned}
& \lambda \sim \operatorname{Beta}(.5,2) \\
& \nu \sim \operatorname{InverseGamma}(.1, .1)
\end{aligned}
$$

For the confidence counts in the DeCarlo (2007) dataset, multinomial rates for each confidence category were sampled from reparameterized beta distributions, but were subsequently normalized to sum to one.

Each rate parameter was estimated with four chains of 5,000 samples each after a burn-in period of 1,500 samples using JAGS software. 